\title{
DOUBLY CONNECTED V-STATES FOR THE GENERALIZED SURFACE QUASI-GEOSTROPHIC EQUATIONS
}

\author{
FRANCISCO DE LA HOZ, ZINEB HASSAINIA, AND TAOUFIK HMIDI
}

\begin{abstract}
In this paper, we prove the existence of doubly connected V-states for the generalized SQG equations with $\alpha \in] 0,1[$. They can be described by countable branches bifurcating from the annulus at some explicit "eigenvalues" related to Bessel functions of the first kind. Contrary to Euler equations [23], we find V-states rotating with positive and negative angular velocities. At the end of the paper we discuss some numerical experiments concerning the limiting V-states.
\end{abstract}

\section{Contents}

1. Introduction 1

2. Boundary equations 8

3. Tools 10

3.1. Functional spaces 10

3.2. Elements of the bifurcation theory 11

3.3. Special functions 11

3.4. Basic integrals 13

4. Regularity of the nonlinear functional 18

5. Spectral study 27

5.1. Linearized operator 28

5.2. Asymptotic behavior 34

5.3. Monotonicity of the eigenvalues 37

6. Bifurcation at simple eigenvalues 40

7. Numerical study of $V$-states 48

7.1. Simply-connected $V$-states 48

7.2. Doubly-connected $V$-states $\quad 56$

References $\quad 64$

\section{INTRODUCTION}

The present work deals with the generalized surface quasi-geostrophic equation (gSQG) arising in fluid dynamics and which describes the evolution of the potential temperature $\theta$ by the transport equation:

2000 Mathematics Subject Classification. 35Q35, 76B03, 76C05.

Key words and phrases. gSQG equations, V-states, doubly connected patches, bifurcation theory. 


$$
\left\{\begin{array}{l}
\partial_{t} \theta+u \cdot \nabla \theta=0, \quad(t, x) \in \mathbb{R}_{+} \times \mathbb{R}^{2}, \\
u=-\nabla^{\perp}(-\Delta)^{-1+\frac{\alpha}{2}} \theta, \\
\theta_{\mid t=0}=\theta_{0} .
\end{array}\right.
$$

Here $u$ refers to the velocity field, $\nabla^{\perp}=\left(-\partial_{2}, \partial_{1}\right)$ and $\alpha$ is a real parameter taken in ]0,2[. The singular operator $(-\Delta)^{-1+\frac{\alpha}{2}}$ is of convolution type and defined by,

$$
(-\Delta)^{-1+\frac{\alpha}{2}} \theta(x)=\frac{C_{\alpha}}{2 \pi} \int_{\mathbb{R}^{2}} \frac{\theta(y)}{|x-y|^{\alpha}} d y,
$$

with $C_{\alpha}=\frac{\Gamma(\alpha / 2)}{2^{1-\alpha} \Gamma\left(\frac{2-\alpha}{2}\right)}$ where $\Gamma$ stands for the gamma function. This model was proposed by Córdoba et al. in [14] as an interpolation between Euler equations and the surface quasi-geostrophic model (SQG) corresponding to $\alpha=0$ and $\alpha=1$, respectively. The SQG equation was used by Juckes [26] and Held et al. [21] to describe the atmosphere circulation near the tropopause. It was also used by Lapeyre and Klein [31] to track the ocean dynamics in the upper layers. We note that there is a strong mathematical and physical analogy with the three-dimensional incompressible Euler equations; see [13] for details.

In the last few years there has been a growing interest in the mathematical study of these active scalar equations. Special attention has been paid to the local well-posedness of classical solutions which can be performed in various functional spaces. For instance, this was implemented in the framework of Sobolev spaces [10] by using the commutator theory. Wether or not these solutions are global in time is an open problem except for Euler equations $\alpha=0$. The second restriction with the gSQG equation concerns the construction of Yudovich solutions - known to exist globally in time for Euler equations [46] - which are not at all clear even locally in time. The main difficulty is due to the velocity which is in general singular and scales below the Lipschitz class. Nonetheless one can say more about this issue for some special class of concentrated vortices. More precisely, when the initial datum has a vortex patch structure, that is, $\theta_{0}(x)=\chi_{D}$ is the characteristic function of a bounded simply connected smooth domain $D$, then there is a unique local solution in the patch form $\theta(t)=\chi_{D_{t}}$. In this case, the boundary motion of the domain $D_{t}$ is described by the contour dynamics formulation; see the papers $[19,40]$. The global persistence of the boundary regularity is only known for $\alpha=0$ according to Chemin's result [12]; for another proof see the paper of Bertozzi and Constantin [4]. Notice that for $\alpha>0$ the numerical experiments carried out in [14] provide strong evidence for the singularity formation in finite time. Let us mention that the contour dynamics equation remains locally well-posed when the domain of the initial patch is assumed to be multi-connected meaning that the boundary is composed with finite number of disjoint smooth Jordan curves.

The main concern of this work is to explore analytically and numerically some special vortex patches called V-states; they correspond to patches which do not change their shapes during the motion. The emphasis will be put on the V-states subject to uniform rotation around their center of mass, that is, $D_{t}=\mathbf{R}_{x_{0}, \Omega t} D$, where $\mathbf{R}_{x_{0}, \Omega t}$ stands for the planar rotation with center $x_{0}$ and angle $\Omega t$. The parameter $\Omega$ is called the angular velocity of the rotating domain. Along the chapter we call these structures rotating patches or simply V-states. Their existence is of great interest for at least two reasons: first they provide non trivial initial data with global existence, and second this might explain the emergence of some ordered structures in the geophysical flows. This study has been conducted first for the 
two-dimensional Euler equations $(\alpha=0)$ a long time ago and a number of analytical and numerical studies are known in the literature. The first result in this setting goes back to Kirchhoff [29] who discovered that an ellipse of semi-axes $a$ and $b$ rotates uniformly with the angular velocity $\Omega=a b /(a+b)^{2}$; see for instance the references [5, p. 304] and [30, p. 232]. Till now this is the only known explicit V-states; however the existence of implicit examples was established about one century later. In fact, Deem and Zabusky [16] gave numerical evidence of the existence of the $\mathrm{V}$-states with $m$-fold symmetry for each integer $m \geq 2$; remark that the case $m=2$ coincides with Kirchhoff's ellipses. To fix the terminology, a planar domain is said $m$-fold symmetric if it has the same group invariance of a regular polygon with $m$ sides. Note that at each frequency $m$ these V-states can be seen as a continuous deformation of the disc with respect to to the angular velocity. An analytical proof of this fact was given few years later by Burbea in [6]. His approach consists in writing a stationary problem in the frame of the patch with the conformal mapping of the domain and to look for the non trivial solutions by using the technique of the bifurcation theory. Quite recently, in [24] Burbea's approach was revisited with more details and explanations. The boundary regularity of the V-states was also studied and it was shown to be of class $C^{\infty}$ and convex close to the disc.

We mention that explicit vortex solutions similar to the ellipses are discovered in the literature for the incompressible Euler equations in the presence of an external shear flow; see for instance $[11,27,35]$. A general review about vortex dynamics can be found in the papers $[3,36]$.

With regard to the existence of the simply connected V-states for the (gSQG) it has been discussed very recently in the papers $[8,7,20]$. In [7], it was shown that the ellipses cannot rotate for any $\alpha \in(0,2)$ and to the authors' best knowledge no explicit example is known in the literature. Lately, in [20] the last two authors proved the analogous of Burbea's result and showed the existence of the $m$-folds rotating patches for $\alpha \in] 0,1[$. In addition, the bifurcation from the unit disc occurs at the angular velocities,

$$
\Omega_{m}^{\alpha} \triangleq \frac{\Gamma(1-\alpha)}{2^{1-\alpha} \Gamma^{2}\left(1-\frac{\alpha}{2}\right)}\left(\frac{\Gamma\left(1+\frac{\alpha}{2}\right)}{\Gamma\left(2-\frac{\alpha}{2}\right)}-\frac{\Gamma\left(m+\frac{\alpha}{2}\right)}{\Gamma\left(m+1-\frac{\alpha}{2}\right)}\right), \quad m \geq 2,
$$

where $\Gamma$ denotes the usual gamma function. The remaining case $\alpha \in[1,2)$ has been explored and solved by Castro, Córdoba and Gómez-Serrano in [8]. They also show that the V-states are $C^{\infty}$ and convex close to the discs. To complete these works we discussed in this work ( which is forwarded in Section ??) some numerical experiments concerning these V-states and their limiting structures when we go to the end of each branch; new behaviors will be observed compared to the numerical experiments achieved for Euler case [42].

We want in this chapter to learn more about the V-states but with different topological structure compared to the preceding discussion. More precisely, we propose to scrutinize rotating patches with only one hole, also called doubly connected V-states. Recall that a patch $\theta_{0}=\chi_{D}$ is said to be doubly connected if the domain $D=D_{1} \backslash D_{2}$, with $D_{1}$ and $D_{2}$ being two simply connected bounded domains satisfying $\overline{D_{2}} \subset D_{1}$. This structure is preserved for Euler system globally in time but known to be for short time when $\alpha \in] 0,1[$ see $[10,19,40]$. We notice that compared to the simply connected case the boundaries evolve through extra nonlinear terms coming from the interaction between the boundaries and therefore the existence of the $\mathrm{V}$-states is relatively more complicate to analyze. This problem is not well studied from the analytical point of view and recent progress has been 
made for Euler equations in the papers [18, 25, 23]. In [25], the authers proved the existence of explicit V-states similar to Kirchhoff ellipses seems to be out of reach. Indeed, it was stated that if one of the boundaries of the $\mathrm{V}$-state is a circle then necessarily the other one should be also a circle. Moreover, if the inner curve is an ellipse then there is no rotation at all. Another closely related subject is to deal with some vortex magnitude $\mu$ inside the domain $D_{2}$ and try to find explicit rotating patches. This was done by Flierl and Polvani [18] who proved that confocal ellipses rotate uniformly provided some compatibility relations are satisfied between the parameter $\mu$ and the semi-axes of the ellipses. We note that another approach based upon complex analysis tools with a complete discussion can be found in [25]. Now, from the equations (1.1) we may easily conclude that the annulus is a stationary doubly connected patch, and therefore it rotates with any angular velocity $\Omega$. From this obvious fact, one can wonder wether or not the bifurcation to nontrivial $\mathrm{V}$-states still happens as for the simply connected case. This has been recently investigated in [23] for Euler equations following basically Burbea's approach but with more involved calculations. It was shown that for $b \in(0,1)$ and $m$ being an integer satisfying the inequality

$$
1+b^{m}-\frac{1-b^{2}}{2} m<0
$$

then there exist two curves of non-annular $m$-fold doubly connected patches bifurcating from the annulus $\{z ; b<|z|<1\}$ at different eigenvalues $\Omega_{m}^{ \pm}$given explicitly by the formula

$$
\Omega_{m}^{ \pm}=\frac{1-b^{2}}{4} \pm \frac{1}{2 m} \sqrt{\left[\frac{m}{2}\left(1-b^{2}\right)-1\right]^{2}-b^{2 m}} .
$$

Now we come to the main contribution of the current work. We propose to study the doubly connected V-states for the gSQG model (1.1) when $\alpha \in] 0,1[$. Before stating our result we need to make some notation. We define

$$
\Lambda_{n}(b) \triangleq \frac{1}{b} \int_{0}^{+\infty} J_{n}(b t) J_{n}(t) \frac{d t}{t^{1-\alpha}}
$$

and

$$
\Theta_{n} \triangleq \Lambda_{1}(1)-\Lambda_{n}(1)
$$

where $J_{n}$ refers to Bessel function of the first kind. Our result reads as follows.

Theorem 1.1. Let $\alpha \in[0,1[$ and $b \in] 0,1[$; there exists $N \in \mathbb{N}$ with the following property: For each $m>N$ there exists two curves of $m$-fold doubly connected $V$-states that bifurcate from the annulus $\{z \in \mathbb{C}, b<|z|<1\}$ at the angular velocities

$$
\Omega_{m}^{\alpha, \pm} \triangleq \frac{1-b^{2}}{2} \Lambda_{1}(b)+\frac{1}{2}\left(1-b^{-\alpha}\right) \Theta_{m} \pm \frac{1}{2} \sqrt{\Delta_{m}(\alpha, b)}
$$

with

$$
\Delta_{m}(\alpha, b) \triangleq\left[\left(b^{-\alpha}+1\right) \Theta_{m}-\left(1+b^{2}\right) \Lambda_{1}(b)\right]^{2}-4 b^{2} \Lambda_{m}^{2}(b) .
$$

Remarks. (1) The number $N$ is the smallest integer such that

$$
\Theta_{N} \geq \frac{1+b^{2}}{b^{-\alpha}+1} \Lambda_{1}(b)+\frac{2 b}{b^{-\alpha}+1} \Lambda_{N}(b) .
$$


This restriction appears in the spectral study of the linearized operator and gives only sufficient condition for the existence of the $V$-states.

(2) As we shall see later in Lemma 5.2, for $\alpha=0$ we find the result of Euler equations established in [23] and the condition (1.4) is in accordance with that given by (1.3).

(3) We can check by using the strict monotonicity of $b \mapsto \Lambda_{1}(b)$ that for any $b, \alpha \in(0,1)$,

$$
\lim _{m \rightarrow+\infty} \Omega_{m}^{\alpha,-}=-b^{-\alpha} \Lambda_{1}(1)+\Lambda_{1}(b)<0 .
$$

Consequently the corresponding bifurcating curves generate close to the annulus non trivial clockwise doubly connected $V$-states. This fact is completely new compared to what we know for Euler equations or for the simply connected case where the bifurcation occurs at positive angular velocities. The numerical experiments discussed in Section 7 reveal the existence of non radial stationary patches for the generalized quasi-geostrophic equations and it would be very interesting to establish this fact analytically. In a connected subject, we point out that the last author has shown quite recently in [22] that for Euler equations clockwise convex V-states reduce to the discs.

Now we shall sketch the proof of Theorem 1.1 which is mainly based upon the bifurcation theory via Crandall-Rabinowitz's Theorem. The first step is to write down the analytical equations of the boundaries of the V-states. This can be done for example through the conformal parametrization of the domains $D_{1}$ and $D_{2}$ : we denote by $\phi_{j}: \mathbb{D}^{c} \rightarrow D_{j}^{c}$ the conformal mappings possessing the following structure,

$$
\forall|w| \geq 1, \quad \phi_{1}(w)=w+\sum_{n \in \mathbb{N}} \frac{a_{n}}{w^{n}}, \quad \phi_{2}(w)=b w+\sum_{n \in \mathbb{N}} \frac{c_{n}}{w^{n}} .
$$

We assume in addition that the Fourier coefficients are real which means that we look only for the V-states which are symmetric with respect to the real axis. Moreover using the subordination principle we deduce that $b \in] 0,1[$; the parameter $b$ coincides with the small radius of the annulus that we slightly perturb. As we shall see later in Section 2, the conformal mappings are subject to two coupled nonlinear equations defined as follows: for $j \in\{1,2\}$

$$
\begin{aligned}
F_{j}\left(\Omega, \phi_{1}, \phi_{2}\right)(w) & \triangleq \operatorname{Im}\left\{\left(\Omega \phi_{j}(w)+S\left(\phi_{2}, \phi_{j}\right)(w)-S\left(\phi_{1}, \phi_{j}\right)(w)\right) \bar{w} \overline{\phi_{j}^{\prime}(w)}\right\} \\
& =0, \quad \forall w \in \mathbb{T}
\end{aligned}
$$

with

$$
S\left(\phi_{i}, \phi_{j}\right)(w)=C_{\alpha} f_{\mathbb{T}} \frac{\phi_{i}^{\prime}(\tau)}{\left|\phi_{j}(w)-\phi_{i}(\tau)\right|^{\alpha}} d \tau \quad \text { and } \quad C_{\alpha} \triangleq \frac{\Gamma(\alpha / 2)}{2^{1-\alpha} \Gamma\left(\frac{2-\alpha}{2}\right)} .
$$

In order to apply the bifurcation theory we should understand the structure of the linearized operator around the trivial solution $\left(\phi_{1}, \phi_{2}\right)=(\mathrm{Id}, b \mathrm{Id})$, corresponding to the annulus with radii $b$ and 1 , and identify the range of $\Omega$ where this operator has a one-dimensional kernel. The computations of the linear operator $D F(\Omega, \mathrm{Id}, b \mathrm{Id})$ with $F=\left(F_{1}, F_{2}\right)$ in terms of its Fourier coefficients are long and tricky. They are connected to the hypergeometric functions ${ }_{2} F_{1}(a, b ; c ; z)$ simply denoted by $F(a, b ; c ; z)$ throughout this chapter. To find compact formula we use at several steps some algebraic identities described by the contiguous function 
relations (3.11)-(3.17). Similarly to the Euler equations [23] the linearized operator acts as a matrix Fourier multiplier. More precisely, for

$$
h_{1}(w)=\sum_{n \geq 1} \frac{a_{n}}{w^{n}}, \quad h_{2}(w)=\sum_{n \geq 1} \frac{c_{n}}{w^{n}},
$$

we obtain the formula,

$$
D F(\Omega, \operatorname{Id}, b \operatorname{Id})\left(h_{1}, h_{2}\right)(w)=\frac{i}{2} \sum_{n \geq 1}(n+1) M_{n+1}^{\alpha}\left(\begin{array}{c}
a_{n} \\
c_{n}
\end{array}\right)\left(w^{n+1}-\bar{w}^{n+1}\right),
$$

where the matrix $M_{n}$ is given for $n \geq 2$ by

$$
M_{n}^{\alpha} \triangleq\left(\begin{array}{cc}
\Omega-\Theta_{n}+b^{2} \Lambda_{1}(b) & -b^{2} \Lambda_{n}(b) \\
b \Lambda_{n}(b) & b \Omega+b^{1-\alpha} \Theta_{n}-b \Lambda_{1}(b)
\end{array}\right) .
$$

Therefore the values of $\Omega$ associated to non trivial kernels are the solutions of a second-degree polynomial,

$$
\operatorname{det} M_{n}^{\alpha}=0 .
$$

This can be solved when the discriminant $\Delta_{n}(\alpha, b)$ introduced in Theorem 1.1 is positive. The computations of the dimension of the kernel are more complicate than the cases raised before in the references $[20,23]$. The matter reduces to count the following discrete set

$$
\left\{n \geq 2, \operatorname{det} M_{n}^{\alpha}=0\right\} .
$$

Note that in $[20,23]$ this set has only one element and therefore the kernel is one-dimensional. This follows from the monotonicity of the "nonlinear eigenvalues" sequence $n \mapsto \Omega$ which is not very hard to get due to the explicit polynomial structure of the coefficients of the analogous polynomial to (1.5). Unfortunately, in the current situation this structure is broken because the matrix coefficients of $M_{n}^{\alpha}$ are related to Bessel functions. Therefore the monotonicity of the eigenvalues is more subtle and will require more refined analysis. This subject will be discussed later with ample details in the Subsection 5.3. To achieve the spectral study and check the complete assumptions of Crandall-Rabinowitz's Theorem it remains to prove the transversality assumption and check that the image is of co-dimension one. This will be done in Section 6 in a straightforward way and without serious difficulties. We also mention that the transversality assumption is obtained only when the discriminant $\Delta_{n}(\alpha, b)>0$ meaning that there is no crossing roots for the equation (1.5). The proof of the bifurcation will be achieved in Section 6. Next, we shall make few comments about the statement of the main theorem.

Remarks. 1) For the SQG equation corresponding to $\alpha=1$ the situation is more delicate due to some logarithmic loss. The simply connected case has been achieved recently in [8] by using Hilbert spaces where we take into account this loss. The same approach could lead to the existence of the doubly-connected V-states for the (SQG) equation. For the spectral study, the linearized operator can be obtained as a limit of (1.5) when a goes to 1. More precisely, we get

$$
D F(\Omega, I d, b I d)\left(h_{1}, h_{2}\right)(w)=\frac{i}{2} \sum_{n \geq 1}(n+1) M_{n+1}^{1}\left(\begin{array}{c}
a_{n} \\
c_{n}
\end{array}\right)\left(w^{n+1}-\bar{w}^{n+1}\right),
$$


where the matrix $M_{n}$ is given for $n \geq 2$ by

$$
M_{n}^{1} \triangleq\left(\begin{array}{cc}
\Omega-\Theta_{n}+b^{2} \Lambda_{1}(b) & -b^{2} \Lambda_{n}(b) \\
b \Lambda_{n}(b) & b \Omega+\Theta_{n}-b \Lambda_{1}(b)
\end{array}\right),
$$

with

$$
\Theta_{n}=\frac{2}{\pi} \sum_{k=1}^{n-1} \frac{1}{2 k+1} \quad \text { and } \quad \Lambda_{n}(b)=\frac{1}{b} \int_{0}^{+\infty} J_{n}(b t) J_{n}(t) d t
$$

3) The boundary of the $V$-states belongs to Hölder space $C^{2-\alpha}$. For Euler equations corresponding to $\alpha=0$, we get better result in the simply connected geometry as it was shown in [24]; the boundary is $C^{\infty}$ and convex when the $V$-states are close to the circle. The proof in this particular case uses in a deep way the algebraic structure of the kernel according to some recurrence formulae. The extension of this result to $\alpha \in] 0,2[$ was done in [8]. We expect that the latter approach could be also adapted to the (gSQG) model for the doubly connected case.

4) In the setting of the vortex patches the global existence with smooth boundaries is not known for $\alpha \in] 0,2[$. The simply connected V-states discussed in [8,20] offer a first class of global solutions which are periodic in time. We find here a second class of global solutions which are the doubly connected V-states.

The remainder of the chapter is organized as follows. In the next section, we shall write down the boundary equations through the conformal parametrization. In Section 3, we shall introduce and review some background material on the bifurcation theory and Gauss hypergeometric functions. In Section 4, we will study the regularity of the nonlinear functionals involved in the boundary equations. In Section 5, we conduct the spectral study and formulate the suitable assumptions to get a Fredholm operator of zero index. In Section 6 we prove Theorem 1.1. Finally, the last section will be devoted to some numerical experiments dealing with the simply and doubly connected V-states.

Notation. We need to fix some notation that will be frequently used along this chapter.

- We denote by $\mathrm{C}$ any positive constant that may change from line to line.

- For any positive real numbers $A$ and $B$, the notation $A \lesssim B$ means that there exists a positive constant $C$ independent of $A$ and $B$ such that $A \leq C B$.

- We denote by $\mathbb{D}$ the unit disc. Its boundary, the unit circle, is denoted by $\mathbb{T}$.

- Let $f: \mathbb{T} \rightarrow \mathbb{C}$ be a continuous function. We define its mean value by,

$$
f_{\mathbb{T}} f(\tau) d \tau \triangleq \frac{1}{2 i \pi} \int_{\mathbb{T}} f(\tau) d \tau
$$

where $d \tau$ stands for the complex integration.

- Let $X$ and $Y$ be two normed spaces. We denote by $\mathcal{L}(X, Y)$ the space of all continuous linear maps $T: X \rightarrow Y$ endowed with its usual strong topology.

- For a linear operator $T: X \rightarrow Y$, we denote by $N(T)$ and $R(T)$ the kernel and the range of $T$, respectively.

- If $Y$ is a vector space and $R$ is a subspace, then $Y / R$ denotes the quotient space. 


\section{Boundary EQUATIONS}

Before proceeding further with the consideration of the V-states, we shall recall Riemann mapping theorem which is one of the most important results in complex analysis. To restate this result we need to recall the definition of simply connected domains. Let $\widehat{\mathbb{C}} \triangleq \mathbb{C} \cup\{\infty\}$ denote the Riemann sphere. We say that a domain $\Omega \subset \widehat{\mathbb{C}}$ is simply connected if the set $\widehat{\mathbb{C}} \backslash \Omega$ is connected.

Riemann Mapping Theorem. Let $\mathbb{D}$ denote the unit open ball and $\Omega \subset \mathbb{C}$ be a simply connected bounded domain. Then there is a unique bi-holomorphic map called also conformal map, $\Phi: \mathbb{C} \backslash \overline{\mathbb{D}} \rightarrow \mathbb{C} \backslash \bar{\Omega}$ taking the form

$$
\Phi(z)=a z+\sum_{n \in \mathbb{N}} \frac{a_{n}}{z^{n}} \quad \text { with } \quad a>0 .
$$

In this theorem the regularity of the boundary has no effect regarding the existence of the conformal mapping but it contributes in the boundary behavior of the conformal mapping, see for instance $[38,43]$. Here, we shall recall the following result.

Kellogg-Warschawski's theorem. It can be found in [43] or in [38, Theorem 3.6]. It asserts that if the conformal map $\Phi: \mathbb{C} \backslash \overline{\mathbb{D}} \rightarrow \mathbb{C} \backslash \bar{\Omega}$ has a continuous extension to $\mathbb{C} \backslash \mathbb{D}$ which is of class $C^{n+1+\beta}$, with $n \in \mathbb{N}$ and $0<\beta<1$, then the boundary $\Phi(\mathbb{T})$ is a Jordan curve of class $C^{n+1+\beta}$.

Next, we shall write down the equation governing the boundary of the doubly connected V-states. Let $D=D_{1} \backslash D_{2}$ be a doubly connected domain, that is, $D_{1}$ and $D_{2}$ are two simply connected domains with $D_{2} \subset D_{1}$. Denote by $\Gamma_{1}$ and $\Gamma_{2}$ their boundaries, respectively. Consider the parametrization by the conformal mapping: $\phi_{j}: \mathbb{D}^{c} \rightarrow D_{j}^{c}$ satisfying

$$
\phi_{1}(z)=z+f_{1}(z)=z\left(1+\sum_{n=1}^{\infty} \frac{a_{n}}{z^{n}}\right)
$$

and

$$
\phi_{2}(z)=b z+f_{2}(z)=z\left(b+\sum_{n=1}^{\infty} \frac{b_{n}}{z^{n}}\right), \quad 0<b<1 .
$$

Now assume that $\theta_{0}=\chi_{D}$ is a rotating patch for the model (1.1) then according to [20] the boundary equations are given by

$$
\begin{aligned}
\Omega \operatorname{Re}\left\{z \overline{z^{\prime}}\right\} & =C_{\alpha} \operatorname{Im}\left\{\frac{1}{2 \pi} \int_{\partial D} \frac{d \zeta}{|z-\zeta|^{\alpha}} \overline{z^{\prime}}\right\}, \quad \forall z \in \partial D=\Gamma_{1} \cup \Gamma_{2} . \\
& =C_{\alpha} \operatorname{Im}\left\{\left(\frac{1}{2 \pi} \int_{\Gamma_{1}} \frac{d \zeta}{|z-\zeta|^{\alpha}}-\frac{1}{2 \pi} \int_{\Gamma_{2}} \frac{d \zeta}{|z-\zeta|^{\alpha}}\right) \overline{z^{\prime}}\right\},
\end{aligned}
$$

where $z^{\prime}$ denotes a tangent vector to the boundary $\partial D$ at the point $z$. We shall now rewrite the equations by using the conformal parametrizations $\phi_{1}$ and $\phi_{2}$. First remark that for $w \in \mathbb{T}$ a tangent vector on the boundary $\Gamma_{j}$ at the point $\phi_{j}(w)$ is given by

$$
\overline{z^{\prime}}=-i \bar{w} \overline{\phi_{j}^{\prime}(w)}
$$


Inserting this into the equation (2.1) and using the change of variables $\tau=\phi_{j}(w)$ give

$$
\forall w \in \mathbb{T}, \quad F_{j}\left(\Omega, \phi_{1}, \phi_{2}\right)(w)=0 ; \quad j=1,2,
$$

with

$$
\begin{aligned}
& F_{j}\left(\Omega, \phi_{1}, \phi_{2}\right)(w) \triangleq \Omega \operatorname{Im}\left\{\phi_{j}(w) \bar{w} \overline{\phi_{j}^{\prime}(w)}\right\} \\
+ & C_{\alpha} \operatorname{Im}\left\{\left(f_{\mathbb{T}} \frac{\phi_{2}^{\prime}(\tau) d \tau}{\left|\phi_{j}(w)-\phi_{2}(\tau)\right|^{\alpha}}-f_{\mathbb{T}} \frac{\phi_{1}^{\prime}(\tau) d \tau}{\left|\phi_{j}(w)-\phi_{1}(\tau)\right|^{\alpha}}\right) \bar{w} \overline{\phi_{j}^{\prime}(w)}\right\}
\end{aligned}
$$

and $\quad C_{\alpha} \triangleq \frac{\Gamma(\alpha / 2)}{2^{1-\alpha} \Gamma\left(\frac{2-\alpha}{2}\right)}$. We shall introduce the functionals

$$
G_{j}\left(\Omega, f_{1}, f_{2}\right) \triangleq F_{j}\left(\Omega, \phi_{1}, \phi_{2}\right) \quad j=1,2 .
$$

Then equations of the $\mathrm{V}$-states become,

$$
\forall w \in \mathbb{T}, \quad G_{j}\left(\Omega, f_{1}, f_{2}\right)(w)=0, \quad j=1,2 .
$$

Now it is easy to ascertain that the annulus is a rotating patch for any $\Omega \in \mathbb{R}$. Indeed, replacing $\phi_{1}$ and $\phi_{2}$ in (2.2) by Id and $b \mathrm{Id}$, respectively, we get

$$
F_{1}(\Omega, \operatorname{Id}, b \operatorname{Id})(w)=C_{\alpha} \operatorname{Im}\left\{b \bar{w} f_{\mathbb{T}} \frac{d \tau}{|w-b \tau|^{\alpha}}-\bar{w} f_{\mathbb{T}} \frac{d \tau}{|w-\tau|^{\alpha}}\right\} .
$$

Using the change of variables $\tau=w \zeta$ in the two preceding integrals we find

$$
F_{1}(\Omega, \operatorname{Id}, b \operatorname{Id})(w)=C_{\alpha} \operatorname{Im}\left\{b f_{\mathbb{T}} \frac{d \zeta}{|1-b \zeta|^{\alpha}}-f_{\mathbb{T}} \frac{d \zeta}{|1-\zeta|^{\alpha}}\right\} .
$$

Note that each integral in the right side is real since,

$$
\begin{aligned}
& \forall a \in(0,1], \quad \overline{f_{\mathbb{T}} \frac{d \zeta}{\mid 1-a \zeta^{\alpha}}}=-f_{\mathbb{T}} \frac{d \bar{\zeta}}{|1-a \bar{\zeta}|^{\alpha}} \\
& =f_{\mathbb{T}} \frac{d \xi}{|1-a \xi|^{\alpha}} \text {. }
\end{aligned}
$$

Therefore we obtain,

$$
\forall w \in \mathbb{T}, \quad F_{1}(\Omega, \operatorname{Id}, b \operatorname{Id})(w)=0 .
$$

Arguing similarly for the second component $F_{2}$ we get for any $w \in \mathbb{T}$,

$$
F_{2}(\Omega, \operatorname{Id}, b \operatorname{Id})(w)=C_{\alpha} \operatorname{Im}\left\{b^{1-\alpha} f_{\mathbb{T}} \frac{d \zeta}{|1-\zeta|^{\alpha}}-f_{\mathbb{T}} \frac{d \zeta}{|b-\zeta|^{\alpha}}\right\}=0,
$$

which is the desired result. 


\section{TOOLS}

In this section we shall recall in the first part some simple facts about Hölder spaces on the unit circle $\mathbb{T}$. In the second part we state Crandall-Rabinowitz's Theorem which is a crucial tool in the proof of Theorem 1.1. We shall also recall some important properties of the hypergeometric functions which appear in a natural way in the spectral study of the linearized operator. The last part is devoted to the computations of some integrals used later in the spectral study.

3.1. Functional spaces. Throughout this chapter it is more convenient to think of $2 \pi$ periodic function $g: \mathbb{R} \rightarrow \mathbb{C}$ as a function of the complex variable $w=e^{i \eta}$. To be more precise, let $f: \mathbb{T} \rightarrow \mathbb{R}^{2}$, be a continuous function, then it can be assimilated to a $2 \pi-$ periodic function $g: \mathbb{R} \rightarrow \mathbb{R}$ via the relation

$$
f(w)=g(\eta), \quad w=e^{i \eta} .
$$

Hence when $f$ is smooth enough we get

$$
f^{\prime}(w) \triangleq \frac{d f}{d w}=-i e^{-i \eta} g^{\prime}(\eta)
$$

Because $d / d w$ and $d / d \eta$ differ only by a smooth factor with modulus one we shall in the sequel work with $d / d w$ instead of $d / d \eta$ which appears more suitable in the computations. Moreover, if $f$ has real Fourier coefficients and is of class $C^{1}$ then we can easily check that

$$
\{\bar{f}\}^{\prime}(w)=-\frac{1}{w^{2}} \overline{f^{\prime}(w)} .
$$

Now we shall introduce Hölder spaces on the unit circle $\mathbb{T}$.

Definition 1. Let $0<\gamma<1$. We denote by $C^{\gamma}(\mathbb{T})$ the space of continuous functions $f$ such that

$$
\|f\|_{C^{\gamma}(\mathbb{T})} \triangleq\|f\|_{L^{\infty}(\mathbb{T})}+\sup _{\tau \neq w \in \mathbb{T}} \frac{|f(\tau)-f(w)|}{|\tau-w|^{\alpha}}<\infty .
$$

For any integer $n$, the space $C^{n+\gamma}(\mathbb{T})$ stands for the set of functions $f$ of class $C^{n}$ whose $n$-th order derivatives are Hölder continuous with exponent $\gamma$. It is equipped with the usual norm,

$$
\|f\|_{C^{n+\gamma}(\mathbb{T})} \triangleq\|f\|_{L^{\infty}(\mathbb{T})}+\left\|\frac{d^{n} f}{d w^{n}}\right\|_{C^{\gamma}(\mathbb{T})}
$$

Recall that the Lipschitz semi-norm is defined by,

$$
\|f\|_{\operatorname{Lip}(\mathbb{T})}=\sup _{\tau \neq w \in \mathbb{T}} \frac{|f(\tau)-f(w)|}{|\tau-w|} .
$$

Now we list some classical properties that will be used later at many places.

(1) For $n \in \mathbb{N}, \gamma \in] 0,1\left[\right.$ the space $C^{n+\gamma}(\mathbb{T})$ is an algebra.

(2) For $K \in L^{1}(\mathbb{T})$ and $f \in C^{n+\gamma}(\mathbb{T})$ we have the convolution law,

$$
\|K * f\|_{C^{n+\gamma}(\mathbb{T})} \leq\|K\|_{L^{1}(\mathbb{T})}\|f\|_{C^{n+\gamma}(\mathbb{T})} .
$$


3.2. Elements of the bifurcation theory. We intend now to recall Crandall-Rabinowitz's Theorem which is a basic tool of the bifurcation theory and will be useful in the proof of Theorem 1.1. Let $F: \mathbb{R} \times X \rightarrow Y$ be a continuous function with $X$ and $Y$ being two Banach spaces. Assume that $F(\lambda, 0)=0$ for any $\lambda$ belonging in a non empty interval $I$. Whether close to a trivial solution $\left(\lambda_{0}, 0\right)$ we can find a branch of non trivial solutions of the equation $F(\lambda, x)=0$ is the main concern of the bifurcation theory. If this happens we say that we have a bifurcation at the point $\left(\lambda_{0}, 0\right)$. We shall restrict ourselves here to the classical result of Crandall and Rabinowitz [15]. For more general results we refer the reader to the book of Kielhöfer [28].

Theorem 3.1. Let $X, Y$ be two Banach spaces, $V$ a neighborhood of 0 in $X$ and let $F$ : $\mathbb{R} \times V \rightarrow Y$ with the following properties:

(1) $F(\lambda, 0)=0$ for any $\lambda \in \mathbb{R}$.

(2) The partial derivatives $F_{\lambda}, F_{x}$ and $F_{\lambda x}$ exist and are continuous.

(3) $N\left(\mathcal{L}_{0}\right)$ and $Y / R\left(\mathcal{L}_{0}\right)$ are one-dimensional.

(4) Transversality assumption: $\partial_{\lambda} \partial_{x} F(0,0) x_{0} \notin R\left(\mathcal{L}_{0}\right)$, where

$$
N\left(\mathcal{L}_{0}\right)=\operatorname{span}\left\{x_{0}\right\}, \quad \mathcal{L}_{0} \triangleq \partial_{x} F(0,0) .
$$

If $Z$ is any complement of $N\left(\mathcal{L}_{0}\right)$ in $X$, then there is a neighborhood $U$ of $(0,0)$ in $\mathbb{R} \times X$, an interval $(-a, a)$, and continuous functions $\varphi:(-a, a) \rightarrow \mathbb{R}, \psi:(-a, a) \rightarrow Z$ such that $\varphi(0)=0, \psi(0)=0$ and

$$
F^{-1}(0) \cap U=\left\{\left(\varphi(\xi), \xi x_{0}+\xi \psi(\xi)\right) ;|\xi|<a\right\} \cup\{(\lambda, 0) ;(\lambda, 0) \in U\} .
$$

3.3. Special functions. We shall give a short introduction on the Gauss hypergeometric functions and discuss some of their basic properties. The formulae listed below will be crucial in the computations of the linearized operator associated to the $\mathrm{V}$-states equations. Recall that for any real numbers $a, b \in \mathbb{R}, c \in \mathbb{R} \backslash(-\mathbb{N})$ the hypergeometric function $z \mapsto F(a, b ; c ; z)$ is defined on the open unit disc $\mathbb{D}$ by the power series

$$
F(a, b ; c ; z)=\sum_{n=0}^{\infty} \frac{(a)_{n}(b)_{n}}{(c)_{n}} \frac{z^{n}}{n !}, \quad \forall z \in \mathbb{D} .
$$

Here, $(x)_{n}$ is the Pochhammer symbol defined by,

$$
(x)_{n}= \begin{cases}1 & n=0 \\ x(x+1) \cdots(x+n-1) & n \geq 1\end{cases}
$$

It is obvious that

$$
(x)_{n}=x(1+x)_{n-1}, \quad(x)_{n+1}=(x+n)(x)_{n} .
$$

For a future use we recall an integral representation of the hypergeometric function, for instance see [39, p. 47]. Assume that $\operatorname{Re}(c)>\operatorname{Re}(b)>0$, then

$$
F(a, b ; c ; z)=\frac{\Gamma(c)}{\Gamma(b) \Gamma(c-b)} \int_{0}^{1} x^{b-1}(1-x)^{c-b-1}(1-z x)^{-a} \mathrm{~d} x, \quad|z|<1 .
$$

The function $\Gamma: \mathbb{C} \backslash\{-\mathbb{N}\} \rightarrow \mathbb{C}$ refers to the gamma function which is the analytic continuation to the negative half plane of the usual gamma function defined on the positive 
half-plane $\{\operatorname{Re} z>0\}$ by the integral representation:

$$
\Gamma(z)=\int_{0}^{+\infty} t^{z-1} e^{-t} \mathrm{~d} t .
$$

It satisfies the relation

$$
\Gamma(z+1)=z \Gamma(z), \quad \forall z \in \mathbb{C} \backslash(-\mathbb{N}) .
$$

From this we deduce the identities

$$
(x)_{n}=\frac{\Gamma(x+n)}{\Gamma(x)}, \quad(x)_{n}=(-1)^{n} \frac{\Gamma(1-x)}{\Gamma(1-x-n)},
$$

provided all the quantities in the right terms are well-defined. Later we need the following values,

$$
\Gamma(n+1)=n !, \quad \Gamma(1 / 2)=\sqrt{\pi} .
$$

Another useful identity is the Euler's reflection formula,

$$
\Gamma(1-z) \Gamma(z)=\frac{\pi}{\sin (\pi z)}, \quad \forall z \notin \mathbb{Z}
$$

Now we shall introduce the digamma function which is nothing but the logarithmic derivative of the gamma function and often denoted by $\digamma$. It is given by

$$
\digamma(z)=\frac{\Gamma^{\prime}(z)}{\Gamma(z)}, \quad z \in \mathbb{C} \backslash(-\mathbb{N}) .
$$

The following identity is classical,

$$
\forall n \in \mathbb{N}, \quad \digamma\left(n+\frac{1}{2}\right)=-\gamma-2 \ln 2+2 \sum_{k=0}^{n-1} \frac{1}{2 k+1} .
$$

When $\operatorname{Re}(c-a-b)>0$ then it can be shown that the hypergeometric series is absolutely convergent on the closed unit disc and one has the expression,

$$
F(a, b ; c ; 1)=\frac{\Gamma(c) \Gamma(c-a-b)}{\Gamma(c-a) \Gamma(c-b)} .
$$

the proof can be found in [39, p. 49],

Now recall the Kummer's quadratic transformation

$$
F\left(a, b ; 2 b ; \frac{4 z}{(1+z)^{2}}\right)=(1+z)^{2 a} F\left(a, a+\frac{1}{2}-b ; b+\frac{1}{2} ; z^{2}\right), \quad \forall z \in[0,1[.
$$

Next we recall some contiguous function relations of the hypergeometric series, see [39].

$$
\begin{gathered}
c(c+1) F(a, b ; c ; z)-c(c+1) F(a, b ; c+1 ; z)-a b z F(a+1, b+1 ; c+2 ; z)=0, \\
c F(a, b ; c ; z)-c F(a+1, b ; c ; z)+b z F(a+1, b+1 ; c+1 ; z)=0, \\
c F(a, b ; c ; z)-c F(a, b+1 ; c ; z)+a z F(a+1, b+1 ; c+1 ; z)=0, \\
c F(a, b ; c ; z)-(c-b) F(a, b ; c+1 ; z)-b F(a, b+1 ; c+1 ; z)=0, \\
c F(a, b ; c ; z)-(c-a) F(a, b ; c+1 ; z)-a F(a+1, b ; c+1 ; z)=0, \\
12
\end{gathered}
$$




$$
\begin{gathered}
b F(a, b+1 ; c ; z)-a F(a+1, b ; c ; z)+(a-b) F(a, b ; c ; z)=0, \\
(b-a)(1-z) F(a, b ; c ; z)-(c-a) F(a-1, b ; c ; z)+(c-b) F(a, b-1 ; c ; z)=0 .
\end{gathered}
$$

Now we close this discussion with recalling Bessel function $J_{n}$ of the first kind of index $n \in \mathbb{N}$ and review some important identities. It is defined in the full space $\mathbb{C}$ by the power series

$$
J_{n}(z)=\sum_{k \geq 0} \frac{(-1)^{k}}{k !(n+k) !}\left(\frac{z}{2}\right)^{2 k+n} .
$$

The following identity called Sonine-Schafheitlin's formula will be very useful later.

$$
\begin{aligned}
\int_{0}^{+\infty} \frac{J_{\mu}(a t) J_{\nu}(b t)}{t^{\lambda}} \mathrm{d} t & =\frac{a^{\lambda-\nu-1} b^{\nu} \Gamma\left(\frac{1}{2} \mu+\frac{1}{2} \nu-\frac{1}{2} \lambda+\frac{1}{2}\right)}{2^{\lambda} \Gamma(\nu+1) \Gamma\left(\frac{1}{2} \lambda+\frac{1}{2} \mu-\frac{1}{2} \nu+\frac{1}{2}\right)} \\
& \times F\left(\frac{\mu+\nu-\lambda+1}{2}, \frac{\nu-\lambda-\mu+1}{2} ; \nu+1 ; \frac{b^{2}}{a^{2}}\right),
\end{aligned}
$$

provided that $0<b<a$ and that the integral is convergent. A detailed proof of this result can be found in [44, p. 401].

3.4. Basic integrals. The main goal of this paragraph is to compute explicitly some integrals that will appear later in the spectral study.

Lemma 3.2. Let $\alpha, b \in(0,1)$ and $n \in \mathbb{N}$. Then for any $w \in \mathbb{T}$ we have the following formulae:

$$
\begin{aligned}
f_{\mathbb{T}} \frac{\tau^{n-1}}{|w-b \tau|^{\alpha}} d \tau=w^{n} b^{n} \frac{\left(\frac{\alpha}{2}\right)_{n}}{n !} F\left(\frac{\alpha}{2}, n+\frac{\alpha}{2} ; n+1 ; b^{2}\right) . \\
f_{\mathbb{T}} \frac{(w-b \tau)\left(a w^{n}-c \tau^{n}\right)}{|w-b \tau|^{\alpha+2}} d \tau=w^{n+2} b\left[a\left(1+\frac{\alpha}{2}\right) F\left(\frac{\alpha}{2}, 2+\frac{\alpha}{2} ; 2 ; b^{2}\right)\right. \\
\left.-c b^{n} \frac{\left(1+\frac{\alpha}{2}\right)_{n+1}}{(n+1) !} F\left(\frac{\alpha}{2}, n+2+\frac{\alpha}{2} ; n+2 ; b^{2}\right)\right] .
\end{aligned}
$$

$$
\begin{aligned}
f_{\mathbb{T}} \frac{(\bar{w}-b \bar{\tau})\left(a \bar{w}^{n}-c \bar{\tau}^{n}\right)}{|w-b \tau|^{\alpha+2}} d \tau=\bar{w}^{n}[ & a b \frac{\alpha}{2} F\left(\frac{\alpha}{2}+1, \frac{\alpha}{2}+1 ; 2 ; b^{2}\right) \\
& \left.-c b^{n-1} \frac{\left(1+\frac{\alpha}{2}\right)_{n-1}}{(n-1) !} F\left(\frac{\alpha}{2}, n+\frac{\alpha}{2} ; n ; b^{2}\right)\right]
\end{aligned}
$$

$$
\begin{aligned}
f_{\mathbb{T}} \frac{(b w-\tau)\left(a w^{n}-c \tau^{n}\right)}{|b w-\tau|^{\alpha+2}} d \tau=-w^{n+2} b^{2}[ & a \frac{\alpha}{4}\left(\frac{\alpha}{2}+1\right) F\left(1+\frac{\alpha}{2}, 2+\frac{\alpha}{2} ; 3 ; b^{2}\right) \\
& \left.-c b^{n} \frac{\left(\frac{\alpha}{2}\right)_{n+2}}{(n+2) !} F\left(1+\frac{\alpha}{2}, n+2+\frac{\alpha}{2} ; n+3 ; b^{2}\right)\right] .
\end{aligned}
$$




$$
\begin{aligned}
f_{\mathbb{T}} \frac{(b \bar{w}-\bar{\tau})\left(a \bar{w}^{n}-c \bar{\tau}^{n}\right)}{|b w-\tau|^{\alpha+2}} d \tau=-\bar{w}^{n}[ & a F\left(\frac{\alpha}{2}, \frac{\alpha}{2}+1 ; 1 ; b^{2}\right) \\
& \left.-c b^{n} \frac{\left(\frac{\alpha}{2}\right)_{n}}{n !} F\left(\frac{\alpha}{2}+1, n+\frac{\alpha}{2} ; n+1 ; b^{2}\right)\right] .
\end{aligned}
$$

Proof. To prove the first identity we use successively the change of variables $\tau=w \zeta$ and $\zeta=e^{i \eta}$,

$$
\begin{aligned}
f_{\mathbb{T}} \frac{\tau^{n-1}}{|w-b \tau|^{\alpha}} d \tau & =w^{n} f_{\mathbb{T}} \frac{\zeta^{n-1}}{|1-b \zeta|^{\alpha}} d \zeta \\
& =w^{n} \frac{1}{2(1+b)^{\alpha} \pi} \int_{0}^{2 \pi} \frac{e^{i n \eta}}{\left(1-\frac{4 b}{(1+b)^{2}} \cos ^{2}(\eta / 2)\right)^{\frac{\alpha}{2}}} d \eta .
\end{aligned}
$$

Again by the change of variables $\eta / 2 \mapsto \eta$ one gets

$$
f_{\mathbb{T}} \frac{\tau^{n-1}}{|w-b \tau|^{\alpha}} d \tau=w^{n} \frac{1}{(1+b)^{\alpha} \pi} \int_{0}^{\pi} \frac{e^{i 2 n \eta}}{\left(1-\frac{4 b}{(1+b)^{2}} \cos ^{2} \eta\right)^{\frac{\alpha}{2}}} d \eta .
$$

Since $\left|\frac{4 b}{(1+b)^{2}} \cos ^{2} \eta\right|<1$ then we can use the Taylor series

$$
\left(1-\frac{4 b}{(1+b)^{2}} \cos ^{2} \eta\right)^{-\alpha / 2}=\sum_{m=0}^{\infty} \frac{(\alpha / 2)_{m}}{m !} \frac{2^{2 m} b^{m}}{(1+b)^{2 m}} \cos ^{2 m} \eta .
$$

Consequently, we get

$$
f_{\mathbb{T}} \frac{\tau^{n-1}}{|w-b \tau|^{\alpha}} d \tau=w^{n} \frac{1}{\pi(1+b)^{\alpha}} \sum_{m=0}^{\infty} \frac{(\alpha / 2)_{m}}{m !} \frac{2^{2 m} b^{m}}{(1+b)^{2 m}} \int_{0}^{\pi} \cos ^{2 m} \eta e^{i 2 n \eta} d \eta .
$$

We shall now recall the following identity, see for instance [34, p. 8] and [44, p. 449],

$$
\int_{0}^{\pi} \cos ^{x}(\eta) e^{i y \eta} d \eta=\frac{\pi \Gamma(x+1)}{2^{x} \Gamma\left(1+\frac{x+y}{2}\right) \Gamma\left(1+\frac{x-y}{2}\right)}, \quad \forall x>-1, \quad \forall y \in \mathbb{R} .
$$

As it was pointed before the gamma function has no real zeros but simple poles located at $-\mathbb{N}$ and therefore the function $\frac{1}{\Gamma}$ admits an analytic continuation on $\mathbb{C}$. Apply this formula with $x=2 m$ and $y=2 n$ yields,

Hence,

$$
\frac{1}{\pi} \int_{0}^{\pi} \cos ^{2 m}(\eta) e^{2 i n \eta} d \eta=\frac{\Gamma(2 m+1)}{2^{2 m} \Gamma(m+n+1) \Gamma(m-n+1)} .
$$

$$
\begin{aligned}
f_{\mathbb{T}} \frac{\tau^{n-1}}{|w-b \tau|^{\alpha}} d \tau & =w^{n} \frac{1}{(1+b)^{\alpha}} \sum_{m=n}^{\infty} \frac{(\alpha / 2)_{m}}{m !} \frac{\Gamma(2 m+1)}{\Gamma(m+n+1) \Gamma(m-n+1)} \frac{b^{m}}{(1+b)^{2 m}} \\
& =w^{n} \frac{1}{(1+b)^{\alpha}} \sum_{m=0}^{\infty} \frac{(\alpha / 2)_{m+n}}{(m+n) !} \frac{\Gamma(2 m+2 n+1)}{\Gamma(m+2 n+1) \Gamma(m+1)} \frac{b^{m+n}}{(1+b)^{2(m+n)}} .
\end{aligned}
$$

We shall use Legendre's duplication formula,

$$
\Gamma(z) \Gamma\left(z+\frac{1}{2}\right)=2^{1-2 z} \sqrt{\pi} \Gamma(2 z), \quad z \in \mathbb{C} \backslash\{-\mathbb{N}\},
$$


which gives

$$
\begin{aligned}
\frac{\Gamma(2 m+2 n+1)}{(m+n) !} & =\frac{\Gamma(2 m+2 n+1)}{\Gamma(m+n+1)} \\
& =\frac{2^{2 m+2 n}}{\sqrt{\pi}} \Gamma(m+n+1 / 2) .
\end{aligned}
$$

Therefore using the identity (3.5) and $\Gamma(1 / 2)=\sqrt{\pi}$ we find

$$
\frac{\Gamma(2 m+2 n+1)}{(m+n) !}=2^{2 m+2 n}(1 / 2)_{m+n} .
$$

From the elementary fact

$$
(x)_{m+n}=(x)_{n}(n+x)_{m} \quad \forall x \in \mathbb{R}
$$

one deuces

$$
f_{\mathbb{T}} \frac{\tau^{n-1}}{|w-b \tau|^{\alpha}} d \tau=w^{n} \frac{\left(\frac{\alpha}{2}\right)_{n}\left(\frac{1}{2}\right)_{n}}{(1+b)^{\alpha}(2 n) !}\left(\frac{4 b}{(1+b)^{2}}\right)^{n} \sum_{m=0}^{\infty} \frac{\left(n+\frac{\alpha}{2}\right)_{m}(n+1 / 2)_{m}}{(2 n+1)_{m} m !} \frac{2^{2 m} b^{m}}{(1+b)^{2 m}} .
$$

By definition of the hypergeometric series we conclude that

$$
f_{\mathbb{T}} \frac{\tau^{n-1}}{|w-b \tau|^{\alpha}} d \tau=w^{n} \frac{\left(\frac{\alpha}{2}\right)_{n}}{n !} \frac{2^{2 n} b^{n}}{(1+b)^{2 n+\alpha}} F\left(n+\frac{\alpha}{2}, n+\frac{1}{2} ; 2 n+1 ; \frac{4 b}{(1+b)^{2}}\right) .
$$

Using Kummer's quadratic transformation (3.10) the last identity becomes

$$
f_{\mathbb{T}} \frac{\tau^{n-1}}{|w-b \tau|^{\alpha}} d \tau=w^{n} b^{n} \frac{\left(\frac{\alpha}{2}\right)_{n}}{n !} F\left(\frac{\alpha}{2}, n+\frac{\alpha}{2} ; n+1 ; b^{2}\right) .
$$

This completes the proof of (3.19).

We intend now to compute the second integral (3.20). To this end we use the change of variables as before,

$$
\begin{aligned}
I_{n} & \triangleq f_{\mathbb{T}} \frac{(w-b \zeta)\left(a w^{n}-c \zeta^{n}\right)}{|w-b \zeta|^{\alpha+2}} d \zeta \\
& =w^{n+2} f_{\mathbb{T}} \frac{(1-b \zeta)\left(a-c \zeta^{n}\right)}{|1-b \zeta|^{\alpha+2}} d \zeta \\
& =w^{n+2}\left(a A_{0}-c A_{n}\right)
\end{aligned}
$$

where

$$
A_{n} \triangleq f_{\mathbb{T}} \frac{\zeta^{n} d \zeta}{|1-b \zeta|^{\alpha+2}}-b f_{\mathbb{T}} \frac{\zeta^{n+1} d \zeta}{|1-b \zeta|^{\alpha+2}}
$$

It follows from the identity (3.19) that

$$
\begin{aligned}
A_{n}=b^{n+1} \frac{\left(1+\frac{\alpha}{2}\right)_{n+1}}{(n+1) !}[ & F\left(\frac{\alpha}{2}+1, n+2+\frac{\alpha}{2} ; n+2 ; b^{2}\right) \\
& \left.-\frac{\left(\frac{\alpha}{2}+n+2\right)}{n+2} b^{2} F\left(\frac{\alpha}{2}+1, n+3+\frac{\alpha}{2} ; n+3 ; b^{2}\right)\right] .
\end{aligned}
$$


Then, in view of the formula (3.12) one gets

$$
A_{n}=b^{n+1} \frac{\left(1+\frac{\alpha}{2}\right)_{n+1}}{(n+1) !} F\left(\frac{\alpha}{2}, n+2+\frac{\alpha}{2} ; n+2 ; b^{2}\right) .
$$

Replacing $A_{n}$ by its expression in (3.24) we conclude that

$I_{n}=w^{n+2}\left[a b\left(1+\frac{\alpha}{2}\right) F\left(\frac{\alpha}{2}, 2+\frac{\alpha}{2} ; 2 ; b^{2}\right)-c b^{n+1} \frac{\left(1+\frac{\alpha}{2}\right)_{n+1}}{(n+1) !} F\left(\frac{\alpha}{2}, n+2+\frac{\alpha}{2} ; n+2 ; b^{2}\right)\right]$.

We shall now compute the integral (3.21). We write

$$
\begin{aligned}
K_{n} & \triangleq f_{\mathbb{T}} \frac{(\bar{w}-b \bar{\tau})\left(a \bar{w}^{n}-c \bar{\tau}^{n}\right)}{|1-b \tau|^{\alpha+2}} d \tau \\
& =\bar{w}^{n} f_{\mathbb{T}} \frac{(1-b \bar{\zeta})\left(a-c \bar{\zeta}^{n}\right)}{|1-b \zeta|^{\alpha+2}} d \zeta \\
& \triangleq \bar{w}^{n}\left(a B_{0}-c B_{n}\right) .
\end{aligned}
$$

Using the identity (3.19), $B_{0}$ can be rewritten as

$$
\begin{aligned}
B_{0} & \triangleq f_{\mathbb{T}} \frac{d \zeta}{|1-b \zeta|^{\alpha+2}}-b f_{\mathbb{T}} \frac{\bar{\zeta} d \zeta}{|1-b \zeta|^{\alpha+2}} \\
& =f_{\mathbb{T}} \frac{d \zeta}{|1-b \zeta|^{\alpha+2}}-b f_{\mathbb{T}} \frac{\zeta^{-1} d \zeta}{|1-b \zeta|^{\alpha+2}} \\
& =b\left(1+\frac{\alpha}{2}\right) F\left(\frac{\alpha}{2}+1,2+\frac{\alpha}{2} ; 2 ; b^{2}\right)-b F\left(\frac{\alpha}{2}+1, \frac{\alpha}{2}+1 ; 1 ; b^{2}\right) .
\end{aligned}
$$

Then, in view of the formula (3.14) we get

$$
B_{0}=\frac{\alpha}{2} b F\left(\frac{\alpha}{2}+1, \frac{\alpha}{2}+1 ; 2 ; b^{2}\right) .
$$

To compute $B_{n}$ we first observe that by the change of variables $\bar{\zeta} \mapsto \zeta$ we find

$$
f_{\mathbb{T}} \frac{\bar{\zeta}^{n}}{|1-b \zeta|^{\alpha+2}} d \zeta=f_{\mathbb{T}} \frac{\zeta^{n-2}}{|1-b \zeta|^{\alpha+2}} d \zeta
$$

which yields in turn

$$
\begin{aligned}
B_{n} & =A_{n-2} \\
& =b^{n-1} \frac{\left(1+\frac{\alpha}{2}\right)_{n-1}}{(n-1) !} F\left(\frac{\alpha}{2}, n+\frac{\alpha}{2} ; n ; b^{2}\right) .
\end{aligned}
$$

Consequently,

$$
K_{n}=\bar{w}^{n}\left[a b \frac{\alpha}{2} F\left(\frac{\alpha}{2}+1, \frac{\alpha}{2}+1 ; 2 ; b^{2}\right)-c b^{n-1} \frac{\left(1+\frac{\alpha}{2}\right)_{n-1}}{(n-1) !} F\left(\frac{\alpha}{2}, n+\frac{\alpha}{2} ; n ; b^{2}\right)\right] .
$$


Concerning the integral (3.22) we use a change of variable as before in order to get

$$
\begin{aligned}
P_{n} & \triangleq f_{\mathbb{T}} \frac{(b w-\zeta)\left(a w^{n}-c \zeta^{n}\right)}{|b w-\zeta|^{\alpha+2}} d \zeta \\
& =w^{n+2} f_{\mathbb{T}} \frac{(b-\zeta)\left(a-c \zeta^{n}\right)}{|b-\zeta|^{\alpha+2}} d \zeta \\
& =w^{n+2}\left(a C_{0}-c C_{n}\right)
\end{aligned}
$$

with

$$
C_{n} \triangleq b f_{\mathbb{T}} \frac{\zeta^{n} d \zeta}{|b-\zeta|^{\alpha+2}}-f_{\mathbb{T}} \frac{\zeta^{n+1} d \zeta}{|b-\zeta|^{\alpha+2}} .
$$

Observe that

$$
|b-\zeta|=|1-b \zeta| \quad \forall \zeta \in \mathbb{T} .
$$

Then, it follows from the formula (3.19) that

$$
\begin{aligned}
C_{n}=b^{n+2} \frac{\left(1+\frac{\alpha}{2}\right)_{n+1}}{(n+2) !}[ & (n+2) F\left(\frac{\alpha}{2}+1, n+2+\frac{\alpha}{2} ; n+2 ; b^{2}\right) \\
& \left.-\left(\frac{\alpha}{2}+n+2\right) F\left(\frac{\alpha}{2}+1, n+3+\frac{\alpha}{2} ; n+3 ; b^{2}\right)\right] .
\end{aligned}
$$

Using once again the identity (3.14) implies

$$
C_{n}=-b^{n+2} \frac{\left(\frac{\alpha}{2}\right)_{n+2}}{(n+2) !} F\left(1+\frac{\alpha}{2}, n+2+\frac{\alpha}{2} ; n+3 ; b^{2}\right) .
$$

Plugging the latter expression of $C_{n}$ into (3.25) yields

$$
\begin{aligned}
P_{n}=-w^{n+2}[ & a b^{2} \frac{\alpha}{4}\left(1+\frac{\alpha}{2}\right) F\left(1+\frac{\alpha}{2}, 2+\frac{\alpha}{2} ; 3 ; b^{2}\right) \\
& \left.\quad-c b^{n+2} \frac{\left(\frac{\alpha}{2}\right)_{n+2}}{(n+2) !} F\left(1+\frac{\alpha}{2}, n+2+\frac{\alpha}{2} ; n+3 ; b^{2}\right)\right] .
\end{aligned}
$$

We shall now move to the computation of the last integral (3.23),

$$
\begin{aligned}
Q_{n} & \triangleq f_{\mathbb{T}} \frac{(b \bar{w}-\bar{\tau})\left(a \bar{w}^{n}-c \bar{\tau}^{n}\right)}{|b w-\tau|^{\alpha+2}} d \tau \\
& =\bar{w}^{n} f_{\mathbb{T}} \frac{(b-\bar{\zeta})\left(a-c \bar{\zeta}^{n}\right)}{|1-b \zeta|^{\alpha+2}} d \zeta \\
& \triangleq \bar{w}^{n}\left(a D_{0}-c D_{n}\right) .
\end{aligned}
$$

From the identity (3.19) we may write

$$
\begin{aligned}
D_{0} & \triangleq b f_{\mathbb{T}} \frac{d \zeta}{|1-b \zeta|^{\alpha+2}}-f_{\mathbb{T}} \frac{\bar{\zeta} d \zeta}{|1-b \zeta|^{\alpha+2}} \\
& =b^{2}\left(1+\frac{\alpha}{2}\right) F\left(\frac{\alpha}{2}+1,2+\frac{\alpha}{2} ; 2 ; b^{2}\right)-F\left(\frac{\alpha}{2}+1, \frac{\alpha}{2}+1 ; 1 ; b^{2}\right) .
\end{aligned}
$$


Therefore by the formula (3.12) we obtain

$$
D_{0}=-F\left(\frac{\alpha}{2}, \frac{\alpha}{2}+1 ; 1 ; b^{2}\right) .
$$

To compute $D_{n}$ we write through a change of variables,

$$
\begin{aligned}
D_{n} & \triangleq b f_{\mathbb{T}} \frac{\bar{\zeta}^{n} d \zeta}{|1-b \zeta|^{\alpha+2}}-f_{\mathbb{T}} \frac{\bar{\zeta}^{n+1} d \zeta}{|1-b \zeta|^{\alpha+2}} \\
& =b f_{\mathbb{T}} \frac{\zeta^{n-2} d \zeta}{|1-b \zeta|^{\alpha+2}}-f_{\mathbb{T}} \frac{\zeta^{n-1} d \zeta}{|1-b \zeta|^{\alpha+2}} \\
& =C_{n-2},
\end{aligned}
$$

which implies in view of (3.26)

$$
D_{n}=-b^{n} \frac{\left(\frac{\alpha}{2}\right)_{n}}{n !} F\left(1+\frac{\alpha}{2}, n+\frac{\alpha}{2} ; n+1 ; b^{2}\right) .
$$

Hence we find

$$
Q_{n}=\bar{w}^{n}\left[-a F\left(\frac{\alpha}{2}, \frac{\alpha}{2}+1 ; 1 ; b^{2}\right)+c b^{n} \frac{\left(\frac{\alpha}{2}\right)_{n}}{n !} F\left(\frac{\alpha}{2}+1, n+\frac{\alpha}{2} ; n+1 ; b^{2}\right)\right]
$$

and therefore the proof of the lemma is now complete.

\section{REGUlARITY OF THE NONLINEAR FUNCTIONAL}

This section is devoted to the regularity study of the nonlinear functional $G$ introduced in (2.3) and which defines the V-states equations. We shall check the regularity assumptions required by Crandall-Rabinowitz's Theorem. The computations are vey heavy and can be done in a straightforward way without new difficulties compared to the simply connected case treated in the paper [20]. Many of the details may be found in that work and will not be reiterated here. Therefore for the sake of concise presentation we shall study the new terms involving the interaction between the boundaries. However, regarding the self-induced terms we only recall the results from the paper [20]. To begin with, we introduce the function spaces that we shall use. We set,

$$
X=C_{a r}^{2-\alpha}(\mathbb{T}) \times C_{a r}^{2-\alpha}(\mathbb{T}), \quad Y=H \times H,
$$

with

$$
C_{a r}^{2-\alpha}(\mathbb{T})=\left\{f \in C^{2-\alpha}(\mathbb{T}) ; f(w)=\sum_{n \geq 1} a_{n} \bar{w}^{n}, w \in \mathbb{T}, a_{n} \in \mathbb{R}, n \in \mathbb{N}^{\star}\right\}
$$

and

$$
H=\left\{g \in C^{1-\alpha}(\mathbb{T}) ; g(w)=\frac{i}{2} \sum_{n \geq 1} a_{n}\left(w^{n}-\bar{w}^{n}\right), w \in \mathbb{T}, a_{n} \in \mathbb{R}, n \in \mathbb{N}\right\} .
$$

For $b \in(0,1)$, let $V$ denote the product $B_{r} \times B_{r}$, where $B_{r}$ is the open ball of $X$ with center 0 and radius $r=(1 / 4) \min \{b, 1-b\}$. We note that this choice is done in order to guarantee that $\phi_{1}=\mathrm{Id}+f_{1}$ and $\phi_{2}=b \mathrm{Id}+f_{2}$ are conformal for $f_{1}, f_{2} \in B_{r}$ and to prevent the intersection between the curves $\phi_{1}(\mathbb{T})$ and $\phi_{2}(\mathbb{T})$ which represent the boundaries of the 
V-states.

Now recall from (2.3) the form of the functional $G=\left(G_{1}, G_{2}\right)$,

$$
G_{j}\left(\Omega, f_{1}, f_{2}\right) \triangleq \operatorname{Im}\left\{\left(\Omega \phi_{j}(w)+S\left(\phi_{2}, \phi_{j}\right)(w)-S\left(\phi_{1}, \phi_{j}\right)(w)\right) \bar{w} \overline{\phi_{j}^{\prime}(w)}\right\}, \quad w \in \mathbb{T}, j=1,2,
$$

where $S$ is defined by

$$
S\left(\phi_{i}, \phi_{j}\right)(w)=C_{\alpha} f_{\mathbb{T}} \frac{\phi_{i}^{\prime}(\tau) d \tau}{\left|\phi_{j}(w)-\phi_{i}(\tau)\right|^{\alpha}}, \quad i, j=1,2 .
$$

We shall rewrite $G_{j}$ as follows,

$$
G_{j}\left(\Omega, f_{1}, f_{2}\right)=L_{j}\left(\Omega, f_{j}\right)+N_{j}\left(f_{1}, f_{2}\right), \quad j=1,2,
$$

with

and

$$
L_{j}\left(\Omega, f_{j}\right) \triangleq \operatorname{Im}\left\{\left(\Omega \phi_{j}(w)+(-1)^{j} S\left(\phi_{j}, \phi_{j}\right)(w)\right) \bar{w} \overline{\phi_{j}^{\prime}(w)}\right\}
$$

$$
N_{j}\left(f_{1}, f_{2}\right) \triangleq(-1)^{j-1} \operatorname{Im}\left\{S\left(\phi_{i}, \phi_{j}\right)(w) \bar{w} \overline{\phi_{j}^{\prime}(w)}\right\}, \quad i \neq j,
$$

usually with the notation $\phi_{1}=\operatorname{Id}+f_{1}, \phi_{2}=b \operatorname{Id}+f_{2}$.

We propose to prove the following result concerning the regularity of $G$.

Proposition 4.1. The following holds true.

(1) $G: \mathbb{R} \times V \rightarrow Y$ is well-defined.

(2) $G: \mathbb{R} \times V \rightarrow Y$ is of class $C^{1}$.

(3) The partial derivative $\partial_{\Omega} D G: \mathbb{R} \times V \rightarrow \mathcal{L}(X, Y)$ exists and is continuous.

Proof. Notice that the terms $L_{j}, j=1,2$ appears modulo the sign of $(-1)^{j}$ in the simply connected case discussed in the paper [20] and all the computations were done there. Therefore we shall restrict ourselves to recalling just the results of those computations:

(1) $L_{j}: \mathbb{R} \times B_{r} \rightarrow H$ is well-defined.

(2) $L_{j}: \mathbb{R} \times B_{r} \rightarrow H$ is of class $C^{1}$.

Moreover the differential $D L_{j}$ is given for $f_{j} \in B_{r}, h_{j} \in C_{a r}^{2-\alpha}(\mathbb{T})$ by

$$
\begin{aligned}
D L_{j}\left(\Omega, f_{j}\right) h_{j}(w)= & \operatorname{Im}\left\{\Omega\left(\bar{w} \overline{h_{j}^{\prime}(w)} \phi_{j}(w)+\bar{w} \overline{\phi_{j}^{\prime}(w)} h_{j}(w)\right)\right. \\
& +(-1)^{j} \bar{w} \overline{h_{j}^{\prime}(w)} S\left(\phi_{j}, \phi_{j}\right)(w)+(-1)^{j} C_{\alpha} \bar{w} \overline{\phi_{j}^{\prime}(w)} f \frac{h_{j}^{\prime}(\tau)}{\left|\phi_{j}(w)-\phi_{j}(\tau)\right|^{\alpha}} d \tau \\
& \left.+(-1)^{j+1} \alpha C_{\alpha} \bar{w} \overline{\phi_{j}^{\prime}(w)} f_{\mathbb{T}} \frac{\operatorname{Re}\left[\left(\phi_{j}(w)-\phi_{j}(\tau)\right)\left(\overline{h_{j}(w)}-\overline{h_{j}(\tau)}\right)\right] \phi_{j}^{\prime}(\tau)}{\left|\phi_{j}(w)-\phi_{j}(\tau)\right|^{\alpha+2}} d \tau\right\} .
\end{aligned}
$$

In addition, the partial derivative $\partial_{\Omega} D L_{j}: \mathbb{R} \times B_{r} \rightarrow \mathcal{L}\left(C_{a r}^{2-\alpha}(\mathbb{T}), H\right)$ exists and is continuous. It is given by the formula,

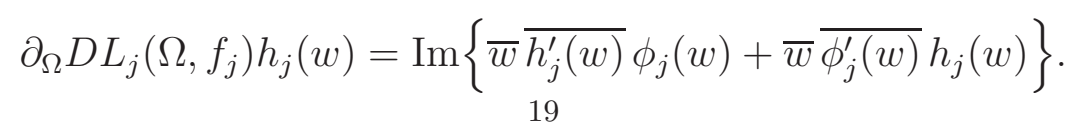


If we prove the regularity properties for the second part $N_{j}$ then we can easily deduce that

$$
\begin{aligned}
\partial_{\Omega} D G_{j}\left(\Omega, f_{1}, f_{2}\right)\left(h_{1}, h_{2}\right)(w) & =\partial_{\Omega} D L_{j}\left(\Omega, f_{j}\right) h_{j}(w) \\
& =\operatorname{Im}\left\{\bar{w} \overline{h_{j}^{\prime}(w)} \phi_{j}(w)+\bar{w} \overline{\phi_{j}^{\prime}(w)} h_{j}(w)\right\} .
\end{aligned}
$$

Therefore all the regularity assumptions are satisfied for the terms $L_{j}$ and to complete the proof of the proposition we should check these assumptions for $N_{j}$. More precisely, we shall prove that $N_{j}: V \rightarrow H$ is well-defined and is of class $C^{1}$.

(1) First, we shall prove that for $\left(f_{1}, f_{2}\right) \in V$ we have $N_{j}\left(f_{1}, f_{2}\right) \in C^{1-\alpha}(\mathbb{T})$. Because the space $C^{1-\alpha}(\mathbb{T})$ is an algebra the problem reduces to show that for $i \neq j$, the function $S\left(\phi_{i}, \phi_{j}\right)$ belongs to $C^{1-\alpha}(\mathbb{T})$. This can be deduced easily from the next general result. Let $\left(f_{1}, f_{2}\right) \in V$ and $\phi_{1}=\mathrm{Id}+f_{1}, \phi_{2}=b \mathrm{Id}+f_{2}$ and define the operator

$$
\mathcal{T} \chi(w) \triangleq f_{\mathbb{T}} \frac{\chi(\tau)}{\left|\phi_{j}(w)-\phi_{i}(\tau)\right|^{\alpha}} d \tau, \quad w \in \mathbb{T} .
$$

Then

$$
\|\mathcal{T} \chi\|_{C^{1-\alpha}(\mathbb{T})} \leq C\left\|\phi_{j}\right\|_{C^{1-\alpha}(\mathbb{T})}\|\chi\|_{L^{\infty}(\mathbb{T})}
$$

The proof of this inequality will be done in a straightforward way since as we shall see the kernel is not singular. This is due to the fact that the inner and the outer boundaries do not intersect. Indeed, for all $w, \tau \in \mathbb{T}$ we can write

$$
\begin{aligned}
\left|\phi_{1}(w)-\phi_{2}(\tau)\right| & \geq|w-b \tau|-\left|f_{1}(w)\right|-\left|f_{2}(\tau)\right| \\
& \geq(1-b)-\left\|f_{1}\right\|_{L^{\infty}}-\left\|f_{2}\right\|_{L^{\infty}}>\frac{1-b}{2} .
\end{aligned}
$$

The same result remains true if we change $\tau$ by $w$ and therefore we get for $i \neq j$

$$
\left|\phi_{i}(w)-\phi_{j}(\tau)\right| \geq \frac{1-b}{2} .
$$

It follows that

$$
|\mathcal{T} \chi(w)| \lesssim \int_{\mathbb{T}} \frac{|\chi(\tau)|}{\left|\phi_{j}(w)-\phi_{i}(\tau)\right|^{\alpha}}|d \tau| \lesssim\|\chi\|_{L^{\infty}(\mathbb{T})}
$$

which implies that

$$
\|T \chi\|_{L^{\infty}(\mathbb{T})} \leq C\|\chi\|_{L^{\infty}(\mathbb{T})} .
$$

Next take $w_{1} \neq w_{2} \in \mathbb{T}$. Using the inequality (4.5) gives

$$
\begin{aligned}
&\left|\mathcal{T} \chi\left(w_{1}\right)-\mathcal{T} \chi\left(w_{2}\right)\right| \lesssim \int_{\mathbb{T}}\left|\frac{1}{\left|\phi_{j}\left(w_{1}\right)-\phi_{i}(\tau)\right|^{\alpha}}-\frac{1}{\left|\phi_{j}\left(w_{2}\right)-\phi_{i}(\tau)\right|^{\alpha}}\right||\chi(\tau)||d \tau| \\
& \lesssim\|\chi\|_{L^{\infty}(\mathbb{T})} \int_{\mathbb{T}}|| \phi_{j}\left(w_{1}\right)-\left.\phi_{i}(\tau)\right|^{\alpha}-\left|\phi_{j}\left(w_{2}\right)-\phi_{i}(\tau)\right|^{\alpha}|| d \tau \mid \\
& \lesssim\|\chi\|_{L^{\infty}(\mathbb{T})}\left|\phi_{j}\left(w_{1}\right)-\phi_{j}\left(w_{2}\right)\right|, \\
& 20
\end{aligned}
$$


where we have used in the last estimate the following inequality: for $\alpha \in(0,1)$, there exists a constant $C>0$, such that

$$
\forall a, b \in \mathbb{R}_{+}^{*}, \quad\left|a^{\alpha}-b^{\alpha}\right| \leq C \frac{|a-b|}{a^{1-\alpha}+b^{1-\alpha}}
$$

Finally, using the fact that $\phi_{i} \in C^{2-\alpha}(\mathbb{T}) \hookrightarrow C^{1-\alpha}(\mathbb{T})$ one can conclude that

$$
\left|\mathcal{T} \chi\left(w_{1}\right)-\mathcal{T} \chi\left(w_{2}\right)\right| \lesssim\|\chi\|_{L^{\infty}(\mathbb{T})}|| \phi_{j} \|_{C^{1-\alpha}(\mathbb{T})}\left|w_{1}-w_{2}\right|^{1-\alpha}
$$

which is the desired result. Now applying (4.4) to the operator $S$ we get

$$
\begin{aligned}
\left\|S\left(\phi_{i}, \phi_{j}\right)\right\|_{C^{1-\alpha}(\mathbb{T})} & \leq C\left\|\phi_{j}\right\|_{C^{1-\alpha}(\mathbb{T})}\left\|\phi_{i}^{\prime}\right\|_{L^{\infty}(\mathbb{T})} \\
& \leq C\left\|\phi_{j}\right\|_{C^{2-\alpha}(\mathbb{T})}\left\|\phi_{i}\right\|_{C^{2-\alpha}(\mathbb{T})}
\end{aligned}
$$

To complete the proof of the first point we shall verify that the Fourier coefficients of $N_{j}\left(f_{1}, f_{2}\right)$ belong to $i \mathbb{R}$. From the definition of the space $X$ in (4.1) the mapping $\phi_{j}$ has real Fourier coefficients and thus the Fourier coefficients of $\phi_{j}^{\prime}$ are real too. Since this property is stable under the conjugation and the multiplication the problem reduces to prove that the Fourier coefficients of $S\left(\phi_{i}, \phi_{j}\right)$ are real. For this last purpose, we take the conjugate and make a change of variables,

$$
\begin{aligned}
\overline{S\left(\phi_{i}, \phi_{j}\right)(w)} & =-C_{\alpha} f_{\mathbb{T}} \frac{\phi_{i}^{\prime}(\bar{\tau}) d \bar{\tau}}{\left|\phi_{j}(\bar{w})-\phi_{i}(\bar{\tau})\right|^{\alpha}} \\
& =C_{\alpha} f_{\mathbb{T}} \frac{\phi_{i}^{\prime}(\zeta) d \zeta}{\left|\phi_{j}(\bar{w})-\phi_{i}(\zeta)\right|^{\alpha}} \\
& =S\left(\phi_{i}, \phi_{j}\right)(\bar{w}) .
\end{aligned}
$$

This proves that the Fourier coefficients of the functions $S\left(\phi_{i}, \phi_{j}\right)$ are real and the proof of the first part (1) is now achieved.

(2) The strategy to get that $N_{j}$ is of class $C^{1}$ on $V$ consists first in checking the existence of its Gâteaux derivative. Second we show that the Gâteaux derivative is strongly continuous. This will ensure in the same time the existence of Fréchet derivative and its continuity.

The Gâteaux derivative of the function $N_{j}$ at $\left(f_{1}, f_{2}\right)$ in the direction $h=\left(h_{1}, h_{2}\right) \in X$ is given by the formula

$$
\begin{aligned}
D N_{j}\left(f_{1}, f_{2}\right) h & =D_{f_{1}} N_{j}\left(f_{1}, f_{2}\right) h_{1}+D_{f_{2}} N_{j}\left(f_{1}, f_{2}\right) h_{2} \\
& \triangleq \lim _{t \rightarrow 0} \frac{1}{t}\left[N_{j}\left(f_{1}+t h_{1}, f_{2}\right)-N_{j}\left(f_{1}, f_{2}\right)\right]+\lim _{t \rightarrow 0} \frac{1}{t}\left[N_{j}\left(f_{1}, f_{2}+t h_{2}\right)-N_{j}\left(f_{1}, f_{2}\right)\right],
\end{aligned}
$$

where the limits are taken in the strong topology of $Y$. Thus we shall first prove the existence of these limit in the pointwise sense, that is for every point $w \in \mathbb{T}$, and after check that these limits exist in the strong topology of $C^{1-\alpha}(\mathbb{T})$.

Let us first check for each point $\left(f_{1}, f_{2}\right) \in V$ the existence of $D_{f_{j}} N_{j}\left(f_{1}, f_{2}\right)$ as a linear and bounded operator, that is, $D_{f_{j}} N_{j}\left(f_{1}, f_{2}\right) \in \mathcal{L}\left(C_{a r}^{2-\alpha}(\mathbb{T}), H\right)$. With the notation $\phi_{1}=\operatorname{Id}+f_{1}$ 
and $\phi_{2}=b \mathrm{Id}+f_{2}$, one has

$$
\begin{aligned}
D_{f_{j}} N_{j}\left(f_{1}, f_{2}\right) h_{j}(w)=(-1)^{j-1} \operatorname{Im}\left\{\bar{w} \overline{h_{j}^{\prime}(w)} S\left(\phi_{i}, \phi_{j}\right)(w)\right. \\
\left.+\left.\bar{w} \overline{\phi_{j}^{\prime}(w)} \frac{d}{d t}\right|_{t=0} S\left(\phi_{i}(w), \phi_{j}(w)+t h_{j}(w)\right)\right\} .
\end{aligned}
$$

We shall make use of the following identity: let $A \in \mathbb{C}^{\star}, B \in \mathbb{C}, \alpha \in \mathbb{R}$ and define the function $K: t \mapsto|A+B t|^{\alpha}$ which is smooth close to zero, then we have

$$
K^{\prime}(0)=\alpha|A|^{\alpha-2} \operatorname{Re}(\bar{A} B) .
$$

Combining this formula with few easy computations yield

$$
\begin{aligned}
\left.\frac{d}{d t}\right|_{t=0} S\left(\phi_{i}, \phi_{j}+t h_{j}\right)(w)=- & \frac{\alpha}{2} C_{\alpha}\left[\overline{h_{j}(w)} f_{\mathbb{T}} \frac{\left(\phi_{j}(w)-\phi_{i}(\tau)\right) \phi_{i}^{\prime}(\tau)}{\left|\phi_{j}(w)-\phi_{i}(\tau)\right|^{\alpha+2}} d \tau\right. \\
& \left.\quad+h_{j}(w) f_{\mathbb{T}} \frac{\left(\overline{\phi_{j}(w)}-\overline{\phi_{i}(\tau)}\right) \phi_{i}^{\prime}(\tau)}{\left|\phi_{j}(w)-\phi_{i}(\tau)\right|^{\alpha+2}} d \tau\right] \\
\triangleq & -\frac{\alpha}{2} C_{\alpha}\left[\overline{h_{j}(w)} A_{i}\left(\phi_{j}\right)(w)+h_{j}(w) B_{i}\left(\phi_{j}\right)(w)\right] .
\end{aligned}
$$

Therefore,

$$
\begin{aligned}
D_{f_{j}} N_{j}\left(\Omega, f_{1}, f_{2}\right) h_{j}(w)=(-1)^{j-1} \operatorname{Im}\{\bar{w} & \overline{h_{j}^{\prime}(w)} S\left(\phi_{i}, \phi_{j}\right)(w) \\
& \left.-\frac{\alpha}{2} C_{\alpha} \bar{w} \overline{\phi_{j}^{\prime}(w)}\left[\overline{h_{j}(w)} A_{i}\left(\phi_{j}\right)(w)+h_{j}(w) B_{i}\left(\phi_{j}\right)(w)\right]\right\} .
\end{aligned}
$$

Using the algebra structure of $C^{1-\alpha}(\mathbb{T})$ combined with the estimate (4.7), we get

$$
\left\|D_{f_{j}} N_{j}\left(f_{1}, f_{2}\right) h_{j}\right\|_{C^{1-\alpha}(\mathbb{T})} \lesssim\left(1+\left\|A_{i}\left(\phi_{j}\right)\right\|_{C^{1-\alpha}(\mathbb{T})}+\left\|B_{i}\left(\phi_{j}\right)\right\|_{C^{1-\alpha}}\right)\left\|h_{j}\right\|_{C^{2-\alpha}(\mathbb{T})} .
$$

It remains to estimate the terms $A_{i}\left(\phi_{j}\right)$ and $B_{i}\left(\phi_{j}\right)$. For the first one, we get by virtue of $(4.5)$,

$$
\begin{aligned}
\left|A_{i}\left(\phi_{j}\right)(w)\right| & \lesssim \int_{\mathbb{T}} \frac{\left|\phi_{i}^{\prime}(\tau)\right|}{\left|\phi_{j}(w)-\phi_{i}(\tau)\right|^{\alpha+1}}|d \tau| \\
& \lesssim\left\|\phi_{i}^{\prime}\right\|_{L^{\infty}(\mathbb{T})} .
\end{aligned}
$$

Now let $w_{1} \neq w_{2} \in \mathbb{T}$, then

$$
\begin{aligned}
\left|A_{i}\left(\phi_{j}\right)\left(w_{1}\right)-A_{i}\left(\phi_{j}\right)\left(w_{2}\right)\right| & \leq \int_{\mathbb{T}}\left|\frac{\overline{\phi_{j}\left(w_{1}\right)}-\overline{\phi_{i}(\tau)}}{\left|\phi_{j}\left(w_{1}\right)-\phi_{i}(\tau)\right|^{\alpha+2}}-\frac{\overline{\phi_{j}\left(w_{2}\right)}-\overline{\phi_{i}(\tau)}}{\left|\phi_{j}\left(w_{2}\right)-\phi_{i}(\tau)\right|^{2+\alpha}}\right|\left|\phi_{i}^{\prime}(\tau)\right||d \tau| \\
& \triangleq \int_{\mathbb{T}}\left|K\left(w_{1}, \tau\right)-K\left(w_{2}, \tau\right)\right|\left|\phi_{i}^{\prime}(\tau)\right||d \tau| .
\end{aligned}
$$


Few easy computations show that

$$
\begin{aligned}
\left|K\left(w_{1}, \tau\right)-K\left(w_{2}, \tau\right)\right| & \lesssim \frac{\left|\phi_{j}\left(w_{2}\right)-\phi_{j}\left(w_{1}\right)\right|}{\left|\phi_{j}\left(w_{1}\right)-\phi_{i}(\tau)\right|^{\alpha+2}} \\
& +\frac{|| \phi_{j}\left(w_{2}\right)-\left.\phi_{i}(\tau)\right|^{\alpha+2}-\left|\phi_{j}\left(w_{1}\right)-\phi_{i}(\tau)\right|^{\alpha+2} \mid}{\left|\phi_{j}\left(w_{1}\right)-\phi_{i}(\tau)\right|^{\alpha+2}\left|\phi_{j}\left(w_{2}\right)-\phi_{i}(\tau)\right|^{\alpha+1}} .
\end{aligned}
$$

Concerning the last term we shall use the following inequality whose proof is classical.

$$
\left|a^{k+1+\alpha}-b^{k+1+\alpha}\right| \leq C(k, \alpha)|a-b|\left(a^{k+\alpha}+b^{k+\alpha}\right), \quad a, b \in \mathbb{R}_{+}, k \in \mathbb{N}^{*}, 0<\alpha .
$$

Hence, we get

$$
\begin{aligned}
\frac{\left|\phi_{j}\left(w_{2}\right)-\phi_{i}(\tau)\right|^{\alpha+2}-\left|\phi_{j}\left(w_{1}\right)-\phi_{i}(\tau)\right|^{\alpha+2} \mid}{\left|\phi_{j}\left(w_{1}\right)-\phi_{i}(\tau)\right|^{\alpha+2}\left|\phi_{j}\left(w_{2}\right)-\phi_{i}(\tau)\right|^{\alpha+1}} & \lesssim \frac{\left|\phi_{j}\left(w_{2}\right)-\phi_{j}\left(w_{1}\right)\right|}{\left|\phi_{j}\left(w_{1}\right)-\phi_{i}(\tau)\right|^{\alpha+2}} \\
& +\frac{\left|\phi_{j}\left(w_{2}\right)-\phi_{j}\left(w_{1}\right)\right|}{\left|\phi_{j}\left(w_{1}\right)-\phi_{i}(\tau)\right|\left|\phi_{j}\left(w_{2}\right)-\phi_{i}(\tau)\right|^{\alpha+1}} .
\end{aligned}
$$

Inserting this in the estimate (4.14) and using the inequality (4.5) we find

$$
\begin{aligned}
\left|K\left(w_{1}, \tau\right)-K\left(w_{2}, \tau\right)\right| & \lesssim\left|\phi_{j}\left(w_{2}\right)-\phi_{j}\left(w_{1}\right)\right| \\
& \lesssim\left\|\phi_{j}\right\|_{C^{1-\alpha}}\left|w_{2}-w_{1}\right|^{1-\alpha} .
\end{aligned}
$$

Now by plugging the latter estimate into (4.13) one gets

$$
\left|A_{i}\left(\phi_{j}\right)\left(w_{1}\right)-A_{i}\left(\phi_{j}\right)\left(w_{2}\right)\right| \leq C\left|w_{2}-w_{1}\right|^{1-\alpha}
$$

which is the desired result. The estimate of the term $B_{i}\left(\phi_{j}\right)$ can be done in a similar way by observing that

$$
B_{i}\left(\phi_{j}\right)(w)=f_{\mathbb{T}} \overline{K(w, \tau)} \phi_{i}^{\prime}(\tau) d \tau
$$

Consequently, from (4.12) we deduce that

$$
\left\|D_{f_{j}} N_{j}\left(f_{1}, f_{2}\right) h_{j}\right\|_{C^{1-\alpha}(\mathbb{T})} \leq C\left\|h_{1}\right\|_{C^{2-\alpha}(\mathbb{T})} .
$$

This means that $D_{f_{j}} N_{j}\left(f_{1}, f_{2}\right) \in \mathcal{L}\left(C_{a r}^{1-\alpha}(\mathbb{T}), H\right)$.

Let us now move to the computation of $D_{f_{i}} N_{j}\left(f_{1}, f_{2}\right) h_{i}$, for $i \neq j$ when $\left(f_{1}, f_{2}\right) \in V$ and $h_{i} \in C_{a r}^{2-\alpha}(\mathbb{T})$. From the definition, we obtain the formula

$$
D_{f_{i}} N_{j}\left(f_{1}, f_{2}\right) h_{i}(w)=(-1)^{j-1} \operatorname{Im}\left\{\left.\bar{w} \overline{\phi_{j}^{\prime}(w)} \frac{d}{d t}\right|_{t=0} S\left(\phi_{i}(w)+t h_{i}(w), \phi_{j}(w)\right)\right\} .
$$


Some easy computations combined with the relation (4.10) allow to get

$$
\begin{aligned}
\left.\frac{d}{d t}\right|_{t=0} S\left(\phi_{i}(w)+t h_{i}(w), \phi_{j}(w)\right)= & C_{\alpha} f_{\mathbb{T}} \frac{h_{i}^{\prime}(\tau)}{\left|\phi_{j}(w)-\phi_{i}(\tau)\right|^{\alpha}} d \tau \\
& +\frac{\alpha}{2} C_{\alpha} f_{\mathbb{T}} \frac{\left(\overline{\phi_{j}(w)}-\overline{\phi_{i}(\tau)}\right) h_{i}(\tau) \phi_{i}^{\prime}(\tau)}{\left|\phi_{j}(w)-\phi_{i}(\tau)\right|^{\alpha+2}} d \tau \\
& +\frac{\alpha}{2} C_{\alpha} f_{\mathbb{T}} \frac{\left(\phi_{j}(w)-\phi_{i}(\tau)\right) \overline{h_{i}(\tau)} \phi_{i}^{\prime}(\tau)}{\left|\phi_{j}(w)-\phi_{i}(\tau)\right|^{\alpha+2}} d \tau \\
\triangleq & C_{i}\left(\phi_{j}, h_{i}\right)(w)+D_{i}\left(\phi_{j}, h_{i}\right)(w)+E_{i}\left(\phi_{j}, h_{i}\right)(w) .
\end{aligned}
$$

It follows that,

$$
D_{f_{i}} N_{j}\left(f_{1}, f_{2}\right) h_{i}(w)=\operatorname{Im}\left\{\bar{w} \overline{\phi_{j}^{\prime}(w)}\left[C_{i}\left(\phi_{j}, h_{i}\right)(w)+D_{i}\left(\phi_{j}, h_{i}\right)(w)+E_{i}\left(\phi_{j}, h_{i}\right)(w)\right]\right\} .
$$

Since $C^{1-\alpha}(\mathbb{T})$ is an algebra one finds that

$$
\left\|D_{f_{j}} N_{j}\left(\Omega, f_{1}, f_{2}\right) h_{j}\right\|_{C^{1-\alpha}(\mathbb{T})} \lesssim\left\|C_{i}\left(\phi_{j}, h_{i}\right)\right\|_{C^{1-\alpha}}+\left\|D_{i}\left(\phi_{j}, h_{i}\right)\right\|_{C^{1-\alpha}(\mathbb{T})}+\left\|E_{i}\left(\phi_{j}, h_{i}\right)\right\|_{C^{1-\alpha}(\mathbb{T})} .
$$

The estimate of the term $C_{i}\left(\phi_{j}, h_{i}\right)$ follows immediately from (4.4) and we get,

$$
\left\|C_{i}\left(\phi_{j}, h_{i}\right)\right\|_{C^{1-\alpha}(\mathbb{T})} \lesssim\left\|\phi_{j}\right\|_{C^{1-\alpha}(\mathbb{T})}\left\|h_{i}^{\prime}\right\|_{L^{\infty}(\mathbb{T})} .
$$

For the terms $D_{i}\left(\phi_{j}, h_{i}\right)$ and $E_{i}\left(\phi_{j}, h_{i}\right)$ we can proceed similarly as for $A_{i}\left(\phi_{j}, h_{j}\right)$ and we find

$$
\left\|D_{i}\left(\phi_{j}, h_{i}\right)\right\|_{C^{1-\alpha}(\mathbb{T})}+\left\|E_{i}\left(\phi_{j}, h_{i}\right)\right\|_{C^{1-\alpha}(\mathbb{T})} \lesssim\left\|h_{i}\right\|_{L^{\infty}(\mathbb{T})} .
$$

Putting together the preceding estimates yields,

$$
\left\|D_{f_{i}} N_{j}\left(f_{1}, f_{2}\right) h_{i}\right\|_{C^{1-\alpha}(\mathbb{T})} \leq C\left\|h_{i}\right\|_{C^{2-\alpha}(\mathbb{T})} .
$$

This shows that $D_{f_{i}} N_{j}\left(f_{1}, f_{2}\right) \in \mathcal{L}\left(C_{a r}^{1-\alpha}(\mathbb{T}), H\right)$.

To achieve the existence proof of the Gâteaux derivatives it remains to check that the convergence in (4.8) occurs in the strong topology of $C^{1-\alpha}(\mathbb{T})$. There are many terms to analyze and they can be treated in a similar way. The computations are straightforward but slightly long and we prefer just to treat a significant term and the remaining ones are quite similar. For example in the first term of the right-hand side of (4.9) we need to check

$$
\lim _{t \rightarrow 0} S\left(\phi_{i}, \phi_{j}+t h_{j}\right)-S\left(\phi_{i}, \phi_{j}\right)=0 \quad \text { in } \quad C^{1-\alpha}(\mathbb{T})
$$

To simplify the notation we set

$$
T_{i j}(t, w)=S\left(\phi_{i}, \phi_{j}+t h_{j}\right)(w)-S\left(\phi_{i}, \phi_{j}\right)(w) .
$$

Let $t>0$ such that $t\left\|h_{j}\right\|_{L^{\infty}}<(1-b) / 2$. Then by (4.2) we have

$$
\begin{aligned}
T_{i j}(t, w) & =f_{\mathbb{T}}\left(\frac{\phi_{i}^{\prime}(\tau)}{\left|\phi_{j}(w)-\phi_{i}(\tau)+t h_{j}(w)\right|^{\alpha}}-\frac{\phi_{i}^{\prime}(\tau)}{\left|\phi_{j}(w)-\phi_{i}(\tau)\right|^{\alpha}}\right) d \tau \\
& \triangleq f_{\mathbb{T}} K(t, w, \tau) \phi_{i}^{\prime}(\tau) d \tau .
\end{aligned}
$$

It follows from the inequalities (4.5) and (4.6) that

$$
|K(t, w, \tau)| \underset{24}{\lesssim} t\left\|h_{j}\right\|_{L^{\infty}(\mathbb{T})}
$$


which implies in turn,

$$
\left|T_{i j}(t, w)\right| \lesssim t\left\|h_{j}\right\|_{L^{\infty}(\mathbb{T})}
$$

Therefore we get

$$
\lim _{t \rightarrow 0}\left\|T_{i j}(t, \cdot)\right\|_{L^{\infty}(\mathbb{T})}=0
$$

Now for $w_{1} \neq w_{2} \in \mathbb{T}$, we write by the Mean Value Theorem

$$
\begin{aligned}
\left|T_{i j}\left(t, w_{1}\right)-T_{i j}\left(t, w_{2}\right)\right| & \lesssim \int_{\mathbb{T}}\left|K\left(t, w_{1}, \tau\right)-K\left(t, w_{2}, \tau\right)\right||d \tau| \\
& \lesssim\left|w_{1}-w_{2}\right| \int_{\mathbb{T}} \sup _{w \in \mathbb{T}}\left|\partial_{w} K(t, w, \tau)\right||d \tau| .
\end{aligned}
$$

Observe that $K(t, w, \tau)$ can be rewritten in the integral form

$$
K(t, w, \tau)=\int_{0}^{t} \partial_{s} g(s, w, \tau) d s, \quad \text { with } \quad g(t, w, \tau) \triangleq \frac{1}{\left|\phi_{j}(w)-\phi_{i}(\tau)+t h_{j}(w)\right|^{\alpha}} .
$$

Thus,

$$
\left|\partial_{w} K(t, w, \tau)\right| \leq \int_{0}^{t}\left|\partial_{w} \partial_{s} g(s, w, \tau)\right| d s .
$$

In view of the formula (3.1) we readily obtain

$$
\begin{aligned}
\partial_{w} g(t, w, \tau)=\frac{-\alpha}{2}[ & \left(\phi_{j}^{\prime}(w)+t h_{j}^{\prime}(w)\right) \frac{\overline{\phi_{j}(w)}-\overline{\phi_{i}(\tau)}+t \overline{h_{j}(w)}}{\left.\mid \phi_{j}(w)-\phi_{i}(\tau)+t h_{j}(w)\right)\left.\right|^{2+\alpha}} \\
& \left.-\frac{\overline{\phi_{j}^{\prime}(w)}+t \overline{h_{j}^{\prime}(w)}}{w^{2}} \frac{\left.\left(\phi_{j}(w)-\phi_{i}(\tau)+t h_{j}(w)\right)\right)}{\left.\mid \phi_{j}(w)-\phi_{i}(\tau)+t h_{j}(w)\right)\left.\right|^{2+\alpha}}\right] .
\end{aligned}
$$

Using straightforward computations combined with the inequality (4.5) yield for any $s \in$ $[0, t]$

$$
\left|\partial_{s} \partial_{w} g(s, w, \tau)\right| \leq C
$$

Hence we get,

$$
\left|\partial_{w} K(t, w, \tau)\right| \leq C|t|
$$

This implies according to the estimate (4.17) that

$$
\left|T_{i j}\left(t, w_{1}\right)-T_{i j}\left(t, w_{2}\right)\right| \leq C|t|\left|w_{1}-w_{2}\right|
$$

and consequently,

$$
\lim _{t \rightarrow 0}\left\|T_{i j}(t, \cdot)\right\|_{C^{1-\alpha}(\mathbb{T})}=0 .
$$

This concludes the desired result.

The next task is to show that the Gâteaux derivatives are continuous operators from the neighborhood $V$ into the Banach space $\mathcal{L}\left(C_{a r}^{1-\alpha}(\mathbb{T}), H\right)$. From the identities (4.11) and (4.16) and since $C^{1-\alpha}(\mathbb{T})$ is an algebra the problem amounts to showing the continuity of the terms $S\left(\phi_{i}, \phi_{j}\right), A_{i}\left(\phi_{j}\right), B_{i}\left(\phi_{j}\right), C_{i}\left(\phi_{j}, h_{i}\right), D_{i}\left(\phi_{j}, h_{i}\right)$ and $E_{i}\left(\phi_{j}, h_{i}\right)$. We shall present here the complete details for the term $S\left(\phi_{i}, \phi_{j}\right)$, with $i \neq j$ and the other terms can be dealt via straightforward variations. Set

$$
\phi_{1}=\mathrm{Id}+f_{1}, \quad \psi_{1}=\mathrm{Id}+g_{1}, \quad \underset{25}{\phi_{2}}=b \mathrm{Id}+f_{2}, \quad \psi_{2}=b \mathrm{Id}+g_{2},
$$


with $\left(f_{1}, f_{2}\right)$ and $\left(g_{1}, g_{2}\right) \in V$. We shall prove the estimate

$$
\left\|S\left(\phi_{i}, \phi_{j}\right)-S\left(\psi_{i}, \psi_{j}\right)\right\|_{C^{1-\alpha}} \leq C\left(\left\|f_{1}-g_{1}\right\|_{C^{2-\alpha}(\mathbb{T})}+\left\|f_{2}-g_{2}\right\|_{C^{2-\alpha}(\mathbb{T})}\right) .
$$

In view of (4.2) we may write

$$
\begin{aligned}
S\left(\phi_{i}, \phi_{j}\right)(w)-S\left(\psi_{i}, \psi_{j}\right)(w) & =f_{\mathbb{T}}\left(\frac{\phi_{i}^{\prime}(\tau)}{\left|\phi_{j}(w)-\phi_{i}(\tau)\right|^{\alpha}}-\frac{\psi_{i}^{\prime}(\tau)}{\left|\psi_{j}(w)-\psi_{i}(\tau)\right|^{\alpha}}\right) d \tau \\
& =f_{\mathbb{T}} \tilde{K}(w, \tau) \psi_{i}^{\prime}(\tau) d \tau+f_{\mathbb{T}} \frac{\phi_{i}^{\prime}(\tau)-\psi_{i}^{\prime}(\tau)}{\left|\phi_{j}(w)-\phi_{i}(\tau)\right|^{\alpha}} d \tau
\end{aligned}
$$

with

$$
\tilde{K}(w, \tau) \triangleq \frac{1}{\left|\phi_{j}(w)-\phi_{i}(\tau)\right|^{\alpha}}-\frac{1}{\left|\psi_{j}(w)-\psi_{i}(\tau)\right|^{\alpha}} .
$$

The estimate of the last term in (4.18) follows immediately from (4.4), that is,

$$
\begin{aligned}
& \left\|f_{\mathbb{T}} \frac{\phi_{i}^{\prime}(\tau)-\psi_{i}^{\prime}(\tau)}{\phi_{j}(\cdot)-\left.\phi_{i}(\tau)\right|^{\alpha}} d \tau\right\|_{C^{1-\alpha}(\mathbb{T})} \leq C\left\|f_{i}^{\prime}-g_{i}^{\prime}\right\|_{L^{\infty}} \\
& \leq C\left\|f_{i}-g_{i}\right\|_{C^{2-\alpha}(\mathbb{T})} .
\end{aligned}
$$

To control the remaining term we introduce the functional

$$
L(w) \triangleq f_{\mathbb{T}} \tilde{K}(w, \tau) \psi^{\prime}(\tau) d \tau .
$$

Owing to the inequalities (4.5) and (4.6) one has

$$
\begin{aligned}
|L(w)| & \lesssim\left\|\phi_{i}\right\|_{\operatorname{Lip}(\mathbb{T})} \int_{\mathbb{T}} \frac{|| \psi_{j}(w)-\left.\psi_{i}(\tau)\right|^{\alpha}-\left|\phi_{j}(w)-\phi_{i}(\tau)\right|^{\alpha} \mid}{\left|\phi_{j}(w)-\phi_{i}(\tau)\right|^{\alpha}\left|\psi_{j}(w)-\psi_{i}(\tau)\right|^{\alpha}}|d \tau| \\
& \lesssim\left\|\psi_{j}-\phi_{j}\right\|_{L^{\infty}(\mathbb{T})} \\
& \lesssim\left\|f_{j}-f_{j}\right\|_{L^{\infty}(\mathbb{T})} .
\end{aligned}
$$

Now let $w_{1} \neq w_{2} \in \mathbb{T}$, then we have

$$
\begin{aligned}
\left|L\left(w_{1}\right)-L\left(w_{2}\right)\right| & \lesssim \int_{\mathbb{T}}\left|\tilde{K}\left(w_{1}, \tau\right)-\tilde{K}\left(w_{2}, \tau\right)\right||d \tau| \\
& \lesssim\left|w_{1}-w_{2}\right| \int_{\mathbb{T}} \sup _{w \in \mathbb{T}}\left|\partial_{w} \tilde{K}(w, \tau)\right||d \tau| .
\end{aligned}
$$

In view of (3.1) the derivative of $K(w, \tau)$ with respect to $w$ is given by

$$
\partial_{w} \tilde{K}(w, \tau)=-\frac{\alpha}{2}\left(\overline{\mathcal{I}(w, \tau)}-\frac{\mathcal{I}(w, \tau)}{w^{2}}\right),
$$

where

$$
\mathcal{I}(w, \tau) \triangleq \overline{\phi_{j}^{\prime}(w)} \frac{\phi_{j}(w)-\phi_{i}(\tau)}{\left|\phi_{j}(w)-\phi_{i}(\tau)\right|^{\alpha+2}}-\overline{\psi_{j}^{\prime}(w)} \frac{\psi_{j}(w)-\psi_{i}(\tau)}{\left|\psi_{j}(w)-\psi_{i}(\tau)\right|^{\alpha+2}} .
$$

We shall transform this quantity into,

$$
\mathcal{I}(w, \tau)=\mathcal{I}_{1}(w, \tau)+\mathcal{I}_{26}(w, \tau)+\mathcal{I}_{3}(w, \tau),
$$


with

$$
\begin{gathered}
\mathcal{I}_{1}(w, \tau) \triangleq \overline{\phi_{j}^{\prime}(w)} \frac{\left(\phi_{j}-\psi_{j}\right)(w)-\left(\phi_{i}-\psi_{i}\right)(\tau)}{\left|\phi_{j}(w)-\phi_{i}(\tau)\right|^{\alpha+2}} \\
\mathcal{I}_{2}(w, \tau) \triangleq\left(\overline{\phi_{j}^{\prime}(w)}-\overline{\psi_{j}^{\prime}(w)}\right) \frac{\psi_{j}(w)-\psi_{i}(\tau)}{\left|\psi_{j}(w)-\psi_{i}(\tau)\right|^{\alpha+2}}
\end{gathered}
$$

and

$$
\mathcal{I}_{3}(w, \tau) \triangleq \overline{\phi_{j}^{\prime}(w)}\left(\psi_{i}(\tau)-\psi_{j}(w)\right) \frac{\left|\phi_{j}(w)-\phi_{i}(\tau)\right|^{\alpha+2}-\left|\psi_{j}(w)-\psi_{i}(\tau)\right|^{\alpha+2}}{\left|\phi_{j}(w)-\phi_{i}(\tau)\right|^{\alpha+2}\left|\psi_{j}(w)-\psi_{i}(\tau)\right|^{\alpha+2}} .
$$

For the first and the second terms one readily gets by Sobolev embeddings

$$
\left|\mathcal{I}_{1}(w, \tau)\right|+\left|\mathcal{I}_{2}(w, \tau)\right| \lesssim\left\|\phi_{i}-\psi_{i}\right\|_{C^{2-\alpha}(\mathbb{T})}+\left\|\phi_{j}-\psi_{j}\right\|_{C^{2-\alpha}(\mathbb{T})} .
$$

To estimate the last term we shall use the inequality (4.15) combined with the estimate (4.5),

$$
\frac{|| \phi_{j}(w)-\left.\phi_{i}(\tau)\right|^{\alpha+2}-\left|\psi_{j}(w)-\psi_{i}(\tau)\right|^{\alpha+2} \mid}{\left|\phi_{j}(w)-\phi_{i}(\tau)\right|^{\alpha+2}\left|\psi_{j}(w)-\psi_{i}(\tau)\right|^{\alpha+2}} \lesssim\left\|\phi_{j}-\psi_{j}\right\|_{L^{\infty}(\mathbb{T})}
$$

and consequently,

$$
\left|\mathcal{I}_{3}(w, \tau)\right| \lesssim\left\|\phi_{j}-\psi_{j}\right\|_{C^{2-\alpha}(\mathbb{T})} .
$$

Putting together (4.22) and (4.23) we find,

$$
|\mathcal{I}(w, \tau)| \lesssim\left\|\phi_{1}-\psi_{1}\right\|_{C^{2-\alpha}(\mathbb{T})}+\left\|\phi_{2}-\psi_{2}\right\|_{C^{2-\alpha}(\mathbb{T})}
$$

Therefore

$$
\left|\partial_{w} \tilde{K}(w, \tau)\right| \leq C\left(f_{1}-g_{1}\left\|_{C^{2-\alpha}(\mathbb{T})}+\right\| f_{2}-g_{2} \|_{C^{2-\alpha}(\mathbb{T})}\right) .
$$

Inserting this inequality into the estimate (4.21) we get

$$
\left|L\left(w_{1}\right)-L\left(w_{2}\right)\right| \leq C\left(f_{1}-g_{1}\left\|_{C^{2-\alpha}(\mathbb{T})}+\right\| f_{2}-g_{2} \|_{C^{2-\alpha}(\mathbb{T})}\right)\left|w_{1}-w_{2}\right|^{1-\alpha} .
$$

Putting together the last estimate with the estimate (4.19), we obtain

$$
\left\|S\left(\phi_{i}, \phi_{j}\right)-S\left(\psi_{i}, \psi_{j}\right)\right\|_{C^{1-\alpha}(\mathbb{T})} \lesssim\left\|f_{1}-g_{1}\right\|_{C^{2-\alpha}(\mathbb{T})}+\left\|f_{2}-g_{2}\right\|_{C^{2-\alpha}(\mathbb{T})} .
$$

This concludes the proof of the Proposition 4.1.

\section{Spectral Study}

The main goal of this section is to perform a spectral study of the linearized operator of $G$ at the annular solution $(\Omega, 0,0)$ and denoted by the differential $D G(\Omega, 0,0)$. In particular, we shall identify the values $\Omega$ for which the kernel of $D G(\Omega, 0,0)$ is not trivial leading to what we call the dispersion relation. Therefore the next step is to look among the "nonlinear eigenvalues" $\Omega$ those corresponding to one-dimensional kernels which is an important assumption in Crandall-Rabinowitz's Theorem. This task is very complicate compared to the previous cases discussed in $[6,20,23]$. This is due to the multiple parameters $\alpha, b$ and $m$ in this problem and especially to the nonlinear and implicit structure of the coefficients appearing in the dispersion relation. We will be able to validate only a sufficient, but still a satisfactory result compared to Euler equation, with a restriction on the symmetry of the V-states. This will be deeply discussed in the Subsection 5.3 devoted to the monotonicity of the eigenvalues. 
5.1. Linearized operator. We propose to compute explicitly the differential $D G(\Omega, 0,0)$ and show that it acts as a Fourier multiplier. Since $G=\left(G_{1}, G_{2}\right)$ then for given $\left(h_{1}, h_{2}\right) \in X$, we have

$$
D G(\Omega, 0,0)\left(h_{1}, h_{2}\right)=\left(\begin{array}{c}
D_{f_{1}} G_{1}(\Omega, 0,0) h_{1}+D_{f_{2}} G_{1}(\Omega, 0,0) h_{2} \\
D_{f_{1}} G_{2}(\Omega, 0,0) h_{1}+D_{f_{2}} G_{2}(\Omega, 0,0) h_{2}
\end{array}\right) .
$$

where we recall the function spaces

$$
X=C_{a r}^{2-\alpha}(\mathbb{T}) \times C_{a r}^{2-\alpha}(\mathbb{T})
$$

and

$$
C_{a r}^{2-\alpha}(\mathbb{T})=\left\{f \in C^{2-\alpha}(\mathbb{T}) ; f(w)=\sum_{n \geq 1} a_{n} \bar{w}^{n}, w \in \mathbb{T}, a_{n} \in \mathbb{R}, n \in \mathbb{N}^{\star}\right\} .
$$

Putting together the formulas (4.3), (4.11) and (4.16) with $j=1$ and $j=2$, where we replace $\phi_{1}$ by Id and $\phi_{2}$ by $b$ Id we get

$$
\begin{aligned}
& D G_{1}(\Omega, 0,0) h(w)=\Omega \mathcal{L}_{0}\left(h_{1}\right)(w)-C_{\alpha} \mathcal{L}_{1}\left(h_{1}\right)(w)+C_{\alpha} \mathcal{L}_{2}\left(h_{1}, h_{2}\right)(w), \\
& D G_{2}(\Omega, 0,0) h(w)=b\left(\Omega \mathcal{L}_{0}\left(h_{2}\right)(w)+b^{-\alpha} C_{\alpha} \mathcal{L}_{1}\left(h_{2}\right)(w)-C_{\alpha} \mathcal{L}_{3}\left(h_{1}, h_{2}\right)(w)\right),
\end{aligned}
$$

with

$$
\begin{aligned}
& \mathcal{L}_{0}\left(h_{j}\right)(w) \triangleq \operatorname{Im}\left\{\overline{h_{j}^{\prime}(w)}+\bar{w} h_{j}(w)\right\} \\
& \mathcal{L}_{1}\left(h_{j}\right)(w) \triangleq \operatorname{Im}\left\{\bar{w} \overline{h_{j}^{\prime}(w)} f_{\mathbb{T}} \frac{d \tau}{|w-\tau|^{\alpha}}+\bar{w} f_{\mathbb{T}} \frac{h_{j}^{\prime}(\tau)}{|w-\tau|^{\alpha}} d \tau\right. \\
& \left.-\alpha \bar{w} f_{\mathbb{T}} \frac{\operatorname{Re}\left[(w-\tau)\left(\overline{h_{j}(w)}-\overline{h_{j}(\tau)}\right)\right]}{|w-\tau|^{\alpha+2}} d \tau\right\}, \\
& \mathcal{L}_{2}\left(h_{1}, h_{2}\right)(w) \triangleq \operatorname{Im}\left\{b \bar{w} \overline{h_{1}^{\prime}(w)} f_{\mathbb{T}} \frac{d \tau}{|w-b \tau|^{\alpha}}+\bar{w} f_{\mathbb{T}} \frac{h_{2}^{\prime}(\tau) d \tau}{|w-b \tau|^{\alpha}}\right. \\
& \left.-\alpha b \bar{w} f_{\mathbb{T}} \frac{\operatorname{Re}\left[(w-b \tau)\left(\overline{h_{1}(w)}-\overline{h_{2}(\tau)}\right)\right] d \tau}{|w-b \tau|^{\alpha+2}}\right\},
\end{aligned}
$$

and

$$
\begin{aligned}
\mathcal{L}_{3}\left(h_{1}, h_{2}\right)(w) \triangleq \operatorname{Im} & \left\{b \bar{w} \overline{h_{2}^{\prime}(w)} f_{\mathbb{T}} \frac{d \tau}{|b w-\tau|^{\alpha}}+\bar{w} f_{\mathbb{T}} \frac{h_{1}^{\prime}(\tau)}{|b w-\tau|^{\alpha}} d \tau\right. \\
& \left.-\alpha \bar{w} f_{\mathbb{T}} \frac{\operatorname{Re}\left[(b w-\tau)\left(\overline{h_{2}(w)}-\overline{h_{1}(\tau)}\right)\right]}{|b w-\tau|^{\alpha+2}} d \tau\right\} .
\end{aligned}
$$

We shall now compute the Fourier series of the mapping $w \mapsto D G(\Omega, 0,0)\left(h_{1}, h_{2}\right)(w)$ with

$$
h_{1}(w)=\sum_{n=1}^{\infty} a_{n} \bar{w}^{n} \quad \text { and } \quad h_{2}(w)=\sum_{n=1}^{\infty} c_{n} \bar{w}^{n}, \quad w \in \mathbb{T},
$$

where $a_{n}$ and $c_{n}$ are real for all $n \in \mathbb{N}^{\star}$. This is summarized in the following lemma. 
Lemma 5.1. Let $\alpha \in(0,1)$ and $b \in(0,1)$. We set

$$
\Lambda_{n}(b) \triangleq \frac{1}{b} \int_{0}^{+\infty} \frac{J_{n}(b t) J_{n}(t)}{t^{1-\alpha}} d t
$$

and

$$
\Theta_{n} \triangleq \Lambda_{1}(1)-\Lambda_{n}(1)
$$

where $J_{n}$ refers to the Bessel function of the first kind. Then, we have

$$
D G(\Omega, 0,0)\left(h_{1}, h_{2}\right)(w)=\frac{i}{2} \sum_{n \geq 1}(n+1) M_{n+1}^{\alpha}\left(\begin{array}{c}
a_{n} \\
c_{n}
\end{array}\right)\left(w^{n+1}-\bar{w}^{n+1}\right) .
$$

where the matrix $M_{n}$ is given for $n \geq 2$ by

$$
M_{n}^{\alpha} \triangleq\left(\begin{array}{cc}
\Omega-\Theta_{n}+b^{2} \Lambda_{1}(b) & -b^{2} \Lambda_{n}(b) \\
b \Lambda_{n}(b) & b \Omega+b^{1-\alpha} \Theta_{n}-b \Lambda_{1}(b)
\end{array}\right) .
$$

The determinant of this matrix is given by

$$
\operatorname{det}\left(M_{n}^{\alpha}\right)=\left(\Omega-\Theta_{n}+b^{2} \Lambda_{1}(b)\right)\left(b \Omega+b^{1-\alpha} \Theta_{n}-b \Lambda_{1}(b)\right)+b^{3} \Lambda_{n}^{2}(b) .
$$

Proof. First we shall compute $D G_{1}(\Omega, 0,0)\left(h_{1}, h_{2}\right)$. For this goal we start with calculating the term $\mathcal{L}_{0}\left(h_{1}(w)\right)$ of the right-hand side of (5.1) which is easy compared to the other terms. Thus by straightforward computations we obtain

$$
\begin{aligned}
\mathcal{L}_{0}\left(h_{1}\right)(w) & =\operatorname{Im}\left\{\sum_{n \geq 1}\left(a_{n} \bar{w}^{n+1}-n a_{n} w^{n+1}\right)\right\} \\
& =\frac{i}{2} \sum_{n \geq 1}(n+1) a_{n}\left(w^{n+1}-\bar{w}^{n+1}\right) .
\end{aligned}
$$

The computation of the second term $\mathcal{L}_{1}\left(h_{1}\right)(w)$ was done in the paper [20] dealing with the simply connected domain. It is given by

$$
C_{\alpha} \mathcal{L}_{1}\left(h_{1}\right)(w)=\frac{i}{2} \sum_{n \geq 1} a_{n}(n+1) \frac{\Gamma(1-\alpha)}{2^{1-\alpha} \Gamma^{2}\left(1-\frac{\alpha}{2}\right)}\left(\frac{\Gamma\left(1+\frac{\alpha}{2}\right)}{\Gamma\left(2-\frac{\alpha}{2}\right)}-\frac{\Gamma\left(n+1+\frac{\alpha}{2}\right)}{\Gamma\left(n+2-\frac{\alpha}{2}\right)}\right)\left(w^{n+1}-\bar{w}^{n+1}\right) .
$$

We shall later establish the identity (5.26) which gives here

$$
C_{\alpha} \mathcal{L}_{1}\left(h_{1}\right)(w)=\frac{i}{2} \sum_{n \geq 1} a_{n}(n+1) \Theta_{n+1}\left(w^{n+1}-\bar{w}^{n+1}\right) .
$$

To compute the term $\mathcal{L}_{2}\left(h_{1}, h_{2}\right)(w)$ we first split it into two parts as follows,

$$
\mathcal{L}_{2}\left(h_{1}, h_{2}\right)(w)=\operatorname{Im}\left\{\mathrm{I}_{1}(w)+\mathrm{I}_{2}(w)\right\},
$$

with

and

$$
\mathrm{I}_{1}(w) \triangleq \frac{b \overline{h_{1}^{\prime}(w)}}{w} f_{\mathbb{T}} \frac{d \tau}{|w-b \tau|^{\alpha}}-\frac{\alpha b}{2 w} f_{\mathbb{T}} \frac{(w-b \tau)\left(\overline{h_{1}(w)}-\overline{h_{2}(\tau)}\right)}{|w-b \tau|^{\alpha+2}} d \tau
$$

$$
\mathrm{I}_{2}(w) \triangleq \frac{1}{w} f_{\mathbb{T}} \frac{h_{2}^{\prime}(\tau)}{|w-b \tau|^{\alpha}} d \tau-\frac{\alpha b}{2 w} f_{29} \frac{(\bar{w}-b \bar{\tau})\left(h_{1}(w)-h_{2}(\tau)\right)}{|w-b \tau|^{\alpha+2}} d \tau .
$$


By using the Fourier expansions of $h_{1}$ and $h_{2}$ we get,

$$
\mathrm{I}_{1}(w)=-b \sum_{n \geq 1} n a_{n} w^{n} f_{\mathbb{T}} \frac{d \tau}{|w-b \tau|^{\alpha}}-\frac{\alpha}{2} b \bar{w} \sum_{n \geq 1} f_{\mathbb{T}} \frac{(w-b \tau)\left(a_{n} w^{n}-c_{n} \tau^{n}\right)}{|w-b \tau|^{\alpha+2}} d \tau .
$$

Then by applying the formula (3.19) with $n=1$ to the first term and the formula (3.20) to the second term we find

$$
\begin{aligned}
\mathrm{I}_{1}(w)=-\frac{\alpha}{2} b^{2} \sum_{n \geq 1} n a_{n} w^{n+1} F\left(\frac{\alpha}{2}, 1+\frac{\alpha}{2} ; 2 ; b^{2}\right) \\
-\frac{\alpha}{2} b^{2} \sum_{n \geq 0} w^{n+1}\left[a_{n}\left(1+\frac{\alpha}{2}\right) F\left(\frac{\alpha}{2}, 2+\frac{\alpha}{2} ; 2 ; b^{2}\right)\right. \\
\left.\quad-c_{n} b^{n} \frac{\left(1+\frac{\alpha}{2}\right)_{n+1}}{(n+1) !} F\left(\frac{\alpha}{2}, n+2+\frac{\alpha}{2} ; n+2 ; b^{2}\right)\right] \\
\triangleq-\frac{\alpha}{2} b^{2} \sum_{n \geq 0}\left(a_{n} \gamma_{n}+c_{n} \delta_{n}\right) w^{n+1},
\end{aligned}
$$

where we have used in the last equality the notation,

$$
\gamma_{n} \triangleq\left(1+\frac{\alpha}{2}\right) F\left(\frac{\alpha}{2}, 2+\frac{\alpha}{2} ; 2 ; b^{2}\right)+n F\left(\frac{\alpha}{2}, 1+\frac{\alpha}{2} ; 2 ; b^{2}\right)
$$

and

$$
\delta_{n}=-b^{n} \frac{\left(1+\frac{\alpha}{2}\right)_{n+1}}{(n+1) !} F\left(\frac{\alpha}{2}, n+2+\frac{\alpha}{2} ; n+2 ; b^{2}\right) .
$$

Similarly, the second term $\mathrm{I}_{2}(w)$ may be written in the form,

$$
\mathrm{I}_{2}(w)=-\bar{w} \sum_{n \geq 0} n c_{n} f_{\mathbb{T}} \frac{\bar{\tau}^{n+1} d \tau}{|w-b|^{\alpha}}-\frac{\alpha}{2} b \bar{w} \sum_{n \geq 0} f_{\mathbb{T}} \frac{(\bar{w}-b \bar{\tau})\left(a_{n} \bar{w}^{n}-c_{n} \bar{\tau}^{n}\right)}{|w-b \tau|^{\alpha+2}} d \tau .
$$

Using the elementary fact

$$
f_{\mathbb{T}} \frac{\bar{\tau}^{n+1} d \tau}{|w-b \tau|^{\alpha}}=\overline{f_{\mathbb{T}} \frac{\tau^{n-1} d \tau}{|w-b \tau|^{\alpha}}}
$$

combined with the formulas (3.19) and (3.21) we obtain

$$
\begin{aligned}
& \mathrm{I}_{2}(w)=-\sum_{n \geq 1} n c_{n} \frac{\left(\frac{\alpha}{2}\right)_{n}}{n !} \bar{w}^{n+1} b^{n} F\left(\frac{\alpha}{2}, n+\frac{\alpha}{2} ; n+1 ; b^{2}\right) \\
& -\frac{\alpha}{2} \sum_{n \geq 1} \bar{w}^{n+1}\left[a_{n} b^{2} \frac{\alpha}{2} F\left(\frac{\alpha}{2}+1, \frac{\alpha}{2}+1 ; 2 ; b^{2}\right)-c_{n} b^{n} \frac{\left(1+\frac{\alpha}{2}\right)_{n-1}}{(n-1) !} F\left(\frac{\alpha}{2}, n+\frac{\alpha}{2} ; n ; b^{2}\right)\right] \\
& =-\frac{\alpha}{2} \sum_{n \geq 1} \bar{w}^{n+1}\left[a_{n} b^{2} \frac{\alpha}{2} F\left(\frac{\alpha}{2}+1, \frac{\alpha}{2}+1 ; 2 ; b^{2}\right)\right. \\
& \left.+c_{n} \frac{\left(1+\frac{\alpha}{2}\right)_{n-1}}{(n-1) !} b^{n}\left(F\left(\frac{\alpha}{2}, n+\frac{\alpha}{2} ; n+1 ; b^{2}\right)-F\left(\frac{\alpha}{2}, n+\frac{\alpha}{2} ; n ; b^{2}\right)\right)\right] .
\end{aligned}
$$


Thus owing to the formula (3.11) applied with $a=\frac{\alpha}{2}, b=\frac{\alpha}{2}+n$ and $c=n$ one gets

$$
\mathrm{I}_{2}(w)=-\frac{\alpha}{2} b^{2} \sum_{n \geq 1}\left(\alpha_{n} a_{n}+\beta_{n} c_{n}\right) \bar{w}^{n+1},
$$

where $\alpha_{n}$ and $\beta_{n}$ are defined by,

$$
\alpha_{n} \triangleq \frac{\alpha}{2} F\left(\frac{\alpha}{2}+1, \frac{\alpha}{2}+1 ; 2 ; b^{2}\right)
$$

and

$$
\beta_{n} \triangleq-\frac{\left(\frac{\alpha}{2}\right)_{n+1}}{(n+1) !} b^{n} F\left(\frac{\alpha}{2}+1, n+1+\frac{\alpha}{2} ; n+2 ; b^{2}\right) .
$$

Inserting the identities (5.13) and (5.10) into (5.9) we find

$$
\begin{aligned}
\mathcal{L}_{2}\left(h_{1}, h_{2}\right)(w) & =-\frac{\alpha}{2} b^{2} \operatorname{Im}\left\{\sum_{n \geq 1}\left(a_{n} \gamma_{n}+c_{n} \delta_{n}\right) w^{n+1}+\sum_{n \geq 1}\left(a_{n} \alpha_{n}+c_{n} \beta_{n}\right) \bar{w}^{n+1}\right\} \\
& =i \frac{\alpha}{4} b^{2} \sum_{n \geq 1}\left(w^{n+1}-\bar{w}^{n+1}\right)\left[a_{n}\left(\gamma_{n}-\alpha_{n}\right)+c_{n}\left(\delta_{n}-\beta_{n}\right)\right] .
\end{aligned}
$$

To compute $\gamma_{n}-\alpha_{n}$ we shall use the formula (3.16) which gives

$$
\begin{aligned}
\gamma_{n}-\alpha_{n} & =\left(1+\frac{\alpha}{2}\right) F\left(\frac{\alpha}{2}, 2+\frac{\alpha}{2} ; 2 ; b^{2}\right)-\frac{\alpha}{2} F\left(\frac{\alpha}{2}+1, \frac{\alpha}{2}+1 ; 2 ; b^{2}\right)+n F\left(\frac{\alpha}{2}, 1+\frac{\alpha}{2} ; 2 ; b^{2}\right) \\
& =(n+1) F\left(\frac{\alpha}{2}, 1+\frac{\alpha}{2} ; 2 ; b^{2}\right) .
\end{aligned}
$$

Similarly we have

$$
\begin{aligned}
\delta_{n}-\beta_{n}=-b^{n} \frac{\left(1+\frac{\alpha}{2}\right)_{n}}{(n+1) !}[ & \left(n+1+\frac{\alpha}{2}\right) F\left(\frac{\alpha}{2}, n+2+\frac{\alpha}{2} ; n+2 ; b^{2}\right) \\
& \left.-\frac{\alpha}{2} F\left(\frac{\alpha}{2}+1, n+1+\frac{\alpha}{2} ; n+2 ; b^{2}\right)\right]
\end{aligned}
$$

and therefore using once again the identity (3.16) we find

$$
\delta_{n}-\beta_{n}=-b^{n} \frac{\left(1+\frac{\alpha}{2}\right)_{n}}{n !} F\left(\frac{\alpha}{2}, n+1+\frac{\alpha}{2}, n+2, b^{2}\right) .
$$

Consequently the Fourier expansion of $\mathcal{L}_{2}\left(h_{1}, h_{2}\right)$ is described by the formula

$$
\begin{aligned}
\mathcal{L}_{2}\left(h_{1}, h_{2}\right)(w)=\frac{i}{2} b^{2} \sum_{n \geq 1}(n+1) & {\left[a_{n} \frac{\alpha}{2} F\left(\frac{\alpha}{2}, 1+\frac{\alpha}{2}, 2, b^{2}\right)\right.} \\
& \left.-c_{n} b^{n} \frac{\left(\frac{\alpha}{2}\right)_{n+1}}{(n+1) !} F\left(\frac{\alpha}{2}, n+1+\frac{\alpha}{2}, n+2, b^{2}\right)\right]\left(w^{n+1}-\bar{w}^{n+1}\right) .
\end{aligned}
$$

By virtue of the identity (5.24) we get

$$
C_{\alpha} \mathcal{L}_{2}\left(h_{1}, h_{2}\right)(w)=\frac{i}{2} \sum_{n \geq 1}(n+1)\left[a_{n} b^{2} \Lambda_{1}(b)-c_{n} b^{2} \Lambda_{n+1}(b)\right]\left(w^{n+1}-\bar{w}^{n+1}\right) .
$$

Finally inserting (5.7), (5.8) and (5.14) into (5.1) we find 


$$
\begin{aligned}
D G_{1}(\Omega, 0,0)\left(h_{1}, h_{2}\right)(w)=\frac{i}{2} \sum_{n \geq 1} & (n+1)\left[a_{n}\left(\Omega-\Theta_{n+1}+b^{2} \Lambda_{1}(b)\right)-c_{n} b^{2} \Lambda_{n+1}(b)\right] \\
\times & \times\left(w^{n+1}-\bar{w}^{n+1}\right) .
\end{aligned}
$$

Next, we shall move to the computations of $D G_{2}(\Omega, 0,0)\left(h_{1}, h_{2}\right)$ defined in (5.2). The first two terms are done in the preceding step and therefore it remains just to compute the term $\mathcal{L}_{3}\left(h_{1}, h_{2}\right)$. It may be splitted into two terms,

$$
\mathcal{L}_{3}\left(h_{1}, h_{2}\right)(w)=\operatorname{Im}\left\{\tilde{\mathrm{I}}_{1}(w)+\tilde{\mathrm{I}}_{2}(w)\right\},
$$

with

and

$$
\tilde{\mathrm{I}}_{1}(w) \triangleq \frac{\overline{h_{2}^{\prime}(w)}}{b w} f_{\mathbb{T}} \frac{d \tau}{|b w-\tau|^{\alpha}}-\frac{\alpha}{2 w} f_{\mathbb{T}} \frac{(b w-\tau)\left(\overline{h_{2}(w)}-\overline{h_{1}(\tau)}\right)}{|b w-\tau|^{\alpha+2}} d \tau
$$

$$
\tilde{\mathrm{I}}_{2}(w) \triangleq \frac{1}{w} f_{\mathbb{T}} \frac{h_{1}^{\prime}(\tau) d \tau}{|b w-\tau|^{\alpha}}-\frac{\alpha}{2 w} f_{\mathbb{T}} \frac{(b \bar{w}-\bar{\tau})\left(h_{2}(w)-h_{1}(\tau)\right)}{|b w-\tau|^{\alpha+2}} d \tau .
$$

To compute the first term $\tilde{\mathrm{I}}_{1}(w)$ we write

$$
\tilde{\mathrm{I}}_{1}(w)=-\sum_{n \geq 1} n \frac{c_{n}}{b} w^{n} f_{\mathbb{T}} \frac{d \tau}{|b w-\tau|^{\alpha}}-\frac{\alpha}{2 w} \sum_{n \geq 1} f_{\mathbb{T}} \frac{(b w-\tau)\left(c_{n} w^{n}-a_{n} \tau^{n}\right)}{|b w-\tau|^{\alpha+2}} d \tau .
$$

Thus applying successively the formula (3.19) to the first term with $n=1$ and the formula (3.22) to the second one we get

$$
\begin{aligned}
\tilde{\mathrm{I}}_{1}(w)= & -\frac{\alpha}{2} \sum_{n \geq 1} n c_{n} w^{n+1} F\left(\frac{\alpha}{2}, 1+\frac{\alpha}{2} ; 2 ; b^{2}\right) \\
& +\frac{\alpha}{2} \sum_{n \geq 1} w^{n+1}\left[c_{n} b^{2} \frac{\alpha}{4}\left(1+\frac{\alpha}{2}\right) F\left(1+\frac{\alpha}{2}, 2+\frac{\alpha}{2} ; 3 ; b^{2}\right)\right. \\
& \left.\quad-a_{n} b^{n+2} \frac{\left(\frac{\alpha}{2}\right)_{n+2}}{(n+2) !} F\left(1+\frac{\alpha}{2}, n+2+\frac{\alpha}{2} ; n+3 ; b^{2}\right)\right] \\
= & -\frac{\alpha}{2} \sum_{n \geq 1}\left(a_{n} \tilde{\gamma}_{n}+c_{n} \tilde{\delta}_{n}\right) w^{n+1}
\end{aligned}
$$

with

$$
\tilde{\gamma}_{n} \triangleq b^{n+2} \frac{\left(\frac{\alpha}{2}\right)_{n+2}}{(n+2) !} F\left(1+\frac{\alpha}{2}, n+2+\frac{\alpha}{2} ; n+3 ; b^{2}\right)
$$

and

$$
\tilde{\delta}_{n} \triangleq n F\left(\frac{\alpha}{2}, 1+\frac{\alpha}{2} ; 2 ; b^{2}\right)-b^{2} \frac{\alpha}{4}\left(1+\frac{\alpha}{2}\right) F\left(1+\frac{\alpha}{2}, 2+\frac{\alpha}{2} ; 3 ; b^{2}\right) .
$$

As to the term $\tilde{\mathrm{I}}_{2}(w)$ we write

$$
\tilde{\mathrm{I}}_{2}(w)=-\frac{1}{w} \sum_{n \geq 1} n a_{n} f_{\mathbb{T}} \frac{\bar{\tau}^{n+1} d \tau}{|b w-\tau|^{\alpha}}-\frac{\alpha}{2 w} \sum_{n \geq 1} f_{\mathbb{T}} \frac{(b \bar{w}-\bar{\tau})\left(c_{n} \bar{w}^{n}-a_{n} \bar{\tau}^{n}\right) d \tau}{|b w-\tau|^{\alpha+2}} .
$$


Owing to (5.11) and using the formulae (3.19) and (3.23), one gets

$$
\begin{aligned}
\tilde{\mathrm{I}}_{2}(w)= & -\sum_{n \geq 1} n a_{n} \bar{w}^{n+1} b^{n} \frac{\left(\frac{\alpha}{2}\right)_{n}}{n !} F\left(\frac{\alpha}{2}, n+\frac{\alpha}{2} ; n+1 ; b^{2}\right) \\
& -\frac{\alpha}{2} \sum_{n \geq 1} \bar{w}^{n+1}\left[-c_{n} F\left(\frac{\alpha}{2}, \frac{\alpha}{2}+1 ; 1 ; b^{2}\right)+a_{n} b^{n} \frac{\left(\frac{\alpha}{2}\right)_{n}}{n !} F\left(\frac{\alpha}{2}+1, n+\frac{\alpha}{2} ; n+1 ; b^{2}\right)\right] \\
(5.18)= & -\frac{\alpha}{2} \sum_{n \geq 1}\left(a_{n} \tilde{\alpha}_{n}+c_{n} \tilde{\beta}_{n}\right) \bar{w}^{n+1},
\end{aligned}
$$

with

$$
\tilde{\alpha}_{n} \triangleq \frac{\left(1+\frac{\alpha}{2}\right)_{n-1}}{n !} b^{n}\left[n F\left(\frac{\alpha}{2}, n+\frac{\alpha}{2} ; n+1 ; b^{2}\right)+\frac{\alpha}{2} F\left(1+\frac{\alpha}{2}, n+\frac{\alpha}{2} ; n+1 ; b^{2}\right)\right]
$$

and

$$
\tilde{\beta}_{n} \triangleq-F\left(\frac{\alpha}{2}, \frac{\alpha}{2}+1 ; 1 ; b^{2}\right) .
$$

Now inserting the identities (5.17) and (5.18) into (5.16) we find

$$
\begin{aligned}
\mathcal{L}_{3}\left(h_{1}, h_{2}\right)(w) & =-\frac{\alpha}{2} \operatorname{Im}\left\{\sum_{n \geq 1}\left(a_{n} \tilde{\gamma}_{n}+c_{n} \tilde{\delta}_{n}\right) w^{n+1}+\sum_{n \geq 1}\left(a_{n} \tilde{\alpha}_{n}+c_{n} \tilde{\beta}_{n}\right) \bar{w}^{n+1}\right\} \\
& =i \frac{\alpha}{4} \sum_{n \geq 1}\left[a_{n}\left(\tilde{\gamma}_{n}-\tilde{\alpha}_{n}\right)+c_{n}\left(\tilde{\delta}_{n}-\tilde{\beta}_{n}\right)\right]\left(w^{n+1}-\bar{w}^{n+1}\right) .
\end{aligned}
$$

From the foregoing expressions for $\tilde{\gamma}_{n}$ and $\tilde{\alpha}_{n}$ one may write,

$$
\begin{aligned}
& \tilde{\gamma}_{n}-\tilde{\alpha}_{n}=b^{n+2} \frac{\left(\frac{\alpha}{2}\right)_{n+2}}{(n+2) !} F\left(1+\frac{\alpha}{2}, n+2+\frac{\alpha}{2} ; n+3 ; b^{2}\right) \\
&-\frac{\left(1+\frac{\alpha}{2}\right)_{n-1}}{n !} b^{n} {\left[n F\left(\frac{\alpha}{2}, n+\frac{\alpha}{2} ; n+1 ; b^{2}\right)+\frac{\alpha}{2} F\left(1+\frac{\alpha}{2}, n+\frac{\alpha}{2} ; n+1 ; b^{2}\right)\right] } \\
&=\frac{\left(1+\frac{\alpha}{2}\right)_{n-1}}{n !} b^{n}\left[b^{2} \frac{\frac{\alpha}{2}\left(\frac{\alpha}{2}+n\right)\left(\frac{\alpha}{2}+1+n\right)}{(n+2)(n+1)} F\left(1+\frac{\alpha}{2}, n+2+\frac{\alpha}{2} ; n+3 ; b^{2}\right)\right. \\
&\left.\quad-n F\left(\frac{\alpha}{2}, n+\frac{\alpha}{2} ; n+1 ; b^{2}\right)-\frac{\alpha}{2} F\left(1+\frac{\alpha}{2}, n+\frac{\alpha}{2} ; n+1 ; b^{2}\right)\right] .
\end{aligned}
$$

Hence using the formula (3.11) with $a=\frac{\alpha}{2}, b=n+1+\frac{\alpha}{2}$ and $c=n+1$ yields

$$
\begin{aligned}
\tilde{\gamma}_{n}-\tilde{\alpha}_{n}=\frac{\left(1+\frac{\alpha}{2}\right)_{n-1}}{n !} b^{n}[ & \left(\frac{\alpha}{2}+n\right) F\left(\frac{\alpha}{2}, n+1+\frac{\alpha}{2} ; n+1 ; b^{2}\right) \\
& -\left(\frac{\alpha}{2}+n\right) F\left(\frac{\alpha}{2}, n+1+\frac{\alpha}{2} ; n+2 ; b^{2}\right) \\
& \left.-n F\left(\frac{\alpha}{2}, n+\frac{\alpha}{2} ; n+1 ; b^{2}\right)-\frac{\alpha}{2} F\left(1+\frac{\alpha}{2}, n+\frac{\alpha}{2} ; n+1 ; b^{2}\right)\right] .
\end{aligned}
$$

Applying the formula (3.16) with $a=\frac{\alpha}{2}, b=\frac{\alpha}{2}+n$ and $c=n+1$ we get

$$
\left(\frac{\alpha}{2}+n\right) F\left(\frac{\alpha}{2}, n+1+\frac{\alpha}{2} ; n+1 ; b^{2}\right)-\frac{\alpha}{2} F\left(1+\frac{\alpha}{2}, n+\frac{\alpha}{2} ; n+1 ; b^{2}\right)=n F\left(\frac{\alpha}{2}, n+\frac{\alpha}{2} ; n+1 ; b^{2}\right) .
$$


This implies,

$$
\tilde{\gamma}_{n}-\tilde{\alpha}_{n}=-\frac{\left(1+\frac{\alpha}{2}\right)_{n}}{n !} b^{n} F\left(\frac{\alpha}{2}, n+1+\frac{\alpha}{2} ; n+2 ; b^{2}\right) .
$$

Using the expressions of $\tilde{\delta}_{n}$ and $\tilde{\beta}_{n}$ combined with the identity (3.11) applied with $a=\frac{\alpha}{2}, b=$ $1+\frac{\alpha}{2}$ and $c=1$ we find the compact formula

$$
\begin{aligned}
\tilde{\delta}_{n}-\tilde{\beta}_{n}= & n F\left(\frac{\alpha}{2}, 1+\frac{\alpha}{2} ; 2 ; b^{2}\right)-b^{2} \frac{\alpha}{4}\left(1+\frac{\alpha}{2}\right) F\left(1+\frac{\alpha}{2}, 2+\frac{\alpha}{2} ; 3 ; b^{2}\right) \\
& +F\left(\frac{\alpha}{2}, \frac{\alpha}{2}+1 ; 1 ; b^{2}\right) \\
= & (n+1) F\left(\frac{\alpha}{2}, 1+\frac{\alpha}{2} ; 2 ; b^{2}\right) .
\end{aligned}
$$

Putting together the preceding identities allows to write

$$
\begin{aligned}
\mathcal{L}_{3}\left(h_{1}, h_{2}(w)\right) & =i \frac{\alpha}{4} \sum_{n \geq 1}(n+1)\left(w^{n+1}-\bar{w}^{n+1}\right) \\
& \times\left[c_{n} F\left(\frac{\alpha}{2}, 1+\frac{\alpha}{2} ; 2 ; b^{2}\right)-a_{n} b^{n} \frac{\left(1+\frac{\alpha}{2}\right)_{n}}{(n+1) !} F\left(\frac{\alpha}{2}, n+1+\frac{\alpha}{2}, n+2, b^{2}\right)\right] .
\end{aligned}
$$

According to the identity (5.24) we get

$$
C_{\alpha} \mathcal{L}_{3}\left(h_{1}, h_{2}(w)\right)=\frac{i}{2} \sum_{n \geq 1}(n+1)\left(c_{n} \Lambda_{1}(b)-a_{n} \Lambda_{n+1}(b)\right)\left(w^{n+1}-\bar{w}^{n+1}\right) .
$$

Finaly, inserting the preceding identity and the expressions (5.8) and (5.16) into (5.2) one can readily verify that

$$
\begin{aligned}
D G_{2}(\Omega, 0,0)\left(h_{1}, h_{2}\right)(w)=\frac{i}{2} \sum_{n \geq 1} & (n+1)\left[a_{n} b \Lambda_{n+1}(b)+c_{n}\left(b \Omega+b^{1-\alpha} \Theta_{n+1}-b \Lambda_{1}(b)\right)\right] \\
& \times\left(w^{n+1}-\bar{w}^{n+1}\right) .
\end{aligned}
$$

This concludes the proof of the Lemma 5.1.

5.2. Asymptotic behavior. We shall collect some useful properties on the asymptotic behavior of the sequences $\left(\Theta_{n}\right)_{n}$ and $\left(\Lambda_{n}\right)_{n}$ introduced in Lemma 5.1. The study is done with respect to the parameters $\alpha$ and $n$. This is summarized in the next lemma.

Lemma 5.2. Let $\alpha \in(0,1)$ and $b \in(0,1)$. Then the following results hold true.

(1) For all $n \in \mathbb{N}^{*}, \Theta_{n} \geq 0, \Lambda_{n} \geq 0$. Moreover, $b \mapsto \Lambda_{n}(b)$ is strictly increasing, $n \mapsto \Theta_{n}$ is strictly increasing and $n \mapsto \Lambda_{n}(b)$ is strictly decreasing.

(2) Let $n \geq 2$, then

and

$$
\lim _{\alpha \rightarrow 0} \Theta_{n}=\frac{n-1}{2 n}, \quad \lim _{\alpha \rightarrow 0} \Lambda_{n}(b)=\frac{b^{n-1}}{2 n}
$$

$$
\lim _{\alpha \rightarrow 1} \Theta_{n}=\frac{2}{\pi} \sum_{k=1}^{n-1} \frac{1}{2 k+1}, \quad \lim _{\alpha \rightarrow 1} \Lambda_{n}(b)=\frac{1}{b} \int_{0}^{+\infty} J_{n}(b t) J_{n}(t) d t .
$$


(3) For $n$ sufficiently large,

$$
\begin{gathered}
\Lambda_{n}(b)=O\left(b^{n-1}\right), \quad \lim _{n \rightarrow \infty} \Lambda_{n}(b)=0, \\
\Theta_{n}=\Lambda_{1}(1)-(1-\alpha / 2) \Lambda_{1}(1) \frac{e^{\alpha \gamma+c_{\alpha}}}{n^{1-\alpha}}+O\left(\frac{1}{n^{2-\alpha}}\right), \quad \lim _{n \rightarrow \infty} \Theta_{n}=\Lambda_{1}(1) .
\end{gathered}
$$

(4) The determinant of the matrix $M_{n}^{\alpha}$ introduced in (5.5) satisfies

$$
\operatorname{det}\left(M_{n}^{\alpha}\right)=\mu+\frac{\nu}{n^{1-\alpha}}+O\left(\frac{1}{n^{2-\alpha}}\right) \text { and } \lim _{n \rightarrow+\infty} \operatorname{det}\left(M_{n}\right)=\mu,
$$

with

$$
\mu \triangleq\left(\Omega-\Lambda_{1}(1)+b^{2} \Lambda_{1}(b)\right)\left(b \Omega+b^{1-\alpha} \Lambda_{1}(1)-b \Lambda_{1}(b)\right)
$$

and

$$
\nu \triangleq(1-\alpha / 2) \Lambda_{1}(1)\left(2 b^{1-\alpha} \Lambda_{1}(1)+\left(b-b^{1-\alpha}\right) \Omega-b\left(1+b^{2-\alpha}\right) \Lambda_{1}(b)\right) e^{\alpha \gamma+c_{\alpha}},
$$

with $\gamma$ denotes Euler constant, $c_{\alpha}$ is the sum of the series

$$
c_{\alpha} \triangleq \sum_{m=1}^{\infty} \frac{\alpha^{2 m+1}}{2^{2 m-1}(2 m+1)} \zeta(2 m+1) .
$$

and $s \mapsto \zeta(s)$ is the Riemann zeta function.

Remark 5.3. The assertion (2) from the last lemma shows that the spectrum is continuous with respect to $\alpha$. In other words, we have

$$
M_{n}^{0} \triangleq \lim _{\alpha \rightarrow 0} M_{n}^{\alpha}=\left(\begin{array}{cc}
\Omega-\frac{n-1}{2 n}+\frac{b^{2}}{2} & -\frac{b^{n+1}}{2 n} \\
\frac{b^{n+1}}{2 n} & b\left(\Omega+\frac{n-1}{2 n}-\frac{1}{2}\right)
\end{array}\right)
$$

Hence, by the change of variable $\lambda=1-2 \Omega$ we can see that $M_{n}^{0}$ is exactly the same matrix obtained in [23]. However, for $\alpha=1$ the dispersion relation established in [1] involves the following matrix

$$
\widetilde{M}_{n}=\left(\begin{array}{cc}
\Omega-\frac{2}{\pi} \sum_{k=1}^{n-1} \frac{1}{2 k+1}+b^{2} \Lambda_{1}(b) & -b^{3} \Lambda_{n}(b) \\
\frac{1}{b} \Lambda_{n}(b) & \Omega+\frac{2}{b \pi} \sum_{k=1}^{n-1} \frac{1}{2 k+1}-\Lambda_{1}(b)
\end{array}\right) .
$$

This discrepancy with the matrix $M_{n}^{1}$ is due to the parametrization used in [1] for the interior curve. Indeed, in that paper the perturbation of the interior curve is dilated by $b$. Thus with our parametrization we should multiply the second column of $\widetilde{M}_{n}$ by $b$ and the matrix $\widetilde{M}_{n}$ becomes

$$
\left(\begin{array}{cc}
\Omega-\frac{2}{\pi} \sum_{k=1}^{n-1} \frac{1}{2 k+1}+b^{2} \Lambda_{1}(b) & -b^{4} \Lambda_{n}(b) \\
\frac{1}{b} \Lambda_{n}(b) & b \Omega+\frac{2}{\pi} \sum_{k=1}^{n-1} \frac{1}{2 k+1}-b \Lambda_{1}(b)
\end{array}\right) .
$$


As we can easily see, this matrix has exactly the same determinant of the matrix $M_{n}^{1}$ and therefore we find the same dispersion relations.

Let us now prove Lemma 5.2.

Proof. (1) To study the sign of $\Lambda_{n}$ we shall make use of Sonine-Schafheitlin's formula (3.18) leading to the identity

$$
\Lambda_{n}(b)=\frac{\Gamma\left(\frac{\alpha}{2}\right)}{2^{1-\alpha} \Gamma\left(1-\frac{\alpha}{2}\right)} \frac{\left(\frac{\alpha}{2}\right)_{n}}{n !} b^{n-1} F\left(\frac{\alpha}{2}, n+\frac{\alpha}{2}, n+1, b^{2}\right),
$$

which is obviously positive for all $\alpha, b \in(0,1)$.

Let us now prove that the mapping $n \mapsto \Lambda_{n}(b)$ is decreasing. For this end, we rewrite the hypergeometric series $F$ appearing in right-hand side of (5.24) according to the identity (3.3), which yields

$$
F\left(\frac{\alpha}{2}, n+\frac{\alpha}{2}, n+1, b^{2}\right)=\frac{\Gamma(n+1)}{\Gamma\left(n+\frac{\alpha}{2}\right) \Gamma\left(1-\frac{\alpha}{2}\right)} \int_{0}^{1} x^{n-1+\frac{\alpha}{2}}(1-x)^{-\frac{\alpha}{2}}\left(1-b^{2} x\right)^{-\frac{\alpha}{2}} \mathrm{~d} x .
$$

From the relation (3.5) we get

$$
\Lambda_{n}(b)=\frac{b^{n-1}}{2^{1-\alpha} \Gamma^{2}\left(1-\frac{\alpha}{2}\right)} \int_{0}^{1} x^{n-1+\frac{\alpha}{2}}(1-x)^{-\frac{\alpha}{2}}\left(1-b^{2} x\right)^{-\frac{\alpha}{2}} \mathrm{~d} x, \quad b \in(0,1) .
$$

Therefore it is easily seen that $b \in(0,1) \mapsto \Lambda_{n}(b)$ is increasing and $n \in \mathbb{N}^{\star} \mapsto \Lambda_{n}(b)$ is decreasing. This implies in turn that $n \in \mathbb{N}^{\star} \mapsto \Theta_{n}=\Lambda_{1}(1)-\Lambda_{n}(1)$ is increasing and thus it should be positive. Notice that these properties can be also proven from the series expansion (5.24).

(2) Passing to the limit in the formula defining $\Lambda_{n}(b)$ when $\alpha$ goes to one yields

$$
\lim _{\alpha \rightarrow 1} \Lambda_{n}(b)=\frac{1}{b} \int_{0}^{+\infty} J_{n}(b t) J_{n}(t) d t .
$$

As to the second limit, we have

$$
\lim _{\alpha \rightarrow 0} \Lambda_{n}(b)=\frac{1}{b} \int_{0}^{+\infty} \frac{J_{n}(b t) J_{n}(t)}{t} d t
$$

Since $b \in(0,1)$ we can use the following identity,

$$
\int_{0}^{+\infty} \frac{J_{n}(b t) J_{n}(t)}{t} d t=\frac{b^{n}}{2 n}
$$

whose proof can be found for example in [44, p. 405]. Consequently,

$$
\lim _{\alpha \rightarrow 0} \Lambda_{n}(b)=\frac{b^{n-1}}{2 n} \text {. }
$$

Now to compute the limits of $\Theta_{n}$ when $\alpha$ goes to the values 0 and 1 we shall rewrite $\Theta_{n}$ by using the identity(5.24) in the form

$$
\begin{aligned}
\Theta_{n} & \triangleq \Lambda_{1}(1)-\Lambda_{n}(1) \\
& =\frac{\left(\frac{\alpha}{2}\right) \Gamma\left(\frac{\alpha}{2}\right)}{2^{1-\alpha} \Gamma\left(1-\frac{\alpha}{2}\right)}\left[F\left(\frac{\alpha}{2}, 1+\frac{\alpha}{2}, 2,1\right)-\frac{\left(\frac{\alpha}{2}+1\right)_{n-1}}{n !} F\left(\frac{\alpha}{2}, n+\frac{\alpha}{2}, n+1,1\right)\right] .
\end{aligned}
$$


This gives in view of the formula (3.9) and (3.5),

$$
\Theta_{n}=\frac{\Gamma(1-\alpha)}{2^{1-\alpha} \Gamma^{2}\left(1-\frac{\alpha}{2}\right)}\left(\frac{\Gamma\left(n+\frac{\alpha}{2}\right)}{\Gamma\left(2-\frac{\alpha}{2}\right)}-\frac{\Gamma\left(n+\frac{\alpha}{2}\right)}{\Gamma\left(n+1-\frac{\alpha}{2}\right)}\right) .
$$

This expression coincides with the "eigenvalues" in the simply connected case, see [20]. It follows that,

$$
\begin{aligned}
\lim _{\alpha \rightarrow 0} \Theta_{n} & =\frac{1}{2}\left(\frac{\Gamma(1)}{\Gamma(2)}-\frac{\Gamma(n)}{\Gamma(n+1)}\right) \\
& =\frac{1}{2}\left(1-\frac{(n-1) !}{n !}\right) \\
& =\frac{n-1}{2 n} .
\end{aligned}
$$

We note that these values coincide with the "eigenvalues" for Euler equations in the simply connected case. To compute the second limit, we shall introduce for a fixed $n$ the function

$$
\phi_{n}(\alpha)=\frac{\Gamma(n+\alpha / 2)}{\Gamma(n+1-\alpha / 2)} .
$$

Therefore we obtain according to (3.4), (3.6) and the relation $\phi_{n}(1)=1$,

$$
\begin{aligned}
\lim _{\alpha \rightarrow 1} \Theta_{n} & =\frac{-1}{\Gamma^{2}(1 / 2)} \lim _{\alpha \rightarrow 1}\{(1-\alpha) \Gamma(1-\alpha)\} \lim _{\alpha \rightarrow 1}\left\{\frac{\phi_{1}(\alpha)-\phi_{1}(1)}{\alpha-1}-\frac{\phi_{n}(\alpha)-\phi_{n}(1)}{\alpha-1}\right\} \\
& =\frac{-1}{\pi}\left\{\phi_{1}^{\prime}(1)-\phi_{n}^{\prime}(1)\right\} .
\end{aligned}
$$

By applying the logarithm function to $\phi_{n}$ and differentiating with respect to $\alpha$ one obtains the relation

$$
2 \frac{\phi_{n}^{\prime}(\alpha)}{\phi_{n}(\alpha)}=\digamma(n+\alpha / 2)+\digamma(n+1-\alpha / 2) .
$$

Now using the fact that $\phi_{n}(1)=1$ combined with the preceding identity and (3.8), we find

$$
\begin{aligned}
\lim _{\alpha \rightarrow 1} \Theta_{n} & =\frac{-1}{\pi}\{\digamma(3 / 2)-\digamma(n+1 / 2)\} \\
& =\frac{2}{\pi} \sum_{k=1}^{n-1} \frac{1}{2 k+1},
\end{aligned}
$$

which is the desired result.

(3)-(4) The asymptotic behavior of $\Lambda_{n}$ may be easily obtained from the integral formula (5.25). The proof of (5.22) was done in details in [20]. Finally, by combining (5.22), (5.21) and the expression of $\operatorname{det}\left(M_{n}^{\alpha}\right)$ given by (5.6) one can deduce the identity (5.23).

5.3. Monotonicity of the eigenvalues. In this section we shall discuss some important properties concerning the monotonicity of the eigenvalues associated to the matrix $M_{n}^{\alpha}$ already seen in Lemma 5.1. This will be crucial in the study of the kernel of the linearized operator $D G(\Omega, 0,0)$. Recall that

$$
M_{n}^{\alpha}=\left(\begin{array}{cc}
\Omega-\Theta_{n}+b^{2} \Lambda_{1}(b) & -b^{2} \Lambda_{n}(b) \\
b \Lambda_{n}(b) & b \Omega+b^{1-\alpha} \Theta_{n}-b \Lambda_{1}(b)
\end{array}\right) .
$$


The determinant of this matrix given by (5.6) is a second order polynomial on the variable $\Omega$ and therefore it has two roots depending on all the parameters $n, b$ and $\alpha$. For our deal it is important to formulate sufficient conditions to avoid the eigenvalues crossing in order to guarantee a one-dimensional kernel which is an essential assumption in CrandallRabionwitz's Theorem. In what follows we shall use the variable $\lambda \triangleq 1-2 \Omega$ instead of $\Omega$ in the spirit of the work of [23]. Thus easy computations show that the determinant (5.6) takes the form,

$$
\operatorname{det}\left(M_{n}^{\alpha}\right)=b\left(\lambda^{2}-2 C_{n} \lambda+D_{n}\right)
$$

with

$$
C_{n} \triangleq 1+\left(b^{-\alpha}-1\right) \Theta_{n}-\left(1-b^{2}\right) \Lambda_{1}(b),
$$

and

$$
\begin{gathered}
D_{n} \triangleq-4 b^{-\alpha} \Theta_{n}^{2}+2\left[b^{-\alpha}-1+2\left(1+b^{2-\alpha}\right) \Lambda_{1}(b)\right] \Theta_{n}-4 b^{2}\left(\Lambda_{1}^{2}(b)-\Lambda_{n}^{2}(b)\right) \\
-2\left(1-b^{2}\right) \Lambda_{1}(b)+1 .
\end{gathered}
$$

Note that the quantities $\Lambda_{n}(b)$ and $\Theta_{n}$ have been introduced in Lemma 5.1. It is easy to check through straightforward computations that the reduced discriminant of the second order polynomial appearing in (5.27) is given by

$$
\Delta_{n}=\left(\left(b^{-\alpha}+1\right) \Theta_{n}-\left(1+b^{2}\right) \Lambda_{1}(b)\right)^{2}-4 b^{2} \Lambda_{n}^{2}(b) .
$$

Our result reads as follows.

Proposition 5.4. There exists $N \geq 2$ such that the following holds true.

(1) For all $n>N$ we get $\Delta_{n}>0$ and the equation (5.27) admits two different real solutions given by

$$
\lambda_{n}^{ \pm} \triangleq C_{n} \pm \sqrt{\Delta_{n}}
$$

(2) The sequences $\left(\Delta_{n}\right)_{n \geq N}$ and $\left(\lambda_{n}^{+}\right)_{n \geq N}$ are strictly increasing and $\left(\lambda_{n}^{-}\right)_{n \geq N}$ is strictly decreasing.

(3) For all $m>n>N$ we have

$$
\lambda_{m}^{-}<\lambda_{n}^{-}<\lambda_{n}^{+}<\lambda_{m}^{+}
$$

Remark 5.5. (1) The number $N$ in (2) of the previous proposition is the smallest integer satisfying,

$$
\Theta_{N} \geq \frac{1+b^{2}}{b^{-\alpha}+1} \Lambda_{1}(b)+\frac{2 b}{b^{-\alpha}+1} \Lambda_{N}(b) .
$$

(2) In the known cases like the simply connected case with $\alpha \in[0,2[$ or the doubly connected case with $\alpha=0$ the analysis is more easier because the dispersion relation is a kind of fractional polynomial but in the present case it is highly nonlinear with respect to the frequencies and the parameters $\alpha$ and $b$. Therefore the program is achieved with only a sufficient condition on the existence of the eigenvalue and which is given by (5.30). This condition coincides with that given in [23] for $\alpha=0$. 
Proof. (1) We intend to discuss the conditions leading to the positivity of the discriminant defined in (5.29) which ensures in turn that the polynomial (5.27) has two real solutions. We can see that $\left(\Delta_{n}\right)_{n \geq 2}$ can be extended to a smooth function defined on $[1,+\infty$ [ as follows

$$
\Delta_{x}=\left(\left(b^{-\alpha}+1\right) \Theta_{x}-\left(1+b^{2}\right) \Lambda_{1}(b)\right)^{2}-4 b^{2} \Lambda_{x}^{2}(b) .
$$

It is strictly positive if and only if

$$
E_{b}(x) \triangleq\left(b^{-\alpha}+1\right) \Theta_{x}-\left(1+b^{2}\right) \Lambda_{1}(b)-2 b \Lambda_{x}(b)>0,
$$

or

$$
F_{b}(x) \triangleq\left(b^{-\alpha}+1\right) \Theta_{x}-\left(1+b^{2}\right) \Lambda_{1}(b)+2 b \Lambda_{x}(b)<0 .
$$

Remark first that for $x$ and $b$ verifying the inequality (5.31) we have $F_{b}(x)>0$. On other hand, from Lemma 5.2 one has : for all $b \in(0,1)$ the mapping $x \mapsto E_{b}(x)$ is strictly increasing, continuous and satisfying

$$
\begin{gathered}
\lim _{x \rightarrow+\infty} E_{b}(x)=\left(b^{-\alpha}+1\right) \Lambda_{1}(1)-\left(1+b^{2}\right) \Lambda_{1}(b) \\
\geq\left(b^{-\alpha}-b^{2}\right) \Lambda_{1}(1)>0 \\
E_{b}(1)=-(1+b)^{2} \Lambda_{1}(b)<0 .
\end{gathered}
$$

Consequently the set

$$
\mathcal{I}^{\alpha}(b) \triangleq\left\{x>1 ; E_{b}(x)>0\right\} .
$$

is connected and takes the form $] \beta,+\infty[$, with

$$
E_{b}(\beta)=0 \text {. }
$$

Hence the integer $N$ is chosen as

$$
N=[\beta]
$$

(2) To prove that $x \mapsto \Delta_{x}$ is decreasing we shall compute its derivative with respect to $x$. Pain computations give,

$$
\partial_{x} \Delta_{x}=2\left(b^{-\alpha}+1\right)\left[\left(b^{-\alpha}+1\right) \Theta_{x}-\left(1+b^{2}\right) \Lambda_{1}(b)\right] \partial_{x} \Theta_{x}-8 b^{2} \Lambda_{x}(b) \partial_{x} \Lambda_{x}(b) .
$$

Since $x \mapsto \Lambda_{x}(b)$ is decreasing, $x \mapsto \Theta_{x}$ is increasing and $\Lambda_{x}(b) \geq 0$ (see (1) from Lemma 5.2) then we deduce that

$$
\begin{aligned}
\partial_{x} \Delta_{x} & >2\left(b^{-\alpha}+1\right)\left[\left(b^{-\alpha}+1\right) \Theta_{x}-\left(1+b^{2}\right) \Lambda_{1}(b)\right] \partial_{x} \Theta_{x} \\
& >2\left(b^{-\alpha}+1\right) E_{x}(b) \partial_{x} \Theta_{x} .
\end{aligned}
$$

Hence, for all $x \in \mathcal{I}^{\alpha}(b)$ we have

$$
\partial_{x} \Delta_{x}>0
$$

This shows that $x \mapsto \Delta_{x}$ is strictly increasing.

Now, recall that

$$
\lambda_{x}^{+} \triangleq 1+\left(b^{-\alpha}-1\right) \Theta_{x}-\left(1-b^{2}\right) \Lambda_{1}(b)+\sqrt{\Delta_{x}} .
$$

Using the fact that $x \mapsto \Theta_{x}$ is increasing (according to Lemma 5.2) combined with the increasing property of $x \mapsto \Delta_{x}$ we get the desired result. 
So it remains to establish that the mapping $x \mapsto \lambda_{x}^{-}$is strictly decreasing. For this aim we calculate its derivative with respect to $x$,

$$
\begin{aligned}
\partial_{x} \lambda_{x}^{-}= & \left(b^{-\alpha}-1\right) \partial_{x} \Theta_{x}-\frac{\partial_{x} \Delta_{x}}{2 \sqrt{\Delta_{x}}} \\
= & \left(b^{-\alpha}-1\right) \partial_{x} \Theta_{x}-\left(b^{-\alpha}+1\right)\left[\frac{\left(b^{-\alpha}+1\right) \Theta_{x}-\left(1+b^{2}\right) \Lambda_{1}(b)}{\sqrt{\Delta_{x}}}\right] \partial_{x} \Theta_{x}+4 b^{2} \partial_{x} \Lambda_{x}(b) \frac{\Lambda_{x}(b)}{\sqrt{\Delta_{x}}} \\
& <b^{-\alpha}\left[1-\frac{\left(b^{-\alpha}+1\right) \Theta_{x}-\left(1+b^{2}\right) \Lambda_{1}(b)}{\sqrt{\Delta_{x}}}\right] \partial_{x} \Theta_{x} \\
& -\left[1+\frac{\left(b^{-\alpha}+1\right) \Theta_{x}-\left(1+b^{2}\right) \Lambda_{1}(b)}{\sqrt{\Delta_{x}}}\right] \partial_{x} \Theta_{x} .
\end{aligned}
$$

where we have used in the last inequality the decreasing property of the mapping $x \mapsto \Lambda_{x}(b)$ and the fact that $\Lambda_{x}(b) \geq 0$. Now from the inequality

$$
\left(b^{-\alpha}+1\right) \Theta_{x}-\left(1+b^{2}\right) \Lambda_{1}(b)>E_{x}(b)>0,
$$

and the expression of $\Delta_{x}$ we deduce that

$$
\partial_{x} \lambda_{x}^{-}<0
$$

This gives the desired result.

(3) This follows easily from (2) and the obvious fact

$$
\lambda_{n}^{-} \leq \lambda_{n}^{+} .
$$

\section{Bifurcation at simple eigenvalues}

In this section we shall prove Theorem 1.1 which is deeply related to the spectral study developed in the preceding section combined with Crandall-Rabinowitz's Theorem. To construct the function spaces where the bifurcation occurs we shall take into account the restriction to the high frequencies stated in Proposition 5.4 and include the $m$-fold symmetry of the V-states. To proceed, fix $b \in(0,1)$ and $m>N$, where $N$ is defined in Proposition 5.4 and Remark 5.5. Set,

$$
X_{m}=C_{m}^{2-\alpha}(\mathbb{T}) \times C_{m}^{2-\alpha}(\mathbb{T}),
$$

where $C_{m}^{2-\alpha}(\mathbb{T})$ is the space of the $2 \pi$-periodic functions $f \in C^{2-\alpha}(\mathbb{T})$ whose Fourier series is given by

$$
f(w)=\sum_{n=1}^{\infty} a_{n} \bar{w}^{n m-1}, \quad w \in \mathbb{T}, \quad a_{n} \in \mathbb{R} .
$$

This space is equipped with its usual norm. We define the ball of radius $r \in(0,1)$ by

$$
B_{r}^{m}=\left\{f \in X_{m},\|f\|_{C^{2-\alpha}(\mathbb{T})} \leq r\right\}
$$

and we introduce the neighborhood of zero,

$$
V_{m, r} \triangleq B_{r}^{m} \times B_{r}^{m}
$$


The set $V_{m, r}$ is endowed with the induced topology of the product spaces.

Take $\left(f_{1}, f_{2}\right) \in V_{m, r}$ then the expansions of the associated conformal mappings $\phi_{1}, \phi_{2}$ outside the unit disc $\{z \in \mathbb{C} ;|z| \geq 1\}$ are given successively by

$$
\phi_{1}(z)=z+f_{1}(z)=z\left(1+\sum_{n=1}^{\infty} \frac{a_{n}}{z^{n m}}\right)
$$

and

$$
\phi_{2}(z)=b z+f_{2}(z)=z\left(b+\sum_{n=1}^{\infty} \frac{b_{n}}{z^{n m}}\right) .
$$

This structure provides the $m$-fold symmetry of the associated boundaries $\phi_{1}(\mathbb{T})$ and $\phi_{2}(\mathbb{T})$, via the relation

$$
\phi_{j}\left(e^{2 i \pi / m} z\right)=e^{2 i \pi / m} \phi_{j}(z), \quad j=1,2 \quad \text { and } \quad|z| \geq 1 .
$$

For functions $f_{1}$ and $f_{2}$ with small size the boundaries can be seen as a small perturbation of the boundaries of the annulus $\{z \in \mathbb{C} ; b \leq|z| \leq 1\}$. Set

$$
H_{m}=\left\{g \in C^{1-\alpha}(\mathbb{T}), g(w)=i \sum_{n \geq 1} A_{n}\left(w^{m n}-\bar{w}^{m n}\right), A_{n} \in \mathbb{R}, n \in \mathbb{N}^{*}\right\}
$$

and define the product space $Y_{m}$ by

$$
Y_{m}=H_{m} \times H_{m}
$$

From Proposition 5.4 recall the definition of the eigenvalues $\lambda_{m}^{ \pm}$and the associated angular velocities are

$$
\begin{aligned}
\Omega_{m}^{ \pm} & =\frac{1}{2}-\frac{1}{2} \lambda_{m}^{ \pm} \\
& =\frac{1}{2} \widehat{C}_{m} \mp \frac{1}{2} \sqrt{\Delta_{m}}
\end{aligned}
$$

with

$$
\Delta_{m} \triangleq\left(\left(b^{-\alpha}+1\right) \Theta_{m}-\left(1+b^{2}\right) \Lambda_{1}(b)\right)^{2}-4 b^{2} \Lambda_{m}^{2}(b)
$$

and

$$
\widehat{C}_{m} \triangleq\left(1-b^{-\alpha}\right) \Theta_{m}+\left(1-b^{2}\right) \Lambda_{1}(b)
$$

Note that $\Theta_{m}$ and $\Lambda_{m}(b)$ were introduced in Lemma 5.1. The V-states equations are described in (2.2) and (2.3) which we restate here: for $j \in\{1,2\}$,

$$
F_{j}\left(\Omega, \phi_{1}, \phi_{2}\right)(w) \triangleq G_{j}\left(\Omega, f_{1}, f_{2}\right)(w)=0, \quad \forall w \in \mathbb{T} ; \quad \text { and } \quad G \triangleq\left(G_{1}, G_{2}\right) .
$$

with

$$
\begin{aligned}
F_{j}\left(\Omega, \phi_{1}, \phi_{2}\right)(w) & \triangleq \Omega \operatorname{Im}\left\{\phi_{j}(w) \bar{w} \overline{\phi_{j}^{\prime}(w)}\right\} \\
& +C_{\alpha} \operatorname{Im}\left\{\left(f_{\mathbb{T}} \frac{\phi_{2}^{\prime}(\tau) d \tau}{\left|\phi_{j}(w)-\phi_{2}(\tau)\right|^{\alpha}}-f_{\mathbb{T}} \frac{\phi_{1}^{\prime}(\tau) d \tau}{\left|\phi_{j}(w)-\phi_{1}(\tau)\right|^{\alpha}}\right) \bar{w} \overline{\phi_{j}^{\prime}(w)}\right\} .
\end{aligned}
$$

Now, to apply Crandall-Rabinowitz's Theorem it suffices to show the following result. 
Proposition 6.1. Let $N$ be as in the part (1) of Proposition 5.4 and $m \geq N$, and take $\Omega \in\left\{\Omega_{m}^{ \pm}\right\}$. Then, the following assertions hold true.

(1) There exists $r>0$ such that $G: \mathbb{R} \times V_{m, r} \rightarrow Y_{m}$ is well-defined and of class $C^{1}$.

(2) The kernel of $D G(\Omega, 0,0)$ is one-dimensional and generated by

$$
v_{0, m}: w \in \mathbb{T} \mapsto\left(\begin{array}{c}
\Omega+b^{-\alpha} \Theta_{m}-\Lambda_{1}(b) \\
-\Lambda_{m}(b)
\end{array}\right) \bar{w}^{m-1} .
$$

(3) The range of $D G(\Omega, 0,0)$ is closed and is of co-dimension one in $Y_{m}$.

(4) Transversality assumption: If $\Omega$ is a simple eigenvalue $\left(\Delta_{m}>0\right)$ then

$$
\partial_{\Omega} D G\left(\Omega_{m}^{ \pm}, 0,0\right) v_{0, m} \notin R\left(D G\left(\Omega_{m}^{ \pm}, 0,0\right)\right) .
$$

Proof. (1) Compared to Proposition 4.1 we need just to check that $G=\left(G_{1}, G_{2}\right)$ preserves the $m$-fold symmetry and maps $X_{m}$ into $Y_{m}$. For this end, it is sufficient to check that for given $\left(f_{1}, f_{2}\right) \in X_{m}$, the coefficients of the Fourier series of $F_{j}\left(\Omega, \phi_{1}, \phi_{2}\right)$ vanish at frequencies which are not integer multiple of $m$. This amounts to proving that,

$$
F_{j}\left(\Omega, \phi_{1}, \phi_{2}\right)\left(e^{i \frac{2 \pi}{m}} w\right)=F_{j}\left(\Omega, \phi_{1}, \phi_{2}\right)(w), \quad w \in \mathbb{T}, \quad j=1,2 .
$$

This property is obvious for the first term $\operatorname{Im}\left\{\bar{w} \overline{\phi_{j}^{\prime}(w)} \phi_{j}(w)\right\}$. For the two last terms in the expression of $F_{j}$ it is enough to check the identity,

$$
\forall w \in \mathbb{T}, \quad \Phi_{j}\left(e^{i \frac{2 \pi}{m}} w\right)=e^{i \frac{2 \pi}{m}} \Phi_{j}(w),
$$

with

$$
\Phi_{j}(w) \triangleq f_{\mathbb{T}} \frac{\phi_{2}^{\prime}(\tau)}{\left|\phi_{j}(w)-\phi_{2}(\tau)\right|^{\alpha}} d \tau
$$

This follows easily by making the change of variables $\tau=e^{i 2 \pi / m} \zeta$ and from (6.1). Indeed,

$$
\begin{aligned}
\Phi_{j}\left(e^{i \frac{2 \pi}{m}} w\right) & =e^{i 2 \pi / m} f_{\mathbb{T}} \frac{\phi_{2}^{\prime}\left(e^{i 2 \pi / m} \zeta\right)}{\left|\phi_{j}\left(e^{i \frac{2 \pi}{m}} w\right)-\phi_{2}\left(e^{i 2 \pi / m} \zeta\right)\right|^{\alpha}} d \zeta \\
& =e^{i \frac{2 \pi}{m}} f_{\mathbb{T}} \frac{\phi_{2}^{\prime}(\zeta)}{\left|\phi_{j}(w)-\phi_{2}(\zeta)\right|^{\alpha}} d \zeta \\
& =e^{i \frac{2 \pi}{m}} \Phi_{j}(w) .
\end{aligned}
$$

This concludes the proof of the following statement,

$$
\left(f_{1}, f_{2}\right) \in V_{m, r} \Longrightarrow G\left(\Omega, f_{1}, f_{2}\right) \in Y_{m} .
$$

(2) We shall describe the kernel of the linear operator $D F\left(\Omega_{m}^{ \pm}, 0,0\right)$ and show that it is one-dimensional. Let $h_{1}, h_{2}$ be two functions in $C_{m}^{2-\alpha}(\mathbb{T})$ such that

$$
h_{1}(w)=\sum_{n=1}^{\infty} a_{n} \bar{w}^{n m-1} \quad \text { and } \quad h_{2}(w)=\sum_{n=1}^{\infty} c_{n} \bar{w}^{n m-1}, \quad w \in \mathbb{T},
$$

Recall from Lemma 5.1 the following expression,

$$
D G(\Omega, 0,0)\left(h_{1}, h_{2}\right)(w)=\frac{i}{2} \sum_{n \geq 1} n m M_{n m}^{\alpha}\left(\begin{array}{c}
a_{n} \\
c_{n}
\end{array}\right)\left(w^{n m}-\bar{w}^{n m}\right) .
$$


where the matrix $M_{n}$ is given for $n \geq 2$ by

$$
M_{n}^{\alpha} \triangleq\left(\begin{array}{cc}
\Omega-\Theta_{n}+b^{2} \Lambda_{1}(b) & -b^{2} \Lambda_{n}(b) \\
b \Lambda_{n}(b) & b \Omega+b^{1-\alpha} \Theta_{n}-b \Lambda_{1}(b)
\end{array}\right) .
$$

Now, if $\Omega \in\left\{\Omega_{m}^{ \pm}\right\}$then

$$
\operatorname{det}\left(M_{m}^{\alpha}\right)=0 .
$$

Thus, the kernel of $D G(\Omega, 0,0)$ is non trivial and it is one-dimensional if and only if

$$
\operatorname{det}\left(M_{n m}^{\alpha}\right) \neq 0, \quad \forall n \geq 2 .
$$

This condition is ensured by the part (1) of the Proposition 5.4. Then, $\left(h_{1}, h_{2}\right)$ is in the kernel of $D G(\Omega, 0,0)$ if and only the Fourier coefficients in the identity (6.3) vanish, namely,

$$
a_{n}=c_{n}=0 \text { for all } n \geq 2 \text { and }\left(a_{1}, c_{1}\right) \in \operatorname{Ker} M_{m}^{\alpha} .
$$

Hence, a generator of $\operatorname{Ker}(D G(\Omega, 0,0))$ can be chosen as the pair of functions

$$
w \in \mathbb{T} \mapsto\left(\begin{array}{c}
\Omega+b^{-\alpha} \Theta_{m}-\Lambda_{1}(b) \\
-\Lambda_{m}(b)
\end{array}\right) \bar{w}^{m-1}, \quad w \in \mathbb{T} .
$$

(3) We are going to show that for any $m \geq N$ the range $R(D G(\Omega, 0,0))$ coincides with the space of the functions $\left(g_{1}, g_{2}\right) \in C^{1-\alpha}(\mathbb{T}) \times C^{1-\alpha}(\mathbb{T})$ such that

$$
g_{1}(w)=\sum_{n \geq 1} i A_{n}\left(w^{m n}-\bar{w}^{m n}\right), \quad g_{1}(w)=\sum_{n \geq 1} i C_{n}\left(w^{m n}-\bar{w}^{m n}\right)
$$

where $A_{n}, C_{n} \in \mathbb{R}$ for all $n \in \mathbb{N}^{*}$ and there exists $\left(a_{1}, c_{1}\right) \in \mathbb{R}^{2}$ such that

$$
M_{m}\left(\begin{array}{c}
a_{1} \\
c_{1}
\end{array}\right)=\left(\begin{array}{l}
A_{1} \\
C_{1}
\end{array}\right) .
$$

For the sake of simple notation we remove in this part the parameter $\alpha$ from $M_{n}^{\alpha}$. The range of operator $D G(\Omega, 0,0)$ is obviously included in the space defined above which is closed and of co-dimension 1 in $Y_{m}$. Therefore it remains to check just the converse. Let $g_{1}$ and $g_{2}$ be two functions in $C^{1-\alpha}(\mathbb{T})$ with Fourier series expansions as in (6.7) and (6.8). We shall prove that the equation

$$
\frac{1}{m} D G(\Omega, 0,0)\left(h_{1}, h_{2}\right)=\left(g_{1}, g_{2}\right)
$$

admits a solution $\left(h_{1}, h_{2}\right)$ in the space $X_{m}$, where the Fourier series expansions of these functions are given in (6.2). Then according to (6.3), the preceding equation is equivalent to

$$
n M_{m n}\left(\begin{array}{c}
a_{n} \\
c_{n}
\end{array}\right)=\left(\begin{array}{c}
A_{n} \\
C_{n}
\end{array}\right), \quad \forall n \in \mathbb{N}^{\star} .
$$

For $n=1$, the existence follows from the condition (6.8) and therefore we shall only focus on $n \geq 2$. Owing to (6.5) the sequences $\left(a_{n}\right)_{n \geq 2}$ and $\left(c_{n}\right)_{n \geq 2}$ are uniquely determined by the formulae

$$
\left(\begin{array}{c}
a_{n} \\
c_{n}
\end{array}\right)=\frac{1}{n} M_{m n}^{-1}\left(\begin{array}{c}
A_{n} \\
C_{n}
\end{array}\right), \quad n \geq 2 .
$$


By computing the matrix $M_{m n}^{-1}$ we deduce that for all $n \geq 2$,

$$
a_{n}=\frac{b\left(\Omega+b^{-\alpha} \Theta_{n m}-\Lambda_{1}(b)\right)}{n \operatorname{det}\left(M_{n m}\right)} A_{n}+\frac{b^{2} \Lambda_{n m}(b)}{n \operatorname{det}\left(M_{n m}\right)} C_{n}
$$

and

$$
c_{n}=-\frac{b \Lambda_{n m}(b)}{n \operatorname{det}\left(M_{n m}\right)} A_{n}+\frac{\left(\Omega-b \Theta_{n m}+b^{2} \Lambda_{1}(b)\right)}{n \operatorname{det}\left(M_{n m}\right)} C_{n} .
$$

Therefore the proof of $\left(h_{1}, h_{2}\right) \in X_{m}$ amounts to showing that

$$
w \mapsto\left(\begin{array}{c}
h_{1}(w)-a_{1} \bar{w}^{m-1} \\
h_{2}(w)-c_{1} \bar{w}^{m-1}
\end{array}\right) \in C^{2-\alpha}(\mathbb{T}) \times C^{2-\alpha}(\mathbb{T}) .
$$

We shall develop the computations only for the first component and the second one can be done in a similar way. We set $\widetilde{h}_{1}(w)=h_{1}(w)-a_{1} \bar{w}^{m-1}$ and

$$
H(w) \triangleq \sum_{n \geq 2} \frac{A_{n}}{n \operatorname{det}\left(M_{n m}\right)} w^{n}, \quad H_{1}(w) \triangleq \sum_{n \geq 2} \frac{C_{n}}{n} w^{n} .
$$

Then in view of $(5.22)$ the function $\widetilde{h}_{1}(w)$ can be rewritten as follows

$$
\begin{aligned}
\widetilde{h}_{1}(w) & =C_{1} w H\left(\bar{w}^{m}\right)+C_{2} w\left(H * K_{1}\right)\left(\bar{w}^{m}\right) \\
& +w\left(H * K_{2}\right)\left(\bar{w}^{m}\right)+b^{2} w\left(H_{1} * K_{3}\right)\left(\bar{w}^{m}\right),
\end{aligned}
$$

with $C_{1}$ and $C_{2}$ two constants. The kernels are defined by

$$
K_{1}(w) \triangleq \sum_{n \geq 2} \frac{w^{n}}{n^{1-\alpha}}, \quad K_{2}(w) \triangleq \sum_{n \geq 2} O\left(\frac{1}{n^{2-\alpha}}\right) w^{n},
$$

and

$$
K_{3}(w) \triangleq \sum_{n \geq 2} \frac{\Lambda_{n m}(b)}{\operatorname{det}\left(M_{n m}\right)} w^{n} .
$$

The convolution is understood in the usual one: for two continuous functions $f, g ; \mathbb{T} \rightarrow \mathbb{C}$ we define

$$
\forall w \in \mathbb{T}, \quad f * g(w)=f_{\mathbb{T}} f(\tau) g(\tau \bar{w}) \frac{d \tau}{\tau} .
$$

Assume for a while that $w \mapsto H(w)$ belongs to $C^{2-\alpha}(\mathbb{T})$. Then by virtue of the classical convolution law $L^{1}(\mathbb{T}) * C^{2-\alpha}(\mathbb{T}) \rightarrow C^{2-\alpha}(\mathbb{T})$, it suffices to show that the kernels $K_{1}, K_{2}$ and $K_{3}$ belong to $L^{1}(\mathbb{T})$. The second and the third kernels are easy to analyze because the series converge absolutely,

$$
\left\|K_{2}\right\|_{L^{\infty}(\mathbb{T})} \lesssim \sum_{n \geq 1} \frac{1}{n^{2-\alpha}} \leq C .
$$

Similarly, owing to (5.21) one has

$$
\left\|K_{3}\right\|_{L^{\infty}(\mathbb{T})} \lesssim \sum_{n \geq 0} b^{n} \leq C
$$

and therefore $K_{2}, K_{3} \in L^{1}(\mathbb{T})$. Note that to bound the series we have used the fact that the sequence $\left(\operatorname{det} M_{n m}\right)_{n \geq 2}$ does not vanish and converges to a strictly positive number $D_{\infty}$ 
defined in (??). It remains to show that $K_{1} \in L^{1}(\mathbb{T})$. For this end we shall use the following estimate: for any $\beta \in(\alpha, 1)$

$$
\left|K_{1}\left(e^{i \theta}\right)\right| \lesssim \frac{1}{\sin ^{\beta}\left(\frac{\theta}{2}\right)}, \quad \forall \theta \in(0,2 \pi) .
$$

which is true for all $\alpha \in[0,1$ [ and for a proof we can see [20].

Now to complete the reasoning it remains to prove the preceding claim asserting that the function $H$ belongs to the space $C^{2-\alpha}(\mathbb{T})$. To prove this we write in view of $(5.23)$,

$$
\begin{aligned}
\operatorname{det}\left(M_{n m}\right) & =\mu+\frac{\nu}{n^{1-\alpha}}+O\left(\frac{1}{n^{2-\alpha}}\right) \\
& =\mu-\rho_{n},
\end{aligned}
$$

where $\mu$ and $\nu$ are two constants (depending on $m$ ) and

$$
\rho_{n} \triangleq-\frac{\nu}{n^{1-\alpha}}+O\left(\frac{1}{n^{2-\alpha}}\right)
$$

This allows to get

$$
H(w)=\sum_{n \geq 2} \frac{A_{n}}{n\left(\mu-\rho_{n}\right)} w^{n} .
$$

Then one may use the general decomposition: for $k \in \mathbb{N}$,

$$
\frac{1}{\mu-\rho_{n}}=\frac{\mu^{-k-1} \rho_{n}^{k+1}}{\mu-\rho_{n}}+\sum_{j=0}^{k} \mu^{-j-1} \rho_{n}^{j}
$$

which yields,

$$
\begin{aligned}
H(w) & =\mu^{-k-1} \sum_{n \geq 2} \frac{A_{n} \rho_{n}^{k+1}}{n\left(\mu-\rho_{n}\right)} w^{n}+\sum_{j=0}^{k} \mu^{-j-1} \sum_{n \geq 2} \frac{A_{n} \rho_{n}^{j}}{n} w^{n} \\
& \triangleq \mu^{-k-1} H_{k+1}(w)+\sum_{j=0}^{k} \mu^{-j-1} L_{j}(w) .
\end{aligned}
$$

Since the sequence $\left(A_{n}\right)_{n \geq 2}$ is bounded then by (6.11) we obtain

$$
\left|\frac{A_{n} \rho_{n}^{k+1}}{n\left(\mu-\rho_{n}\right)}\right| \lesssim \frac{\left|\rho_{n}\right|^{k+1}}{n} \lesssim \frac{1}{n^{1+(1-\alpha)(k+1)}} .
$$

Thus for $k$ large enough we get $H_{k+1} \in C^{2-\alpha}(\mathbb{T})$. Concerning the estimate of $L_{j}$ we shall restrict the analysis to $j=0$ and $j=1$ and the higher terms can be treated in a similar way. We write

$$
L_{0}(w)=\sum_{n \geq 2} \frac{A_{n}}{n} w^{n} .
$$


Using the Cauchy-Schwarz inequality we get

$$
\begin{aligned}
\left\|L_{0}\right\|_{L^{\infty}} & \leq \sum_{n \geq 2} \frac{\left|A_{n}\right|}{n} \\
& \leq\left(\sum_{n \geq 1} \frac{1}{n^{2}}\right)^{1 / 2}\left(\sum_{n \geq 2}\left|A_{n}\right|^{2}\right)^{1 / 2} \\
& \lesssim\left\|g_{1}\right\|_{L^{2}} .
\end{aligned}
$$

By the embedding $C^{1-\alpha}(\mathbb{T}) \hookrightarrow L^{\infty}(\mathbb{T}) \hookrightarrow L^{2}(\mathbb{T})$ we conclude that

$$
\left\|L_{0}\right\|_{L^{\infty}} \lesssim\left\|g_{1}\right\|_{1-\alpha}
$$

It remains to prove that $L_{0}^{\prime} \in C^{1-\alpha}(\mathbb{T})$. For this end, one needs first to check that we can differentiate the series term by term. Fix $N \geq 1$ and define

$$
L_{0}^{N}(w) \triangleq \sum_{n=2}^{N} \frac{A_{n}}{n} w^{n} .
$$

From Cauchy-Schwarz inequality we find

$$
\begin{aligned}
\left\|L_{0}^{N}-L_{0}\right\|_{L^{\infty}(\mathbb{T})} & \lesssim\left(\sum_{n \geq N+1} \frac{1}{n^{2}}\right)^{1 / 2}\left\|g_{1}\right\|_{1-\alpha} \\
& \lesssim \frac{\left\|g_{1}\right\|_{1-\alpha}}{N^{1 / 2}} .
\end{aligned}
$$

Hence,

$$
\lim _{N \rightarrow+\infty}\left\|L_{0}^{N}-L_{0}\right\|_{L^{\infty}(\mathbb{T})}=0 .
$$

Differentiating $L_{0}^{N}$ term by term one gets

$$
\begin{aligned}
\left(L_{0}^{N}\right)^{\prime}(w) & =\bar{w} \sum_{n=2}^{N} A_{n} w^{n} \\
& \triangleq \bar{w} g_{1}^{N}(w) .
\end{aligned}
$$

Put

$$
g_{1}^{+}(w)=\sum_{n \geq 2} A_{n} w^{n}
$$

then using the continuity of Szegö protection:

$$
\Pi: \sum_{n \in \mathbb{Z}} a_{n} w^{n} \mapsto \sum_{n \in \mathbb{N}} a_{n} w^{n}
$$

on Hölder spaces $C^{1-\alpha}(\mathbb{T})$ for $\alpha \in(0,1)$ we may conclude that $g_{1}^{+}$belongs to $C^{1-\alpha}(\mathbb{T})$, (for more details see for example [20]). By virtue of a classical result on Fourier series one gets

$$
\lim _{N \rightarrow+\infty}\left\|g_{1}^{N}-g_{1}^{+}\right\|_{L^{\infty}(\mathbb{T})}=0
$$

and consequently

$$
\lim _{N \rightarrow+\infty}\left\|\left(L_{0}^{N}\right)^{\prime}-\bar{w} g_{1}^{+}\right\|_{L^{\infty}(\mathbb{T})}=0 .
$$


Putting together (6.12) and (6.13) we deduce that $L_{0}$ is differentiable and

$$
L_{0}^{\prime}(w)=\bar{w} g_{1}^{+}(w), \quad w \in \mathbb{T} .
$$

This concludes that $L_{0} \in C^{2-\alpha}$. Now, as before, we can easily get $L_{1} \in L^{\infty}(\mathbb{T})$ and we shall check that $L_{1}^{\prime} \in C^{1-\alpha}(\mathbb{T})$. Arguing in a similar way to $L_{0}$ we can differentiate term by term the series defining $L_{j}$ leading to

$$
L_{1}^{\prime}(w)=\bar{w} \sum_{n \geq 2} A_{n} \rho_{n} w^{n} .
$$

Note that with the same kernels $K_{1}$ and $K_{2}$ as before one can write

$$
w L^{\prime}{ }_{1}(w)=-\nu\left(K_{1} * g_{1}^{+}\right)(w)+\left(K_{2} * g_{1}^{+}\right)(w) .
$$

Using the fact that $g_{1}^{+}$belongs to $C^{1-\alpha}(\mathbb{T})$ and $K_{1}, K_{2} \in L^{1}(\mathbb{T})$ we obtain the desired result.

(4) The transversality condition. Let $\Omega \in\left\{\Omega_{m}^{ \pm}\right\}$be a simple eigenvalue associated to the frequencies $m$ and $v_{0, m}$ be the generator of the kernel $D G(\Omega, 0,0)$ defined in the part (2) of Proposition 6.1. We shall prove that

$$
\partial_{\Omega} D G(\Omega, 0,0) v_{0, m} \notin R(D G(\Omega, 0,0)),
$$

with

$$
v_{0, m}(w)=\left(\begin{array}{c}
\Omega+b^{-\alpha} \Theta_{m}-\Lambda_{1}(b) \\
-\Lambda_{m}(b)
\end{array}\right) \bar{w}^{m-1}, \quad w \in \mathbb{T} .
$$

Differentiating (5.1) and (5.2) with respect to $\Omega$ we get

$$
\partial_{\Omega} D G_{1}(\Omega, 0,0)\left(h_{1}, h_{2}\right)(w)=\operatorname{Im}\left\{\overline{h_{1}^{\prime}(w)}+\bar{w} h_{1}(w)\right\}
$$

and

Hence,

$$
\partial_{\Omega} D G_{2}(\Omega, 0,0)\left(h_{1}, h_{2}\right)(w)=b \operatorname{Im}\left\{\overline{h_{2}^{\prime}(w)}+\bar{w} h_{2}(w)\right\} .
$$

$$
\begin{aligned}
\partial_{\Omega} D G(\Omega, 0,0)\left(v_{0, m}\right)(w) & =\frac{m}{2} i\left(\begin{array}{c}
\Omega+b^{-\alpha} \Theta_{m}-\Lambda_{1}(b) \\
-b \Lambda_{m}(b)
\end{array}\right)\left(w^{m}-\bar{w}^{m}\right) \\
& \triangleq \frac{m}{2} i e_{m}\left(w^{m}-\bar{w}^{m}\right) .
\end{aligned}
$$

This pair of functions is in the range of $D G(\Omega, 0,0)$ if and only if the vector $e_{m} \in \mathbb{R}^{2}$ is a scalar multiple of one column of the matrix $M_{m}^{\alpha}$ seen in (6.4), which happens if and only if

$$
\left(\Omega+b^{-\alpha} \Theta_{m}-\Lambda_{1}(b)\right)^{2}-b^{2} \Lambda_{m}^{2}(b)=0 .
$$

Combining this equation with $\operatorname{det} M_{m}^{\alpha}=0$ we get

$$
\left(\Omega-\Theta_{m}+b^{2} \Lambda_{1}(b)\right)\left(\Omega+b^{-\alpha} \Theta_{m}-\Lambda_{1}(b)\right)+\left(\Omega+b^{-\alpha} \Theta_{m}-\Lambda_{1}(b)\right)^{2}=0 .
$$

This yields

$$
\left(\Omega+b^{-\alpha} \Theta_{m}-\Lambda_{1}(b)\right)\left[2 \Omega+\left(b^{2}-1\right) \Lambda_{1}(b)+\left(b^{-\alpha}-1\right) \Theta_{m}\right]=0
$$


which is equivalent to

$$
\Omega+b^{-\alpha} \Theta_{m}-\Lambda_{1}(b)=0 \quad \text { or } \quad \Omega=\frac{1}{2}\left(\left(1-b^{2}\right) \Lambda_{1}(b)+\left(1-b^{-\alpha}\right) \Theta_{m}\right) .
$$

This first possibility is excluded by (6.14) because $\Lambda_{m}(b) \neq 0$ and the second one is also impossible because it corresponds to a multiple eigenvalue which is not the case here. This concludes the proof of Proposition 6.1.

\section{Numerical StUdy OF $V$-STATES}

Even if there is a number of references on the numerical obtention of rotating $V$-states for the vortex patch problem (see for instance [16] and [17], and more recently [23]), up to our knowledge nothing similar has been done for the quasi-geostrophic problem. Therefore, for the sake of completeness, we will discuss in this section the numerical obtention of $V$-states for the quasi-geostrophic problem in both the simply-connected case and the doubly-connected case. Since the procedure is very similar to that in the vortex patch problem, we will omit some details, which can be consulted in [23].

7.1. Simply-connected $V$-states. We gather the main theoretical arguments from [20]. Given a simply-connected domain $D$ with boundary $z(\theta)$, where $\theta \in[0,2 \pi)$ is the Lagrangian parameter, and $z$ is counterclockwise parameterized, the contour dynamics equation for the quasi-geostrophic problem is

$$
z_{t}(\theta, t)=\frac{C_{\alpha}}{2 \pi} \int_{0}^{2 \pi} \frac{z_{\phi}(\phi, t) d \phi}{|z(\phi, t)-z(\theta, t)|^{\alpha}} ;
$$

from now on, in order not to burden the notation, we will not indicate explicitly the dependence on $t$.

The simply-connected domain $D$ is a $V$-state rotating with constant angular velocity $\Omega$, if and only if its boundary satisfies the following equation:

$$
\operatorname{Re}\left[\left(\Omega z(\theta)-\frac{C_{\alpha}}{2 \pi i} \int_{0}^{2 \pi} \frac{z_{\phi}(\phi) d \phi}{|z(\phi)-z(\theta)|^{\alpha}}\right) \overline{z_{\theta}(\theta)}\right]=0 .
$$

However, it is convenient to rewrite (7.2) in the following equivalent form:

$$
\operatorname{Re}\left[\left(\Omega z(\theta)-\frac{C_{\alpha}}{2 \pi i} \int_{0}^{2 \pi} \frac{\left(z_{\phi}(\phi)-z_{\theta}(\theta)\right) d \phi}{|z(\phi)-z(\theta)|^{\alpha}}\right) \overline{z_{\theta}(\theta)}\right]=0 .
$$

We use a pseudo-spectral method to find $m$-fold $V$-states from (7.3). We discretize $\theta \in[0,2 \pi)$ in $N$ equally spaced nodes $\theta_{i}=2 \pi i / N, i=0,1, \ldots, N-1$. Observe that, although (7.2) and (7.3) are trivially equivalent, the addition of $z_{\theta}(\theta)$ in the numerator cancels the singularity in the denominator; indeed,

$$
\lim _{\phi \rightarrow \theta} \frac{z_{\phi}(\phi)-z_{\theta}(\theta)}{|z(\phi)-z(\theta)|^{\alpha}}=0
$$

Therefore, bearing in mind (7.4), we can evaluate numerically with spectral accuracy the integral in (7.3) at a node $\theta=\theta_{i}$, by means of the trapezoidal rule, provided that $N$ is large 
enough:

$$
\frac{1}{2 \pi} \int_{0}^{2 \pi} \frac{\left(z_{\phi}(\phi)-z_{\theta}\left(\theta_{i}\right)\right) d \phi}{\left|z(\phi)-z\left(\theta_{i}\right)\right|^{\alpha}} \approx \frac{1}{N} \sum_{\substack{j=0 \\ j \neq i}}^{N-1} \frac{z_{\phi}\left(\phi_{j}\right)-z_{\theta}\left(\theta_{i}\right)}{\left|z\left(\phi_{j}\right)-z\left(\theta_{i}\right)\right|^{\alpha}}
$$

Now, in order to obtain $m$-fold $V$-states, we approximate the boundary $z$ as

$$
z(\theta)=e^{i \theta}\left[1+\sum_{k=1}^{M} a_{k} \cos (m k \theta)\right], \quad \theta \in[0,2 \pi)
$$

where the mean radius is 1 ; and we are imposing that $z(-\theta)=\overline{z(\theta)}$, i.e., we are looking for $V$-states symmetric with respect to the $x$-axis. For sampling purposes, $N$ has to be chosen such that $N \geq 2 m M+1$; additionally, it is convenient to take $N$ a multiple of $m$, in order to be able to reduce the $N$-element discrete Fourier transforms to $N / m$-element discrete Fourier transforms. If we write $N=m 2^{r}$, then $M=\left\lfloor\left(m 2^{r}-1\right) /(2 m)\right\rfloor=2^{r-1}-1$.

We introduce (7.6) into (7.3), and approximate the error in that equation by an $M$-term sine expansion:

$$
\operatorname{Re}\left[\left(\Omega z(\theta)-\frac{C_{\alpha}}{2 \pi i} \int_{0}^{2 \pi} \frac{\left(z_{\phi}(\phi)-z_{\theta}(\theta)\right) d \phi}{|z(\phi)-z(\theta)|^{\alpha}}\right) \overline{z_{\theta}(\theta)}\right]=\sum_{k=1}^{M} b_{k} \sin (m k \theta) .
$$

This last expression can be represented in a very compact way as

$$
\mathcal{F}_{\alpha, \Omega}\left(a_{1}, \ldots, a_{M}\right)=\left(b_{1}, \ldots, b_{M}\right),
$$

for a certain $\mathcal{F}_{\alpha, \Omega}: \mathbb{R}^{M} \rightarrow \mathbb{R}^{M}$.

Remark that, for any value of the parameters $\alpha$ and $\Omega$, we have trivially $\mathcal{F}_{\alpha, \Omega}(\mathbf{0})=\mathbf{0}$, i.e., the unit circumference is a solution of the problem. Therefore, the obtention of a simplyconnected $V$-state is reduced to finding numerically a nontrivial root $\left(a_{1}, \ldots, a_{M}\right)$ of $(7.8)$. To do so, we discretize the $(M \times M)$-dimensional Jacobian matrix $\mathcal{J}$ of $\mathcal{F}_{\alpha, \Omega}$ using first-order approximations. Fixed $|h| \ll 1$ (we have chosen $h=10^{-9}$ ), we have

$$
\frac{\partial}{\partial a_{1}} \mathcal{F}_{\alpha, \Omega}\left(a_{1}, \ldots, a_{M}\right) \approx \frac{\mathcal{F}_{\alpha, \Omega}\left(a_{1}+h, \ldots, a_{M}\right)-\mathcal{F}_{\alpha, \Omega}\left(a_{1}, \ldots, a_{M}\right)}{h} .
$$

Then, the sine expansion of (7.9) gives us the first row of $\mathcal{J}$, and so on. Hence, if the $n$-th iteration is denoted by $\left(a_{1}, \ldots, a_{M}\right)^{(n)}$, then the $(n+1)$-th iteration is given by

$$
\left(a_{1}, \ldots, a_{M}\right)^{(n+1)}=\left(a_{1}, \ldots, a_{M}\right)^{(n)}-\mathcal{F}_{\alpha, \Omega}\left(\left(a_{1}, \ldots, a_{M}\right)^{(n)}\right) \cdot\left[\mathcal{J}^{(n)}\right]^{-1},
$$

where $\left[\mathcal{J}^{(n)}\right]^{-1}$ denotes the inverse of the Jacobian matrix at $\left(a_{1}, \ldots, a_{M}\right)^{(n)}$. This iteration converges in a small number of steps to a nontrivial root for a large variety of initial data $\left(a_{1}, \ldots, a_{M}\right)^{(0)}$. In particular, it is usually enough to perturb the unit circumference by assigning a small value to $a_{1}{ }^{(0)}$, and leave the other coefficients equal to zero. Our stopping criterion is

$$
\max \left|\sum_{k=1}^{M} b_{k} \sin (m k \theta)\right|<t o l,
$$

where tol $=10^{-11}$. For the sake of coherence, we change eventually the sign of all the coefficients $\left\{a_{k}\right\}$, in order that, without loss of generality, $a_{1}>0$. 
Before moving forward, let us mention that it is possible to work numerically with other parametrizations than (7.6), like for example

$$
z(\theta)=e^{i \theta}\left(1+\sum_{k=1}^{M} a_{k} e^{-i m k \theta}\right), \quad \theta \in[0,2 \pi),
$$

where we have used a conformal mapping. However, a caveat should be make here. Indeed, unlike in the vortex patch problem, given a $V$-state $(z, \Omega)$, and $\mu>0,(\mu z, \Omega)$ is no longer a $V$-state, but, from (7.3), $\left(\mu z, \mu^{-\alpha} \Omega\right)$ is. Therefore, since we bifurcate from the unit circumference at a certain angular velocity $\Omega=\Omega_{m}^{\alpha}$, we always obtain, by uniqueness, the same $V$-states up to a scaling that implies also a modification on $\Omega$, irrespectively of the chosen numerical representation of $z$. An equivalent observation can be done for the doubly-connected case, etc.

7.1.1. Numerical experiments. According to Theorem 1 in [20], for fixed $\alpha \in(0,1)$ and $m \geq 2$, there is a family of $m$-fold $V$-states bifurcating from the unit circumference at the angular velocity

$$
\Omega_{m}^{\alpha} \triangleq \frac{\Gamma(1-\alpha)}{2^{1-\alpha} \Gamma^{2}\left(1-\frac{\alpha}{2}\right)}\left(\frac{\Gamma\left(1+\frac{\alpha}{2}\right)}{\Gamma\left(2-\frac{\alpha}{2}\right)}-\frac{\Gamma\left(m+\frac{\alpha}{2}\right)}{\Gamma\left(m+1-\frac{\alpha}{2}\right)}\right) .
$$

Given an $\Omega$ slightly smaller than $\Omega_{m}^{\alpha}$, it is straightforward to obtain the corresponding $V$ state with the technique described above. Then, we can use that $V$-state as a new initial datum to obtain another $V$-state with smaller $\Omega$, and so on. However, it seems impossible to obtain numerically $V$-states for $\Omega$ strictly larger than $\Omega_{m}^{\alpha}$. This means that the bifurcation is pitchfork and this fact follows from a symmetry argument: if $(\Omega, z)$ is a solution of $(7.2)$ then $(\Omega,-z)$ is a solution too.

Bearing in mind (7.13), we are able to obtain $V$-states for an arbitrary large number of symmetries $m$. For instance, in Figure 1, we have plotted simultaneously the $V$-states corresponding to $\alpha=0.5, m=10$, for $\Omega=0.5592$ and $\Omega=0.556,0.552, \ldots, 0.528$. In all the numerical experiments in this section, we take $N=256 \times m$ nodes. Since, according to (7.13), $\Omega_{10}^{0.5}=0.559238 \ldots$, the $V$-state corresponding to $\Omega=0.5592$, in black, is practically a circumference, as expected. On the other hand, the $V$-state corresponding to $\Omega=0.528$ is plotted in red. Observe that we have been unable to obtain the $V$-state corresponding to, say, $\Omega=0.527$; this makes us wonder whether the $V$-state in red might be close from developing some kind of singularity.

It is an established fact that simply-connected limiting $V$-states do exist for $\alpha=0$, which corresponds to the vortex patch problem. These $V$-states are obtained after bifurcating from the circumference at $\Omega_{m} \triangleq(m-1) /(2 m)$, which corresponds to $(7.13)$ evaluated at $\alpha=0$, and decreasing $\Omega$ as much as possible, until corner-shaped singularities appear. Furthermore, it has been proved in $[37,45]$ that the angle at the corners is always $\pi / 2$, irrespectively of the number $m$ of symmetries. Therefore, we are interested in understanding what happens when $\alpha>0$.

In Figure 2, we have plotted $V$-states corresponding to $m=3,4,5,6,7$; for the vortex patch problem, and for $\alpha=0.1, \alpha=0.5$, and $\alpha=0.9$ (i.e, a value of $\alpha$ rather close to zero, an intermediate value, and a value rather close to one). We have used the smallest possible (four-digit) values of $\Omega$, which are offered in Table 1 , in such a way that the experiments 


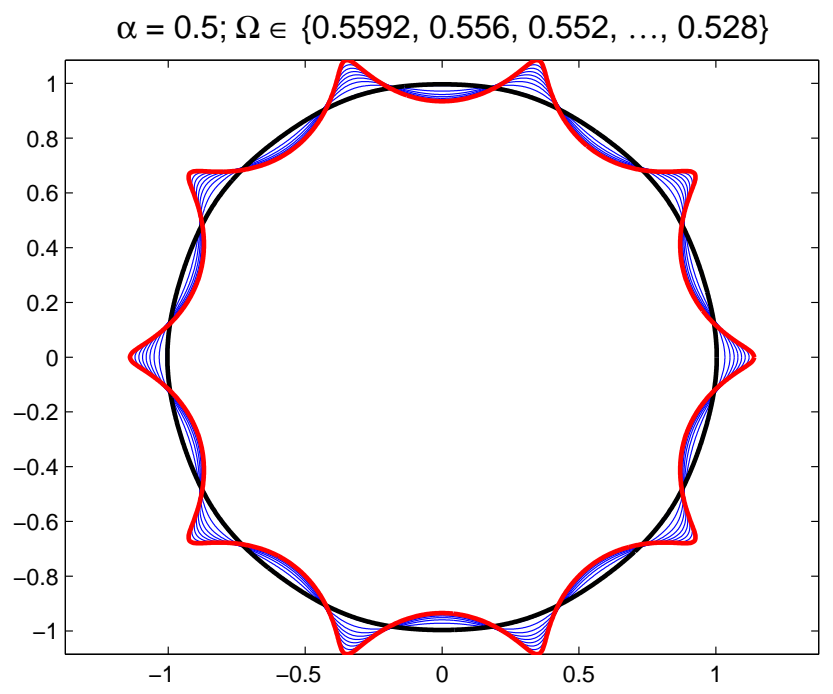

FiguRE 1. 10-fold $V$-states corresponding to $\alpha=0.5$, for different values of $\Omega$.

become numerically instable for smaller values of $\Omega$. In order to facilitate the comparison between different $m$, we have plotted $\left.z(\theta) / \max _{\theta}(|z(\theta)|)\right)$, rather than $z(\theta)$.

\begin{tabular}{|c|c|c|c|c|c|}
\hline$\alpha / m$ & 3 & 4 & 5 & 6 & 7 \\
\hline 0 & 0.3013 & 0.3540 & 0.3842 & 0.4040 & 0.4180 \\
\hline 0.1 & 0.2965 & 0.3544 & 0.3884 & 0.4112 & 0.4275 \\
\hline 0.5 & 0.2690 & 0.3498 & 0.4021 & 0.4399 & 0.4689 \\
\hline 0.9 & 0.2191 & 0.3202 & 0.3918 & 0.4476 & 0.4933 \\
\hline
\end{tabular}

TABle 1. Values of $\Omega$ for the $V$-states plotted in Figure 2. The case $\alpha=0$ corresponds to the vortex patch problem.

Figure 2 confirms graphically that the angles developed by the five limiting $V$-states in the vortex patch problem are identical. On the other hand, when $\alpha=0.1$, the $V$-states depicted are very similar to the limiting $V$-states in the vortex patch problem, whereas, for $\alpha=0.5$, and especially for $\alpha=0.9$, it is unclear whether any singularity has happened at all. In order to shed some light on this, we have plotted in Figure 3 the respective bifurcation diagrams of $a_{1}$ in (7.6) with respect to $\Omega$. In the vortex patch problem, we have a family of monotonic curves already shown in [41]. When $\alpha=0.1$, the curves are very similar to those in the vortex patch problem, but slightly bigger and more spaced. Then, as $\alpha$ grows, the curves become bigger and bigger, and more and more spaced. Furthermore, when $\alpha=0.9$, the curves are partially superposed; for example, there are 2 -fold and 3 -fold $V$-states with the same $\Omega$. This phenomenon also happens in the last four curves, when $\alpha=0.5$. However, the most striking fact from Figure 3 is that all the fifteen curves, corresponding to $\alpha=0.1, \alpha=0.5$ and $\alpha=0.9$, lose monotonicity at their left ends. In fact, especially for $\alpha=0.9$, incipient hooks are clearly visible. This seems to suggest the presence of saddle-node bifurcation points (see for instance [28]) at a certain $\Omega=\Omega_{c}$, which is indeed the case, as we will show in the following lines. 

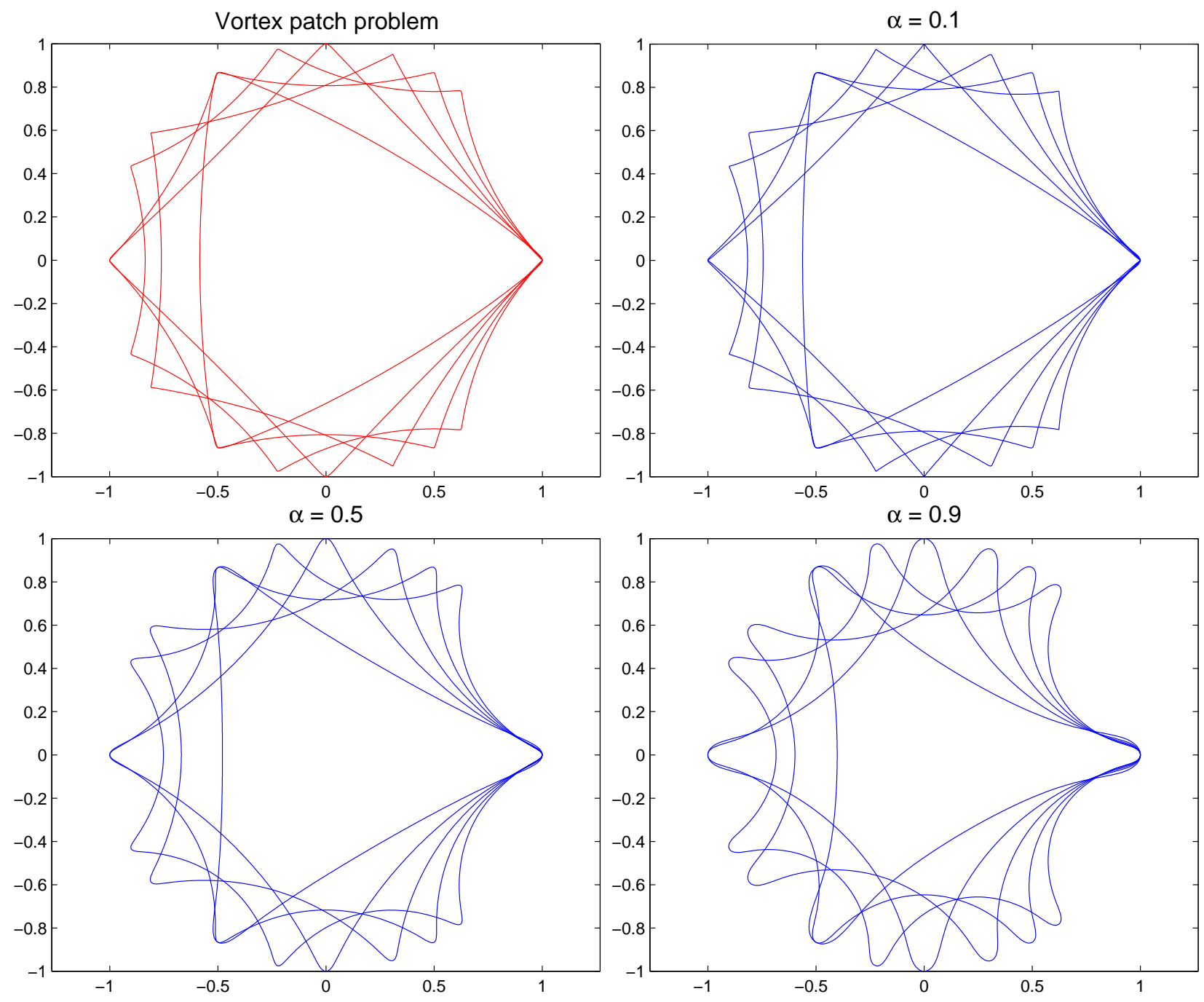

FiguRE 2. $V$-states corresponding to the vortex patch problem (i.e., $\alpha=0$ ), and to $\alpha=0.1, \alpha=0.5$, and $\alpha=0.9$; with $m=3,4,5,6,7$. To facilitate the comparison between different $m$, we have plotted $z(\theta) / \max _{\theta}(|z(\theta)|)$, instead of $z(\theta)$. We have chosen the smallest possible (four-digit) values of $\Omega$, which are offered in Table 1.

We work with the bifurcation curve corresponding to $\alpha=0.9$ and $m=3$ in Figure 3, because it has the most pronounced hook, but everything that follows is applicable to the other bifurcation curves as well. We need to estimate the corresponding $\Omega_{c}$ with enough accuracy. In our case, we have taken $\Omega_{c}=0.21904$. Then, given $0<\epsilon \ll 1$ (we have taken here $\left.\epsilon=10^{-4}\right)$, we calculate the $V$-states corresponding to $\Omega^{(A)} \equiv \Omega_{c}+4 \epsilon, \Omega^{(B)} \equiv \Omega_{c}+3 \epsilon$, $\Omega^{(C)} \equiv \Omega_{c}+2 \epsilon, \Omega^{(D)} \equiv \Omega_{c}+\epsilon$, and $\Omega^{(E)} \equiv \Omega_{c}$, whose coefficients in (7.6) are respectively denoted as $\left\{a_{k}^{(A)}\right\},\left\{a_{k}^{(B)}\right\},\left\{a_{k}^{(C)}\right\},\left\{a_{k}^{(D)}\right\}$, and $\left\{a_{k}^{(E)}\right\}$. The main idea is to introduce a new parameter $\lambda$, instead of $\Omega$, in such a way that $\lambda=\lambda^{(A)}$ corresponds to $\Omega=\Omega^{(A)}$, and so on. We set $\lambda^{(A)}=0, \lambda^{(B)}=\lambda^{(A)}+\left[\left(\Omega^{(B)}-\Omega^{(A)}\right)^{2}+\left(a_{1}^{(B)}-a_{1}^{(A)}\right)^{2}\right]^{1 / 2}, \lambda^{(C)}=$ $\lambda^{(B)}+\left[\left(\Omega^{(C)}-\Omega^{(B)}\right)^{2}+\left(a_{1}^{(C)}-a_{1}^{(B)}\right)^{2}\right]^{1 / 2}, \lambda^{(D)}=\lambda^{(C)}+\left[\left(\Omega^{(D)}-\Omega^{(C)}\right)^{2}+\left(a_{1}^{(D)}-a_{1}^{(C)}\right)^{2}\right]^{1 / 2}$, $\lambda^{(E)}=\lambda^{(D)}+\left[\left(\Omega^{(E)}-\Omega^{(D)}\right)^{2}+\left(a_{1}^{(E)}-a_{1}^{(D)}\right)^{2}\right]^{1 / 2}$. Therefore, our problem is reduced to finding 

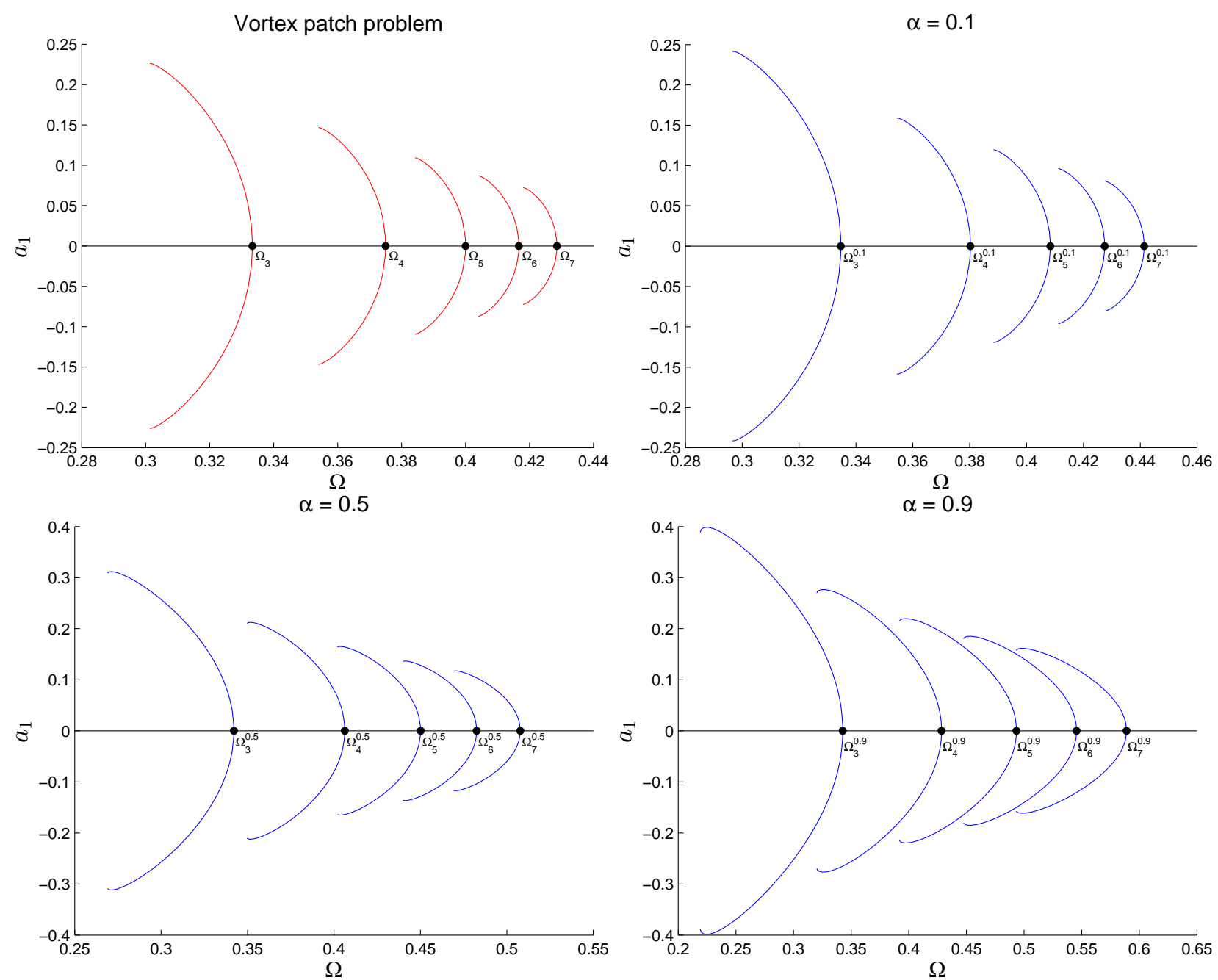

FiguRE 3. Bifurcations diagrams, for the vortex patch problem (i.e., $\alpha=0$ ), and for $\alpha=0.1, \alpha=0.5$, and $\alpha=0.9$; with $m=3,4,5,6,7$.

the $V$-state corresponding to some $\lambda$ slightly larger than $\lambda^{(E)}$; and a fairly good initial guess for that $V$-state can be obtained by means of a four-degree Lagrange interpolation polynomial. More precisely, let us define

$$
c^{(A)}=\frac{\left(\lambda-\lambda^{(B)}\right)\left(\lambda-\lambda^{(C)}\right)\left(\lambda-\lambda^{(D)}\right)\left(\lambda-\lambda^{(E)}\right)}{\left(\lambda^{(A)}-\lambda^{(B)}\right)\left(\lambda^{(A)}-\lambda^{(C)}\right)\left(\lambda^{(A)}-\lambda^{(D)}\right)\left(\lambda^{(A)}-\lambda^{(E)}\right)},
$$

and, in a similar way, $c^{(B)}, c^{(C)}, c^{(D)}$, and $c^{(E)}$. Then,

$$
\begin{aligned}
\Omega(\lambda) & =c^{(A)} \Omega^{(A)}+c^{(B)} \Omega^{(B)}+c^{(C)} \Omega^{(C)}+c^{(D)} \Omega^{(D)}+c^{(E)} \Omega^{(E)}, \\
a_{k}(\lambda) & =c^{(A)} a_{k}^{(A)}+c^{(B)} a_{k}^{(B)}+c^{(C)} a_{k}^{(C)}+a_{k} \Omega^{(D)}+a_{k} \Omega^{(E)}, \quad k=1, \ldots, M .
\end{aligned}
$$

Remark that a couple of trials may be needed until a good choice of $\Omega^{(A)}, \ldots$, and of $\lambda$ is found, i.e., values that enable us to continue the bifurcation curve, and not to come back to some already known $V$-state. In our case, we have chosen $\lambda$ equal to $\lambda^{(E)}$ plus the mean of 
the four previous increments of $\lambda$, i.e.,

$$
\begin{aligned}
\lambda & =\lambda^{(E)}+\frac{\left(\lambda^{(E)}-\lambda^{(D)}\right)+\left(\lambda^{(D)}-\lambda^{(C)}\right)+\left(\lambda^{(C)}-\lambda^{(B)}\right)+\left(\lambda^{(B)}-\lambda^{(A)}\right)}{4} \\
& =\frac{5 \lambda^{(E)}-\lambda^{(A)}}{4} .
\end{aligned}
$$

After applying this technique just once, we have successfully obtained a $V$-state corresponding to $\Omega=0.219054 \ldots$... i.e., a $V$-state beyond the critical point. It may be useful (and sometimes even convenient) to iterate several times the procedure, after updating $\lambda^{(A)}=\lambda^{(B)}$, $\lambda^{(B)}=\lambda^{(C)}, \lambda^{(C)}=\lambda^{(C)}, \lambda^{(C)}=\lambda^{(D)}$, and $\lambda^{(E)}=\lambda$. In fact, it can be even applied from the very beginning, to obtain all the bifurcation curves in 3 in their integrity, with an important spare of computational time. In Figure 4, we plot on the left-hand side the completed bifurcation curve until $\Omega=0.236$; the piece of curve beyond the saddle-node bifurcation point, absent in Figure 3, is shown in thicker stroke.

It is possible to still continue the bifurcation curve, although the results are to be taken with prudence, because higher spatial resolution is needed. Further numerical experiments, which include the use of alternative parameterizations of $z$, would suggest the eventual formation of cusp-shaped singularities at the corners. They would also suggest the presence of additional saddle-node bifurcation points, in such a way that the bifurcation curve in 4 would show spiral-like structures at its ends. Nevertheless, since our results are still inconclusive, we postpone this challenging issue for the future.
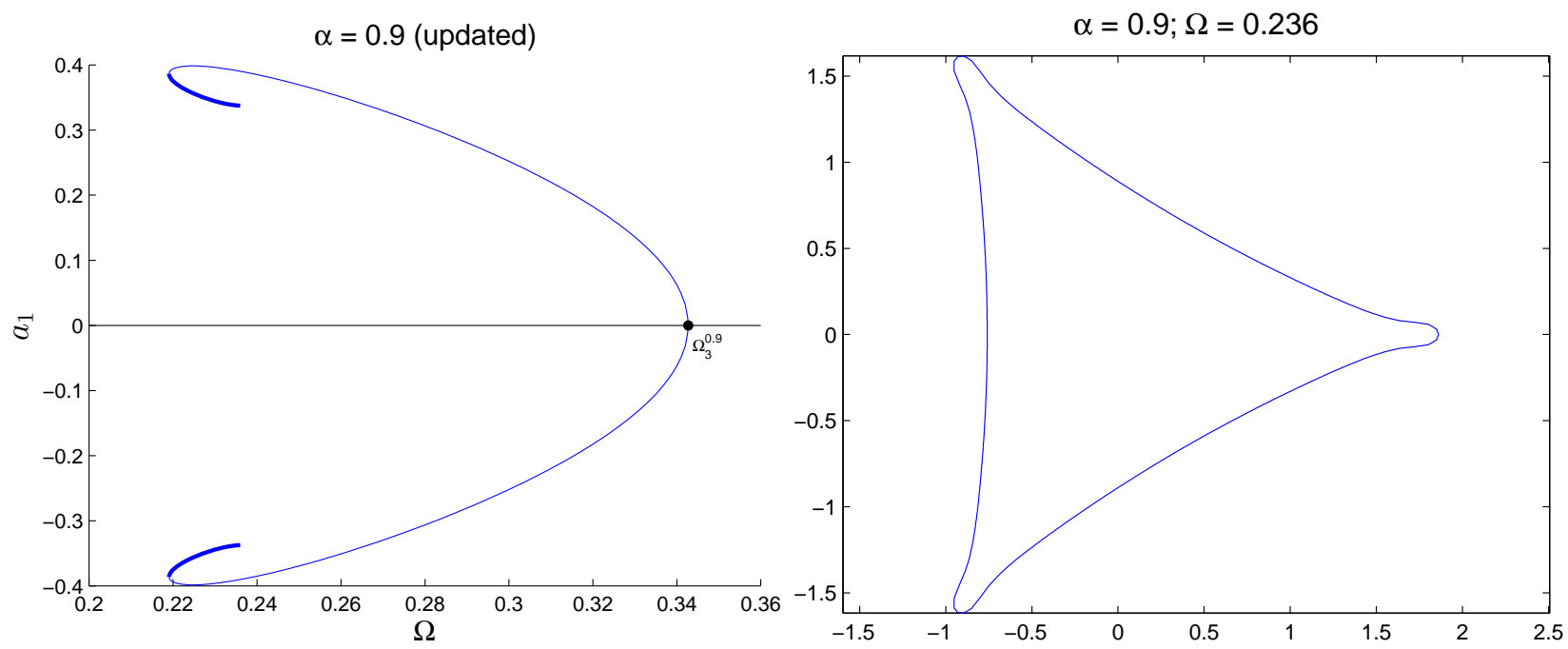

FiguRE 4. Left: Extended bifurcation curve, for $m=3$, and $\alpha=0.9$. Right: $V$-state beyond the saddle-node bifurcation point.

On the other hand, based on the previous pages and on additional numerical experiments that we have carried on, we conjecture the existence of saddle-node bifurcation points for all $m \geq 3$ and for all $\alpha \in(0,1)$. However, as $\alpha$ decreases, smaller and smaller structures are expected at the ends of the bifurcation curves, until $\alpha=0$, when they disappear. Indeed, in the vortex patch problem, as mentioned above, the bifurcation curves are always monotonic. 
We cannot finish this section, without saying something about the case $m=2$, which has a pretty different behavior and is interesting per se. In Figure 5, we have plotted 2fold $V$-states for $\alpha=0.01$ and different values of $\Omega$, starting from $\Omega=0.2496$, which is close to $\Omega_{2}^{0.01}=0.249667 \ldots$, so the corresponding $V$-state, in black, is practically a unit circumference. Since $\alpha$ is small, we might expect to have a similar behavior to that in the vortex patch problem, where the $V$-states tend to degenerate to a segment as $\Omega$ decreases. However, although this is true for $\Omega$ close enough to $\Omega_{2}^{0.01}$, there is an instant when convexity is lost, and the $V$-states get a more and more pronounced $\infty$-shape, as $\Omega$ decreases. Remark that $\Omega$ cannot be smaller than a certain value, which corresponds approximately to $\Omega=0.1057$, and whose corresponding $V$-state is plotted in red. Let us mention that the situation is very similar for greater $\alpha$, even for those close to 1 .

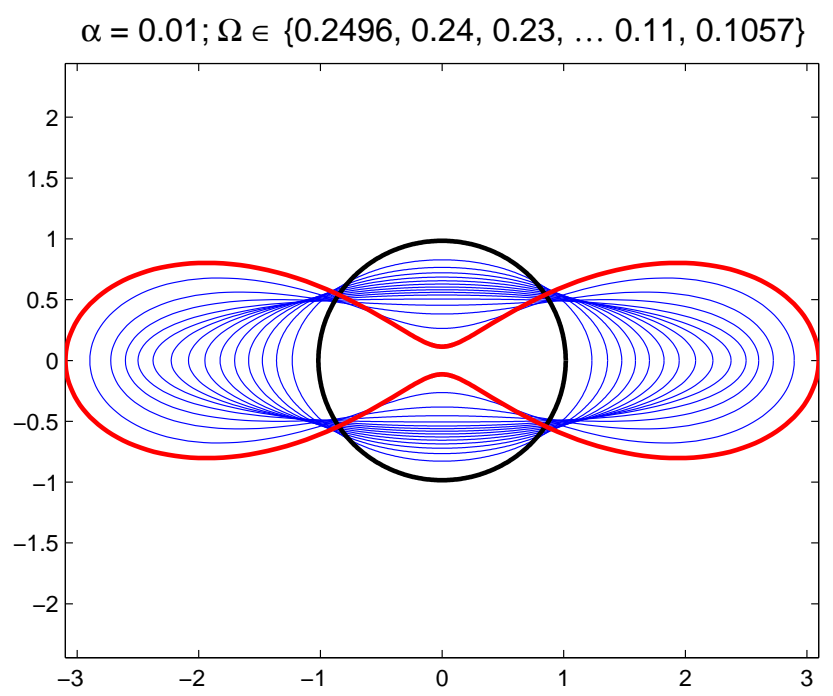

FiguRE 5. 2-fold $V$-states corresponding to $\alpha=0.2$, for different values of $\Omega$.

In Figure 5, the $V$-state in red seems to have developed no singularity. Again, insight into what is happening is given by the bifurcation curve of $a_{1}$ in (7.6) with respect to $\Omega$, for $\alpha=0.01$, which is plotted in Figure (7.6). In that figure, we have also plotted the bifurcation curves for $\alpha=0.1,0.2,0.3$, being the four curves very similar to each other.

As with $m \geq 3$, the bifurcation curves suggest the existence of saddle-node bifurcation points. To see whether this is indeed the case, we have used the previously described continuation method for $\alpha=0.01$, taking $\Omega_{c}=0.10567$, and $\epsilon=10^{-4}$. Figure 7 confirms our suspicions. On the left-hand side, we plot the completed bifurcation curve until $\Omega=0.1061$; the piece of curve beyond the saddle-node bifurcation point, absent in Figure 6, is shown in thicker stroke. Remark that, unlike Figure 4, a zoom is necessary in order for the hook to be appreciated. On the right-hand side, we plot the new $V$-state corresponding to a slightly larger $\Omega$, i.e., $\Omega=0.1061407$ (and such that $\Omega=0.1061408$ is unstable), with twice as many nodes, i.e, $N=512 \times 2$. Apparently, a self-intersection has happened, although a powerful zoom shows that the distance between the two inner pieces of curve is approximately $5.9 \times 10^{-3}$; moreover, there are apparently enough nodes in that region, so it seems that we could decrease that distance even further, by increasing the eight decimal of $\Omega$, and so on. Even if we rather 


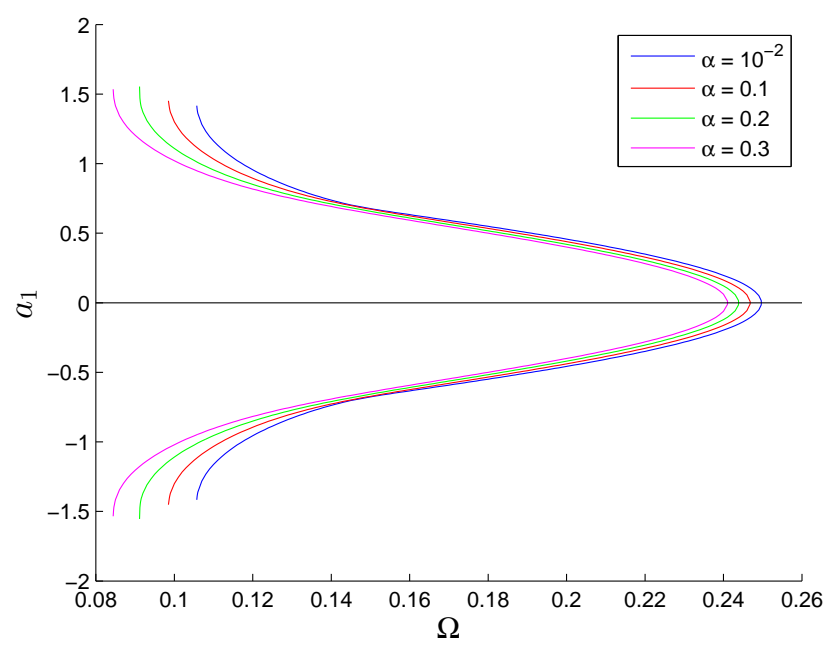

Figure 6. Bifurcation curves, for $m=2$, and $\alpha=0.01,0.1,0.2,0.3$. We plot the value of the first coefficient $a_{1}$ in (7.6) with respect to $\Omega$. The right-most curve corresponds to the smallest $\alpha$, and so on.

think that a self-intersection will eventually occur (see [9] and [33] for similar phenomena in the vortex patch problem), further study is required here.
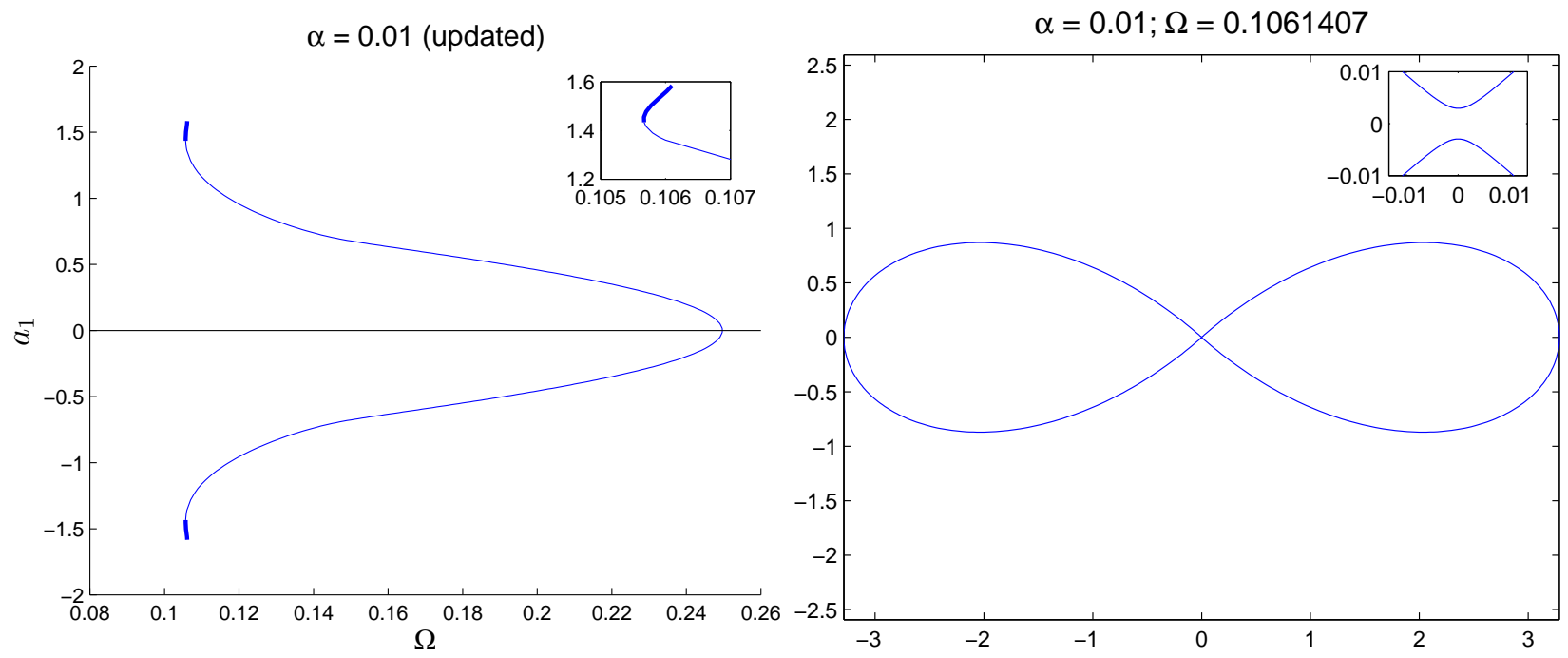

FiguRE 7. Left: Extended bifurcation curve, for $m=2$, and $\alpha=0.01$. Right: $V$-state beyond the saddle-node bifurcation point. A self-intersection has almost occurred.

7.2. Doubly-connected $V$-states. Given a doubly-connected domain $D$ with outer boundary $z_{1}(\theta)$ and inner boundary $z_{2}(\theta)$, where $\theta \in[0,2 \pi)$ is the Lagrangian parameter, and $z_{1}$ 
and $z_{2}$ are counterclockwise parameterized, the contour dynamics equations for the quasigeostrophic problem are

$$
\begin{aligned}
& z_{1, t}(\theta, t)=\frac{C_{\alpha}}{2 \pi} \int_{0}^{2 \pi} \frac{z_{1, \phi}(\phi, t) d \phi}{\left|z_{1}(\phi, t)-z_{1}(\theta, t)\right|^{\alpha}}-\frac{C_{\alpha}}{2 \pi} \int_{0}^{2 \pi} \frac{z_{2, \phi}(\phi, t) d \phi}{\left|z_{2}(\phi, t)-z_{1}(\theta, t)\right|^{\alpha}}, \\
& z_{2, t}(\theta, t)=\frac{C_{\alpha}}{2 \pi} \int_{0}^{2 \pi} \frac{z_{1, \phi}(\phi, t) d \phi}{\left|z_{1}(\phi, t)-z_{2}(\theta, t)\right|^{\alpha}}-\frac{C_{\alpha}}{2 \pi} \int_{0}^{2 \pi} \frac{z_{2, \phi}(\phi, t) d \phi}{\left|z_{2}(\phi, t)-z_{2}(\theta, t)\right|^{\alpha}}
\end{aligned}
$$

where, as in the simply-connected case, we will omit the explicit dependence on $t$.

The doubly-connected domain $D$ is a $V$-state if and only if its boundaries satisfy the following equations:

$$
\begin{aligned}
& \operatorname{Re}\left[\left(\Omega z_{1}(\theta)-\frac{C_{\alpha}}{2 \pi i} \int_{0}^{2 \pi} \frac{z_{1, \phi}(\phi) d \phi}{\left|z_{1}(\phi)-z_{1}(\theta)\right|^{\alpha}}+\frac{C_{\alpha}}{2 \pi i} \int_{0}^{2 \pi} \frac{z_{2, \phi}(\phi) d \phi}{\left|z_{2}(\phi)-z_{1}(\theta)\right|^{\alpha}}\right) \overline{z_{1, \theta}(\theta)}\right]=0 \\
& \operatorname{Re}\left[\left(\Omega z_{2}(\theta)-\frac{C_{\alpha}}{2 \pi i} \int_{0}^{2 \pi} \frac{z_{1, \phi}(\phi) d \phi}{\left|z_{1}(\phi)-z_{2}(\theta)\right|^{\alpha}}+\frac{C_{\alpha}}{2 \pi i} \int_{0}^{2 \pi} \frac{z_{2, \phi}(\phi) d \phi}{\left|z_{2}(\phi)-z_{2}(\theta)\right|^{\alpha}}\right) \overline{z_{2, \theta}(\theta)}\right]=0 .
\end{aligned}
$$

However, as we did in (7.3), it is convenient to rewrite them in the following equivalent form:

$$
\begin{aligned}
\operatorname{Re}\left[\left(\Omega z_{1}(\theta)\right.\right. & -\frac{C_{\alpha}}{2 \pi i} \int_{0}^{2 \pi} \frac{\left(z_{1, \phi}(\phi)-z_{1, \theta}(\theta)\right) d \phi}{\left|z_{1}(\phi)-z_{1}(\theta)\right|^{\alpha}} \\
& \left.\left.+\frac{C_{\alpha}}{2 \pi i} \int_{0}^{2 \pi} \frac{z_{2, \phi}(\phi) d \phi}{\left|z_{2}(\phi)-z_{1}(\theta)\right|^{\alpha}}\right) \overline{z_{1, \theta}(\theta)}\right]=0, \\
\operatorname{Re}\left[\left(\Omega z_{2}(\theta)\right.\right. & -\frac{C_{\alpha}}{2 \pi i} \int_{0}^{2 \pi} \frac{z_{1, \phi}(\phi) d \phi}{\left|z_{1}(\phi)-z_{2}(\theta)\right|^{\alpha}} \\
& \left.\left.+\frac{C_{\alpha}}{2 \pi i} \int_{0}^{2 \pi} \frac{\left(z_{2, \phi}(\phi)-z_{2, \theta}(\theta)\right) d \phi}{\left|z_{2}(\phi)-z_{2}(\theta)\right|^{\alpha}}\right) \overline{z_{2, \theta}(\theta)}\right]=0 .
\end{aligned}
$$

We use again a pseudo-spectral method to find $V$-states. We discretize $\theta \in[0,2 \pi)$ in $N$ equally spaced nodes $\theta_{i}=2 \pi i / N, i=0,1, \ldots, N-1$. Since $z_{1}$ and $z_{2}$ never intersect, the second integral in (7.21) and the first integral in (7.22) can be evaluated numerically with spectral accuracy at a node $\theta=\theta_{i}$ by means of the trapezoidal rule; e.g.,

$$
\frac{1}{2 \pi} \int_{0}^{2 \pi} \frac{z_{2, \phi}(\phi) d \phi}{\left|z_{2}(\phi)-z_{1}\left(\theta_{i}\right)\right|^{\alpha}} \approx \frac{1}{N} \sum_{j=0}^{N-1} \frac{z_{2, \phi}\left(\phi_{j}\right)}{\left|z_{2}\left(\phi_{j}\right)-z_{1}\left(\theta_{i}\right)\right|^{\alpha}}
$$

Likewise, the first integral in (7.21) and the second integral in (7.22) can also be evaluated with spectral accuracy, reasoning as in (7.4); e.g.,

$$
\frac{1}{2 \pi} \int_{0}^{2 \pi} \frac{\left(z_{1, \phi}(\phi)-z_{1, \theta}\left(\theta_{i}\right)\right) d \phi}{\left|z_{1}(\phi)-z_{1}\left(\theta_{i}\right)\right|^{\alpha}} \approx \frac{1}{N} \sum_{\substack{j=0 \\ j \neq i}}^{N-1} \frac{z_{1, \phi}\left(\phi_{j}\right)-z_{1, \theta}\left(\theta_{i}\right)}{\left|z_{1}\left(\phi_{j}\right)-z_{1}\left(\theta_{i}\right)\right|^{\alpha}}
$$

Therefore, for $N$ large enough, we can evaluate with spectral accuracy all the terms in (7.21)-(7.22) at $\theta=\theta_{i}$. 
In order to obtain doubly connected $m$-fold $V$-states, we approximate $z_{1}$ and $z_{2}$ as in (7.6):

$$
z_{1}(\theta)=e^{i \theta}\left[1+\sum_{k=1}^{M} a_{1, k} \cos (m k \theta)\right], \quad z_{2}(\theta)=e^{i \theta}\left[b+\sum_{k=1}^{M} a_{2, k} \cos (m k \theta)\right],
$$

where $\theta \in[0,2 \pi)$, the mean outer and inner radii are respectively 1 and $b$; and we are imposing that $z_{1}(-\theta)=\overline{z_{1}(\theta)}$ and $z_{2}(-\theta)=\overline{z_{2}(\theta)}$, i.e., we are looking for $V$-states symmetric with respect to the $x$-axis. Again, if we choose $N$ of the form $N=m 2^{r}$, then $M=$ $\left\lfloor\left(m 2^{r}-1\right) /(2 m)\right\rfloor=2^{r-1}-1$.

We introduce (7.25) into (7.21)-(7.22), and approximate the error in those equations by an $M$-term sine expansion:

$$
\begin{aligned}
\operatorname{Re}\left[\left(\Omega z_{1}(\theta)\right.\right. & -\frac{C_{\alpha}}{2 \pi i} \int_{0}^{2 \pi} \frac{\left(z_{1, \phi}(\phi)-z_{1, \theta}(\theta)\right) d \phi}{\left|z_{1}(\phi)-z_{1}(\theta)\right|^{\alpha}} \\
& \left.\left.+\frac{C_{\alpha}}{2 \pi i} \int_{0}^{2 \pi} \frac{z_{2, \phi}(\phi) d \phi}{\left|z_{2}(\phi)-z_{1}(\theta)\right|^{\alpha}}\right) \overline{z_{1, \theta}(\theta)}\right]=\sum_{k=1}^{M} b_{1, k} \sin (m k \theta), \\
\operatorname{Re}\left[\left(\Omega z_{2}(\theta)\right.\right. & -\frac{C_{\alpha}}{2 \pi i} \int_{0}^{2 \pi} \frac{z_{1, \phi}(\phi) d \phi}{\left|z_{1}(\phi)-z_{2}(\theta)\right|^{\alpha}} \\
& \left.\left.+\frac{C_{\alpha}}{2 \pi i} \int_{0}^{2 \pi} \frac{\left(z_{2, \phi}(\phi)-z_{2, \theta}(\theta)\right) d \phi}{\left|z_{2}(\phi)-z_{2}(\theta)\right|^{\alpha}}\right) \overline{z_{2, \theta}(\theta)}\right]=\sum_{k=1}^{M} b_{2, k} \sin (m k \theta) .
\end{aligned}
$$

As in (7.8), this last system of equations can be represented in a very compact way as

$$
\mathcal{F}_{b, \alpha, \Omega}\left(a_{1,1}, \ldots, a_{1, M}, a_{2,1}, \ldots, a_{2, M}\right)=\left(b_{1,1}, \ldots, b_{1, M}, b_{2,1}, \ldots, b_{2, M}\right),
$$

for a certain $\mathcal{F}_{\alpha, \Omega}: \mathbb{R}^{2 M} \rightarrow \mathbb{R}^{2 M}$.

Remark that, for any value of the parameters $b, \alpha$ and $\Omega$, we have trivially $\mathcal{F}_{b, \alpha, \Omega}(\mathbf{0})=$ 0, i.e., any circular annulus is a solution of the problem. Therefore, the obtention of a doubly-connected $V$-state is reduced to finding numerically $\left\{a_{1, k}\right\}$ and $\left\{a_{2, k}\right\}$, such that $\left(a_{1,1}, \ldots, a_{1, M}, a_{2,1}, \ldots, a_{2, M}\right)$ is a nontrivial root of (7.27). To do so, we discretize the $(2 M \times 2 M)$-dimensional Jacobian matrix $\mathcal{J}$ of $\mathcal{F}_{b, \alpha, \Omega}$ as in (7.9), taking $h=10^{-9}$ :

$$
\begin{aligned}
& \frac{\partial}{\partial a_{1,1}} \mathcal{F}_{b, \alpha, \Omega}\left(a_{1,1}, \ldots, a_{1, M}, a_{2,1}, \ldots, a_{2, M}\right) \\
& \quad \approx \frac{\mathcal{F}_{b, \alpha, \Omega}\left(a_{1,1}+h, a_{1,2}, \ldots, a_{1, M}, a_{2,1}, \ldots, a_{2, M}\right)-\mathcal{F}_{b, \alpha, \Omega}\left(a_{1,1}, \ldots, a_{1, M}, a_{2,1}, \ldots, a_{2, M}\right)}{h},
\end{aligned}
$$

Then, the sine expansion of (7.28) gives us the first row of $\mathcal{J}$, and so on. Hence, if the $n$-th iteration is denoted by $\left(a_{1,1}, \ldots, a_{1, M}, a_{2,1}, \ldots, a_{2, M}\right)^{(n)}$, then the $(n+1)$-th iteration is given by

$$
\begin{aligned}
& \left(a_{1,1}, \ldots, a_{1, M}, a_{2,1}, \ldots, a_{2, M}\right)^{(n+1)} \\
& \quad=\left(a_{1,1}, \ldots, a_{1, M}, a_{2,1}, \ldots, a_{2, M}\right)^{(n)}-\mathcal{F}_{b, \alpha, \Omega}\left(\left(a_{1,1}, \ldots, a_{1, M}, a_{2,1}, \ldots, a_{2, M}\right)^{(n)}\right) \cdot\left[\mathcal{J}^{(n)}\right]^{-1},
\end{aligned}
$$

where $\left[\mathcal{J}^{(n)}\right]^{-1}$ denotes the inverse of the Jacobian matrix at $\left(a_{1,1}, \ldots, a_{1, M}, a_{2,1}, \ldots, a_{2, M}\right)^{(n)}$. To make this iteration converge, it is usually enough to perturb the annulus by assigning a 
small value to $a_{1,1}{ }^{(0)}$ or $a_{2,1}{ }^{(0)}$, and leave the other coefficients equal to zero. Our stopping criterion is

$$
\max \left|\sum_{k=1}^{M} b_{1, k} \sin (m k \theta)\right|<\text { tol } \wedge \max \left|\sum_{k=1}^{M} b_{2, k} \sin (m k \theta)\right|<t o l,
$$

where tol $=10^{-11}$. As in the vortex patch problem, $a_{1,1} \cdot a_{2,1}<0$, so, for the sake of coherence, we change eventually the sign of all the coefficients $\left\{a_{1, k}\right\}$ and $\left\{a_{2, k}\right\}$, in order that, without loss of generality, $a_{1,1}>0$ and $a_{2,1}<0$.

7.2.1. Numerical experiments. As we have seen, the procedure to find doubly-connected $m$ fold $V$-states is very similar in the vortex patch and in the quasi-geostrophic problems. However, as evidenced in the simply-connected case, the numerical study of the $V$-states for the quasi-geostrophic problem reveals itself as a much richer task. Indeed, unlike in the vortex patch problem, where we had just two parameters $b$ and $\Omega$, we have now a third parameter $\alpha$. Furthermore, since (7.21) and (7.22) are not homogeneous, choosing the mean outer radius not to be equal to one, as we are doing, would introduce a fourth parameter. Therefore, we will limit ourselves here to exposing a few relevant facts.

Theorem 1.1 states that, for any $\alpha \in(0,1)$, the inner mean radius $b$ must be greater than $b_{0}$, which is the unique solution of the equation

$$
b^{2} \Lambda_{1}(b)-\Lambda_{1}(1)+\frac{1}{2}=0, \quad \text { with } \Lambda_{1}(1)=\frac{\Gamma(1-\alpha)}{2^{1-\alpha} \Gamma^{2}\left(1-\frac{\alpha}{2}\right)} \frac{\Gamma\left(1+\frac{\alpha}{2}\right)}{\Gamma\left(2-\frac{\alpha}{2}\right)} .
$$

This is an important difference with the vortex patch problem, where no lower bound for $b$ exists.

In order to obtain $b_{0}$ (and other relevant quantities), we need to compute $\Lambda_{n}(b)$ accurately:

$$
\begin{aligned}
\Lambda_{n}(b) & =\frac{\Gamma\left(\frac{\alpha}{2}\right)}{2^{1-\alpha} \Gamma\left(1-\frac{\alpha}{2}\right)} \frac{\left(\frac{\alpha}{2}\right)_{n}}{n !} b^{n-1} F\left(\frac{\alpha}{2}, n+\frac{\alpha}{2}, n+1, b^{2}\right) \\
& =\frac{b^{n-1} \Gamma\left(\frac{\alpha}{2}+n\right)}{2^{1-\alpha} \Gamma\left(1-\frac{\alpha}{2}\right) n !} F\left(\frac{\alpha}{2}, n+\frac{\alpha}{2}, n+1, b^{2}\right) .
\end{aligned}
$$

The hypergeometric function $F$ is commonly implemented in most scientific packages; for instance, in MATLABC), $F(a, b, c, z)$ can be evaluated by means of the command hypergeom( $[\mathrm{a}, \mathrm{b}], \mathrm{c}, \mathrm{z}$ ), so we can find the only value $b_{0}$ satisfying (7.31) efficiently and with the greatest possible accuracy, by means a simple bisection technique. In Figure 8, we have plotted $b_{0}$ against 200 different values of $\alpha$, i.e., $\alpha=10^{-4}, 10^{-3}$, and $\alpha=$ $0.005,0.01, \ldots, 0.995$. Observe that $b_{0}$ tends very quickly to 1 ; for example, $b_{0}(0.76)>0.99$. On the other hand, $b_{0}(0)=0$, which is coherent with the vortex patch problem, where there is no lower bound for $b$.

However, we have discovered in our numerical experiments that (7.31) is not sharp. Indeed, we have been able to find $V$-states with $b$ much smaller than $b_{0}$. In all the numerical experiments in this section, we take $N=64 \times m$ nodes. In Figure 9, we have plotted $V$ states corresponding to $\alpha=0.9$ and $b=0.2$, where $b_{0}(0.9)-1=\mathcal{O}\left(10^{-9}\right)$. On the left-hand side, we have started to bifurcate from $\Omega_{4}^{+}(0.2)=0.4077 \ldots$. Observe that $z_{2}$ is practically a circumference, for all $\Omega$. Moreover, the $V$-state corresponding to $\Omega=0.4076$, in black, is practically a circular annulus, whereas the outer boundary of the $V$-state corresponding to $\Omega=0.296$, in red, has a marked star shape. For $\Omega$ slightly smaller than $\Omega=0.296$, the 


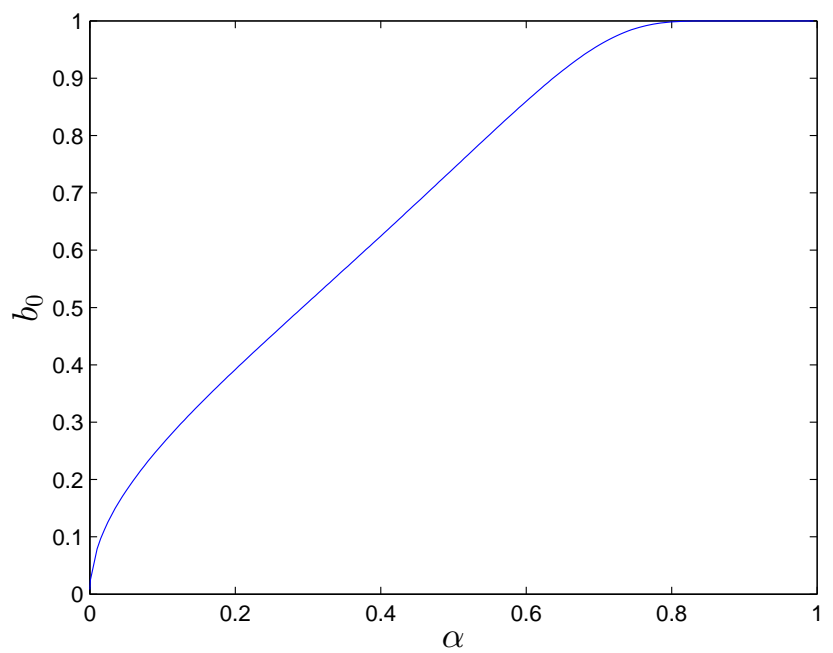

FiguRE 8. Unique solution of (7.31), $b_{0}$, in function of $\alpha$.

numerical experiments become instable. On the right-hand side, we have started to bifurcate from $\Omega_{4}^{-}(0.2)=-1.3055 \ldots$. The most remarkable fact is that $\Omega$ is always negative, i.e, the $V$-states rotate clockwise, which is an important difference with respect to the vortex patch problem. On the other hand, $z_{1}$ is practically a circumference, for all $\Omega$. The $V$-state corresponding to $\Omega=-1.305$, in black, is practically a circular annulus, whereas the inner boundary of the $V$-state corresponding to $\Omega=-0.849$, in red, has a marked star shape. For $\Omega$ slightly larger than $\Omega=-0.849$, the numerical experiments become instable.
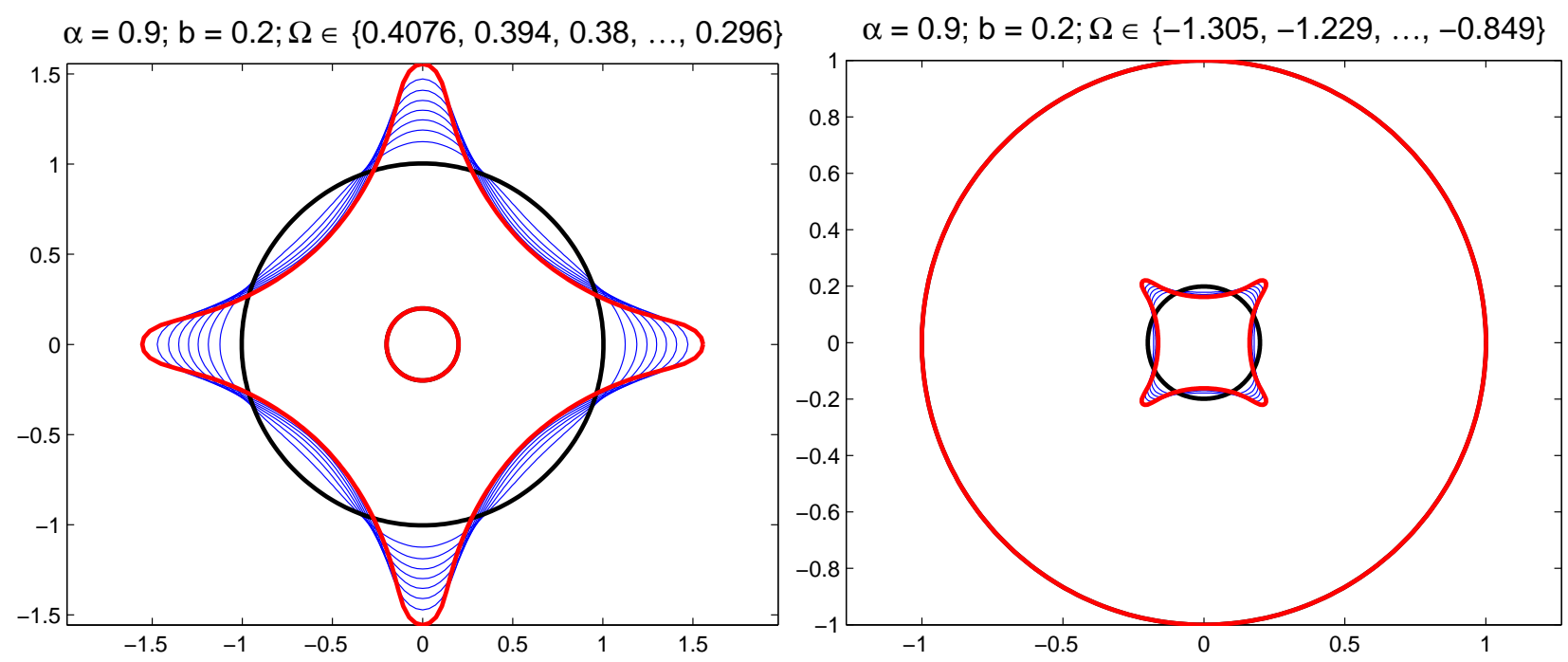

FIGURE 9. 4-fold $V$-states corresponding to $\alpha=0.9$, for different values of $\Omega$. On the left-hand side, we have started to bifurcate from $\Omega_{4}^{+}(0.2)=0.4077 \ldots$; on the right-hand side, from $\Omega_{4}^{-}(0.2)=-1.3055 \ldots$

Figure 9 shows the obvious parallelism with [23] in the vortex patch problem: as $b$ becomes smaller, bifurcating at $\Omega_{m}^{+}(b)$ yields doubly-connected $V$-states closer and closer to simplyconnected $V$-states; whereas, bifurcating at $\Omega_{m}^{-}(b)$ yields double-connected $V$-states closer 
and closer to the unit circumference. This explains why there are two bifurcation values of $\Omega$ in the doubly-connected case, and just one single bifurcation value of $\Omega$ in the simplyconnected case. Nonetheless, unlike what would have happened in the vortex patch problem, the $V$-states corresponding to $\Omega=0.296$ and to $\Omega=-0.849$ seem to have developed no singularity. Again, as in the simply-connected case, the explanation is given by the loss of monotonicity in the bifurcation curves of $a_{1,1}$ and $a_{2,1}$ in (7.25) with respect to $\Omega$, plotted in Figure 10, which predicts the existence of saddle-node bifurcation points. Observe also that, when we bifurcate from $\Omega_{4}^{+}(0.2), a_{1,1}$ clearly dominates; whereas, when we bifurcate from $\Omega_{4}^{-}(0.2), a_{2,1}$ dominates and $a_{1,1}$ is of the order of $\mathcal{O}\left(10^{-6}\right)$. This confirms our previous observations.
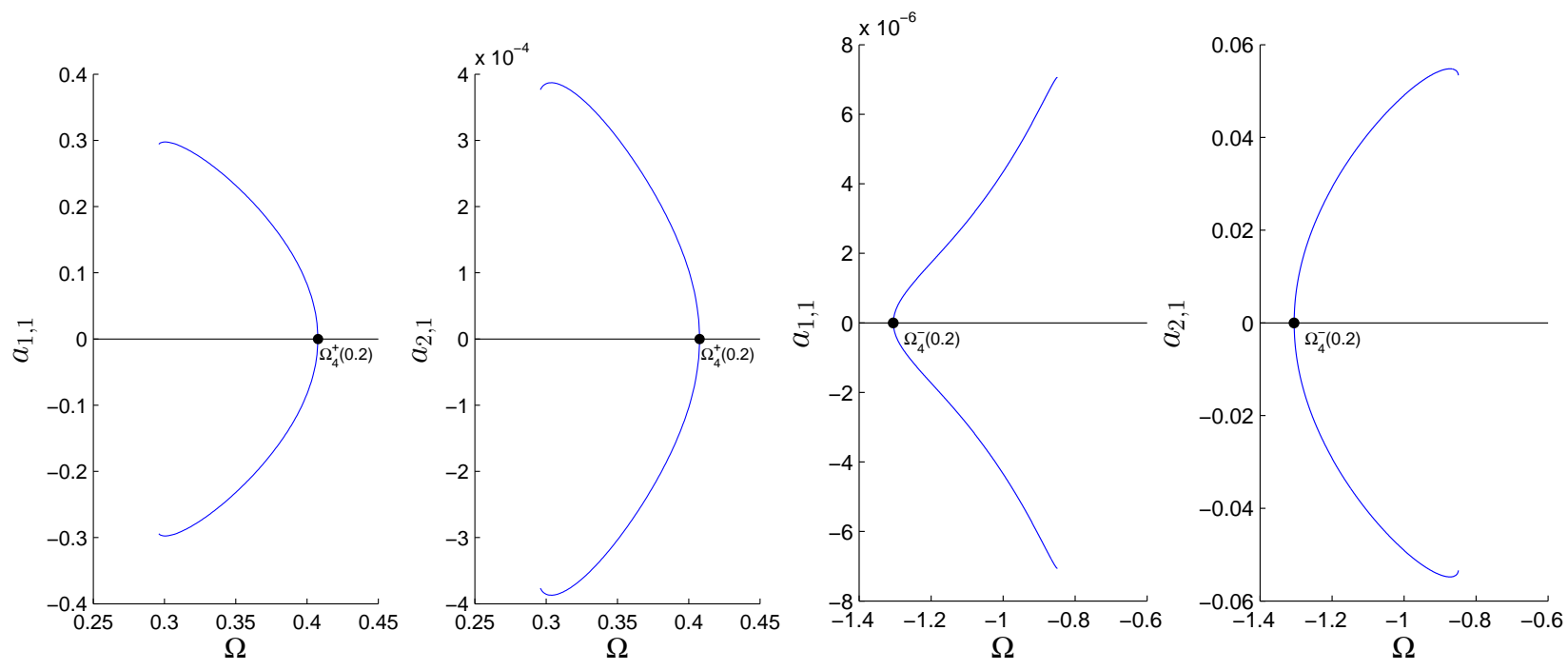

Figure 10. Bifurcation curves, for $m=4, \alpha=0.9$, and $b=0.2$. The leftmost two correspond to a bifurcation from $\Omega_{4}^{+}(0.2)$, whereas the rightmost two correspond to a bifurcation from $\Omega_{4}^{-}(0.2)$. Remember that $a_{1,1}$ and $a_{2,1}$ are such that $a_{1,1} \cdot a_{2,1}<0$.

Following the procedure explained in the simply-connected case (where we set $\lambda^{(A)}=0$, $\lambda^{(B)}=\lambda^{(A)}+\left[\left(\Omega^{(B)}-\Omega^{(A)}\right)^{2}+\left(a_{1,1}^{(B)}-a_{1,1}^{(A)}\right)^{2}+\left(a_{2,1}^{(B)}-a_{2,1}^{(A)}\right)^{2}\right]^{1 / 2}$, and so on $)$, we have continued the bifurcation curves at $\Omega=0.29507$, and $\Omega=-0.84878$, until $\Omega=0.3$ and $\Omega=-0.86$, respectively, as is shown in Figure 11. It is still possible to go a bit further, but, in order not to lose accuracy, a larger number of nodes is convenient. The pieces of curve beyond the saddle-node bifurcation points are shown in thicker stroke.

In Figure 12, we have plotted the $V$-states corresponding to $\Omega=3$ and $\Omega=-0.86$, but beyond the saddle-node bifurcation points. The differences with respect to the $V$-states in red from Figure 9 are evident. It would be interested to calculate which kind of limiting $V$ states develops. Finally, whether the lower bound restriction for $b$ can be ignored completely or not is another relevant question.

Another relevant fact is the possibility to find examples where $V$-states exist for all $\Omega \in$ $\left[\Omega_{m}^{-}, \Omega_{m}^{+}\right]$; such situation was also found in the vortex patch problem. We have considered, for instance, $m=4, \alpha=0.5$, and $b=0.65$, with $\Omega_{4}^{+}(0.65)=0.1480 \ldots$ and $\Omega_{4}^{-}(0.65)=$ $0.08168 \ldots$. Observe that $b_{0}(0.5)=0.7424 \ldots$, so we are violating the restriction on $b_{0}$ again. We have computed successfully all the $V$-states with $\Omega=0.082,0.083, \ldots, 0.148$. On the 

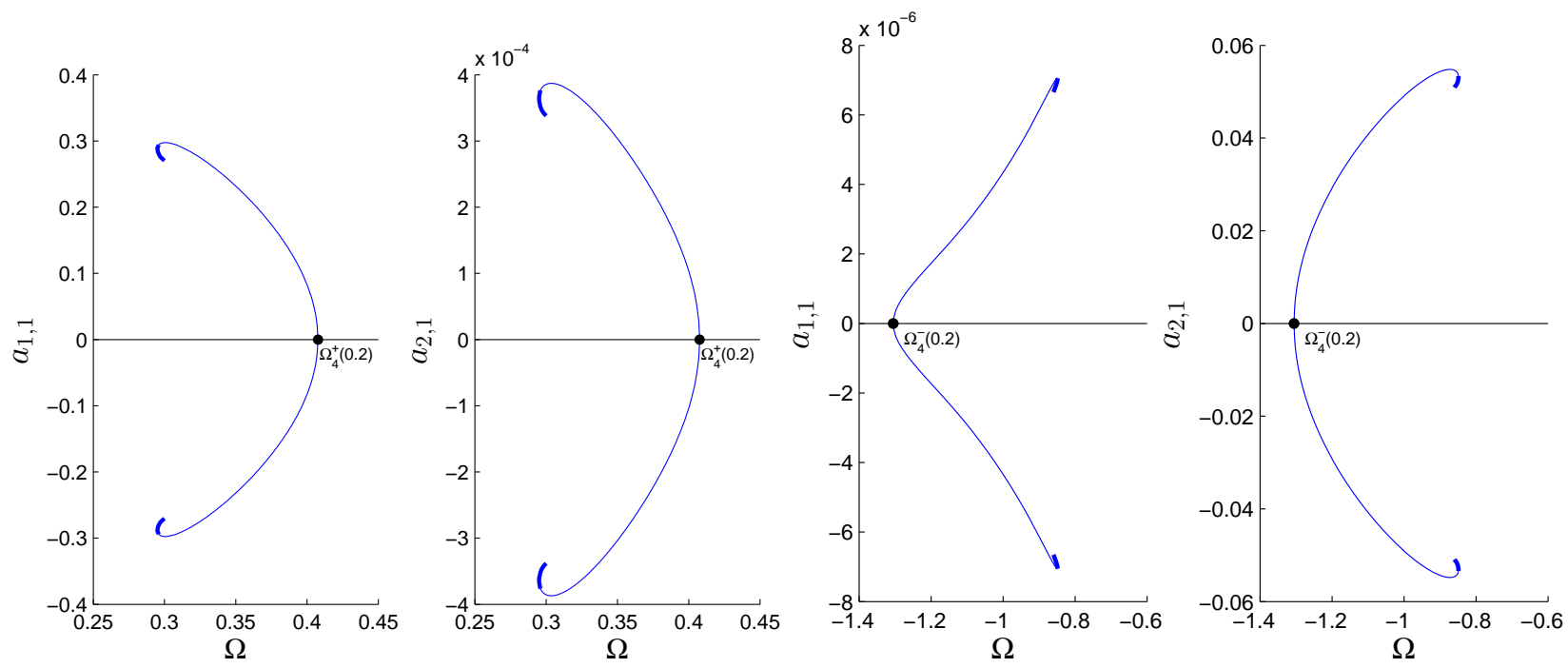

FiguRE 11. Extended bifurcation curves, for $m=4, \alpha=0.9$, and $b=0.2$. The leftmost two correspond to a bifurcation from $\Omega_{4}^{+}(0.2)$, whereas the rightmost two correspond to a bifurcation from $\Omega_{4}^{-}(0.2)$. Remember that $a_{1,1}$ and $a_{2,1}$ are such that $a_{1,1} \cdot a_{2,1}<0$.
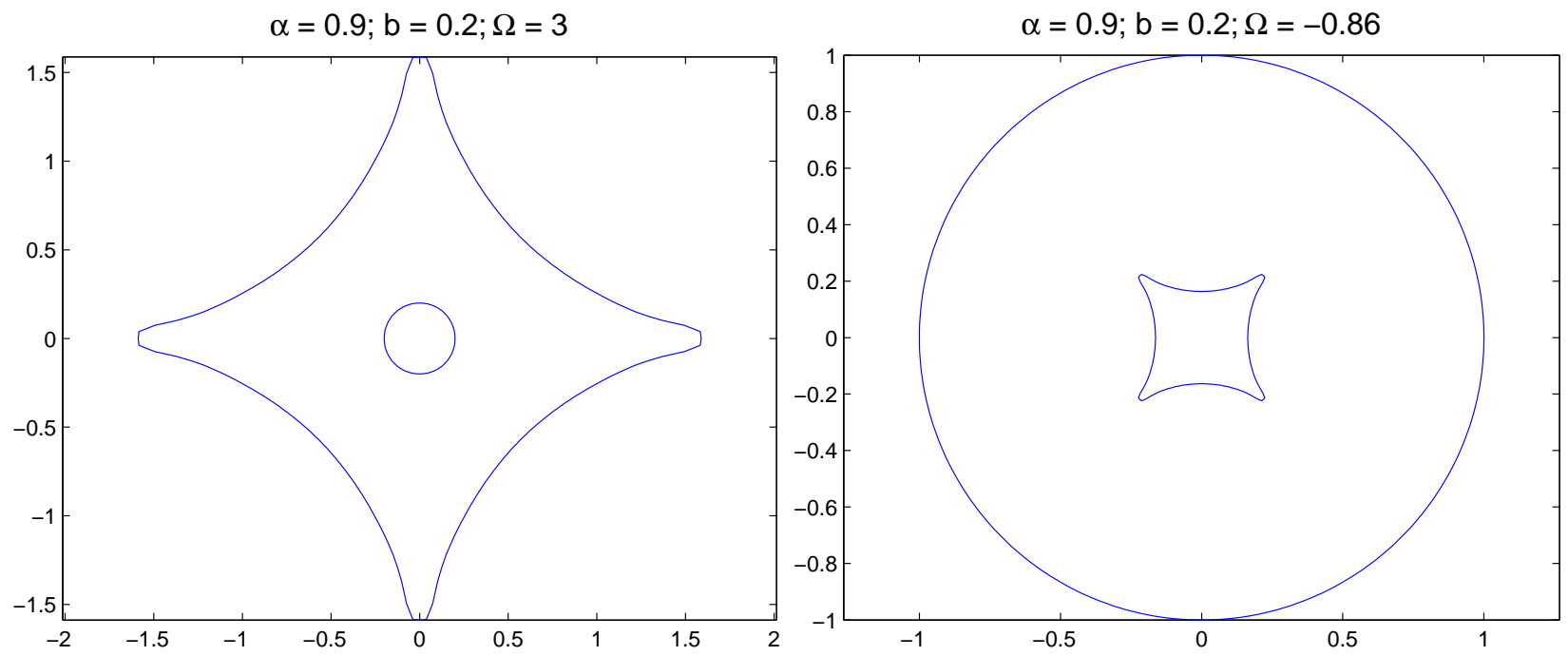

FiguRE 12. $V$-states beyond the saddle-node bifurcation points.

left-hand side of Figure 13, we plot the $V$-states corresponding to $\Omega=0.082,0.093, \ldots, 0.148$. The $V$-state corresponding to $\Omega=0.148$ (in red), and to $\Omega=0.082$ (in black), are practically circular annuli (we use no thicker stroke here, because all the $V$-states are very close to each other). On the right-hand side of Figure 13, we plot the bifurcation curves of $a_{1,1}$ and $a_{2,1}$ in (7.25), with respect to $\Omega$. As expected, the curves are closed; however, the curve corresponding to $a_{2,1}$ is much more symmetrical and reminds us of an ellipse.

Let us finish this section by mentioning the existence of stationary doubly-connected $V$ states when $\alpha>0$, i.e., $V$-states with $\Omega=0$. Like the examples with $\Omega<0$ shown above, 

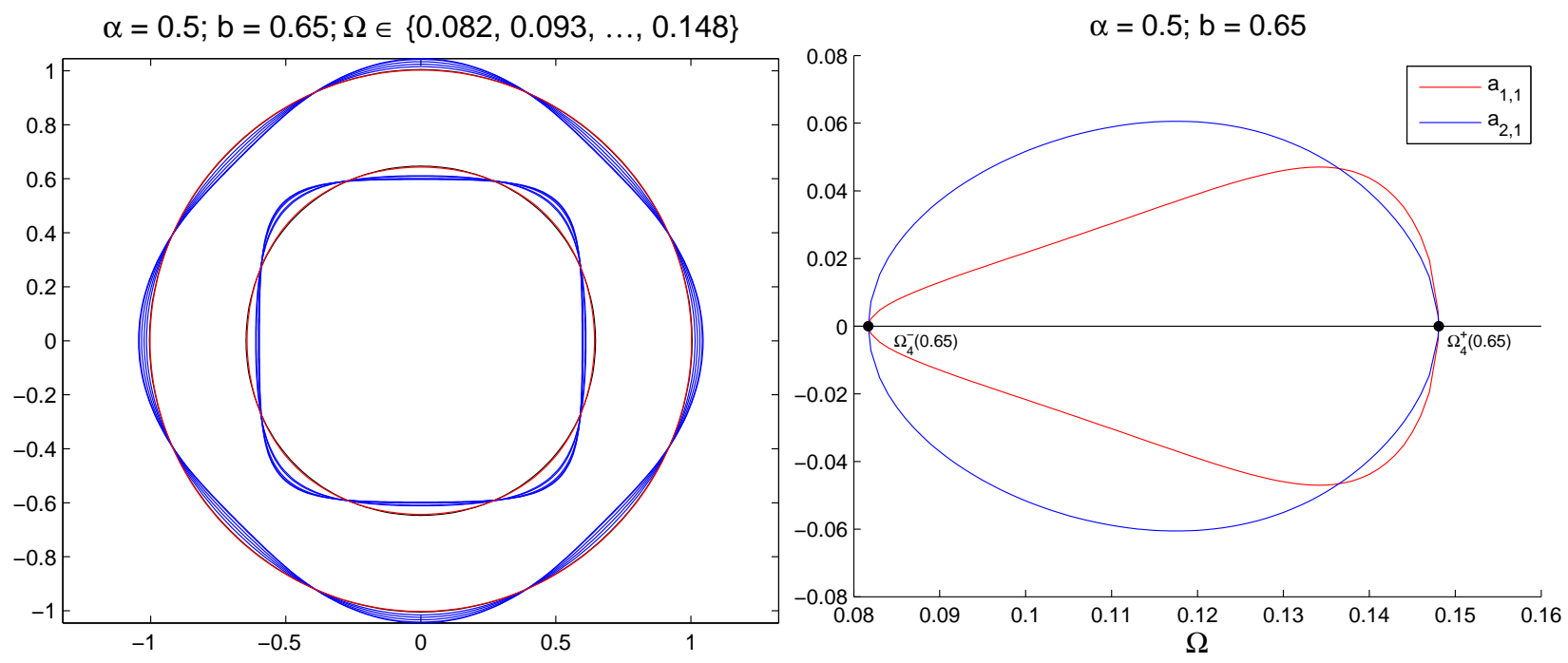

FiguRE 13. Left: 4-fold $V$-states corresponding to $\alpha=0.5, b=0.65$, for different values of $\Omega$; there are $V$-states for all $\Omega \in\left[\Omega_{m}^{-}, \Omega_{m}^{+}\right]$. Right: bifurcation curves of $a_{1,1}$ and $a_{2,1}$ in (7.25) with respect to $\Omega$. The curve corresponding to $a_{2,1}$ is much more symmetrical. Remember that $a_{1,1}$ and $a_{2,1}$ are such that $a_{1,1} \cdot a_{2,1}<0$.

they have no particularity from a numerical point of view, yet they are a completely new phenomenon with respect to the vortex patch problem. To obtain them, it is necessary to choose $m, \alpha$ and $b$, such that $\Omega_{m}^{-}<0$, but $\left|\Omega_{m}^{-}\right| \ll 1$, since we bifurcate from the annulus at $\Omega=\Omega_{m}^{-}$. We have chosen $m=4$ and $\alpha=0.5$, as in the last experiment, but with a $b$ even smaller, $b=0.5$, in such a way that $\Omega_{4}^{-}(0.5)=-0.02760 \ldots$ Figure 14 shows the corresponding stationary $V$-state.

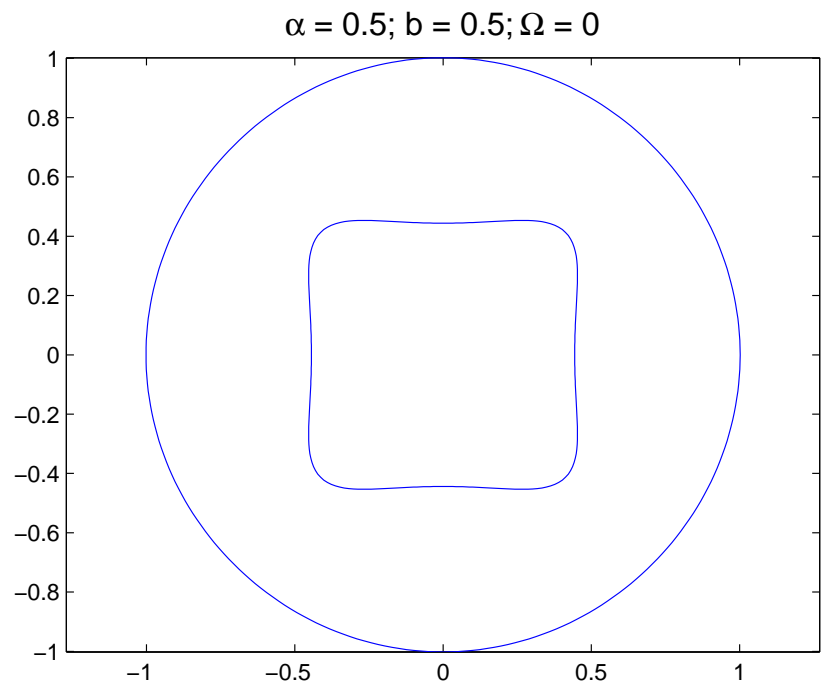

FiguRE 14. Example of a stationary $V$-state. 
Acknowledgements. Francisco de la Hoz was supported by the Spanish Ministry of Economy and Competitiveness, with the project MTM2011-24054, and by the Basque Government, with the project IT641-13. Zineb Hassainia and Taoufik Hmidi were partially supported by the ANR project Dyficolti ANR-13-BS01-0003-01.

\section{REFERENCES}

[1] M. H. P. Ambaum, B. J. Harvey, X. J. Carton. Instability of Shielded Surface Temperature Vortices. J. Atmos. Sci. 68 (2010) 964-971.

[2] M. H. P. Ambaum and B. J. Harvey, Perturbed Rankine vortices in surface quasi-geostrophic dynamics. Geophysical and Astrophysical Fluid Dynamics, 105 (4-5) (2011) 377-391.

[3] H. Aref, Integrable, chaotic, and turbulent vortex motion in two-dimensional flows. Ann. Rev. Fluid Mech., 15 (1983), 345-389.

[4] A. L. Bertozzi, P. Constantin. Global regularity for vortex patches. Comm. Math. Phys. 152 (1993), no.1, $19-28$.

[5] A. Bertozzi, A. Majda. Vorticity and Incompressible Flow. Cambridge texts in applied Mathematics, Cambridge University Press, Cambridge, (2002).

[6] J. Burbea, Motions of vortex patches, Lett. Math. Phys. 6 (1982), 1-16.

[7] A. Castro, D. Córdoba, J. Gómez-Serrano, A. Martín Zamora, Remarks on geometric properties of SQG sharp fronts and $\alpha$-patches. Discrete Contin. Dyn. Syst. 34 (2014), no. 12, 5045-5059.

[8] A. Castro, D. Córdoba, J. Gómez-Serrano. Existence and regularity of rotating global solutions for the generalized surface quasi-geostrophic equations. Preprint arXiv:1409.7040.

[9] C. Cerretelli, C.H.K. Williamson. A new family of uniform vortices related to vortex configurations before fluid merger. J. Fluid Mech. 493 (2003) 219-229.

[10] D. Chae, P. Constantin, D. Córdoba, F. Gancedo, and J. Wu, Generalized surface quasi-geostrophic equations with singular velocities. Comm. Pure Appl. Math., 65 (2012), no. 8,1037-1066.

[11] S. A. Chaplygin, On a pulsating cylindrical vortex. Translated from the 1899 Russian original by G. Krichevets, edited by D. Blackmore and with comments by V. V. Meleshko. Regul. Chaotic Dyn. 12 (2007), no. 1, 101-116.

[12] J.-Y. Chemin, Fluides Parfaits Incompressibles, , Astérisque 230 (1995); Perfect Incompressible Fluids translated by I. Gallagher and D. Iftimie, Oxford Lecture Series in Mathematics and Its Applications, Vol. 14, Clarendon Press-Oxford University Press, New York (1998).

[13] P. Constantin, A. J. Majda, and E. Tabak, Formation of strong fronts in the 2-D quasigeostrophic thermal active scalar. Nonlinearity, 7 (1994), no. 6, 1495-1533.

[14] D. Córdoba, M. A. Fontelos, A. M. Mancho and J. L. Rodrigo, Evidence of singularities for a family of contour dynamics equations. Proc. Natl. Acad. Sci. USA 102 (2005), no. 17, 5949-5952.

[15] M. G. Crandall and P.H. Rabinowitz, Bifurcation from simple eigenvalues, J. of Func. Analysis 8 (1971), 321-340.

[16] G.S. Deem and N. J. Zabusky, Vortex waves : Stationary "V-states", Interactions, Recurrence, and Breaking, Phys. Rev. Lett. 40 (1978), no. 13, 859-862.

[17] D. G. Dritschel. The nonlinear evolution of rotating configurations of uniform vorticity. J. Fluid Mech. 172 (1986), 157-182.

[18] G. R. Flierl, L. M. Polvani, Generalized Kirchhoff vortices, Phys. Fluids 29 (1986) 2376-2379.

[19] F. Gancedo, Existence for the $\alpha$-patch model and the QG sharp front in Sobolev spaces. Adv. Math., 217,6 (2008) 2569-2598.

[20] Z. Hassainia, T. Hmidi. On the V-States for the generalized quasi-geostrophic equations. Comm. Math. Phys. 337 (2015), no. 1, 321-377.

[21] I. Held, R. Pierrehumbert, S. Garner,K. Swanson, Surface quasi-geostrophic dynamics. J. Fluid Mech. 282 (1995), 1-20.

[22] T. Hmidi, On the trivial solutions for the rotating patch model. Preprint arXiv:1409.8469.

[23] T. Hmidi, F. de la Hoz, J. Mateu, J. Verdera. Doubly connected V-states for the planar Euler equations. Preprint arXiv:1409.7096. 
[24] T. Hmidi, J. Mateu, J. Verdera, Boundary Regularity of Rotating Vortex Patches. Arch. Ration. Mech. Anal. 209 (2013), no. 1, 171-208.

[25] T. Hmidi, J. Mateu, J. Verdera, On rotating doubly connected vortices. J. Differential Equations 258 (2015), no. 4, 1395-1429.

[26] M. Juckes, Quasigeostrophic dynamics of the tropopause/ J. Armos. Sci. (1994) 2756-2768.

[27] S. Kida, Motion of an elliptical vortex in a uniform shear flow. J. Phys. Soc. Japan. 50 (1981) 3517-3520.

[28] H. Kielhöfer, Bifurcation Theory: An Introduction With Applications to Partial Differential Equations, Springer ( 2011).

[29] G. Kirchhoff, Vorlesungen uber mathematische Physik (Leipzig, 1874).

[30] H. Lamb, Hydrodynamics, Dover Publications, New York, (1945).

[31] G. Lapeyre, P. Klein, Dynamics of the upper oceanic layers in terms of surface quasigeostrophic theory. J. Phys. Oceanogr. 36 (2006), 165-176.

[32] X. Liu, M. Wang, Z. Zhang. Local well-posedness and blow-up criterion of the Boussinesq equations in critical Besov spaces. Journal of Mathematical Fluid Mechanics, 12, (2010) 280-292.

[33] P. Luzzatto-Fegiz, C. H. K. Williamson. Stability of elliptical vortices from "Imperfect-Velocity-Impulse" diagrams. Theor. Comput. Fluid Dyn. 24 (2010), no. 1-4, 181-188.

[34] W. Magnus, F. Oberhettinger Formeln und satze fur die speziellen funktionen der mathematischen physik, Berlin.Gottingen.Heidelberg : Springer, 1948 .

[35] J. Neu, The dynamics of columnar vortex in an imposed strain. Phys. Fluids 27 (1984), 12397-2402.

[36] P. K. Newton, The N-Vortex Problem. Analytical Techniques, Springer, New York, (2001).

[37] E. A. II Overman. Steady-state solutions of the Euler equations in two dimensions. II. Local analysis of limiting V-states. SIAM J. Appl. Math. 46 (1986), no. 5, 765-800.

[38] Ch. Pommerenke, Boundary behaviour of conformal maps, Springer-Verlag, Berlin, 1992.

[39] E. D. Rainville. Special Functions. Macmillan, New York, (1960).

[40] J. L. Rodrigo, On the evolution of sharp fronts for the quasi-geostrophic equation, Comm. Pure Appl. Math. 58 (2005), no. 6 821-866.

[41] P. G. Saffman. Vortex dynamics. Cambridge Monographs on Mechanics and Applied Mathematics. Cambridge University Press, New York, 1992.

[42] P. G. Saffman, R. Szeto. Equilibrium shapes of a pair of equal uniform vortices. Phys. Fluids 23 (1980), no. $12,2339-2342$.

[43] S. E. Warschawski. On the higher derivatives at the boundary in conformal mapping. Trans. Amer. Math. Soc. 38 (1935), no. 2, 310-340.

[44] Watson, A Treatise on the Theory of Bessel Functions. Cambridge University Press , 1944. Trans. Amer. Math. Soc. 299 (1987), no. 2, 581-599.

[45] H.M. Wu, E.A. Overman II and N.J. Zabusky Steady-state solutions of the Euler equations in two dimensions: rotating and translating $V$-states with limiting cases I. Algorithms ans results, J. Comput. Phys. 53 (1984), 42-71.

[46] V.I. Yudovich, Non-stationnary flows of an ideal incompressible fluid. Zhurnal Vych Matematika, 3, pages 1032-106, 1963.

[47] N. Zabusky, M. H. Hughes, K.V. Roberts, Contour dynamics for the Euler equations in two dimensions. J. Comput. Phys. 30 (1979), no. 1, 96-106.

[48] Y. Zhou. Local well-posedness for the incompressible Euler equations in the critical Besov spaces. Ann. de l'Institut Fourier, 54 (2004), 3, 773-786.

Department of Applied Mathematics and Statistics and Operations Research, Faculty of Science and Technology, University of the Basque Country UPV/EHU, Barrio Sarriena S/N, 48940 LEIOA, SPAIN

E-mail address: francisco.delahoz@ehu.es

IRMaR, Université de Rennes 1, Campus de Beaulieu, 35042 Rennes cedex, France E-mail address: zineb.hassainia@univ-rennes1.fr

IRMaR, Université de Rennes 1, Campus de Beaulieu, 35042 Rennes Cedex, France E-mail address: thmidi@univ-rennes1.fr 